\title{
5 Korpora und Charakterisierung der Erscheinungsformen der Texte des Burnout-Diskurses
}

\subsection{Begründung der Korpusauswahl und Beschreibung der Diskursstränge}

\subsubsection{Einleitung in dieses Kapitel}

„Wissen über Gesundheit und Krankheit hat gesellschaftlich und diskursiv einen hohen Stellenwert und existiert als sprachlich gebundenes Wissen mit eigenem Geltungsanspruch in massenmedial kommunizierenden Gesellschaften“ (Busch 2015: 369). Schriftliche Texte der Gesundheitsberichterstattung sind in den Massenmedien allgegenwärtig, und sie können damit das Gesundheitserleben einer Gesellschaft maßgeblich beeinflussen. Die vorliegende Arbeit nimmt daher schriftliche Texte (Print- und Online-Texte) als Datengrundlage für die linguistische Diskursanalyse in den Blick.

Um die diachrone Entwicklung der diskursiven Definitionsprozesse zum Phänomen/Begriff >BURNOUT` nachzeichnen zu können, wurden schriftliche Texte aus Fachlexika, Hand- und Lehrbüchern, Fachzeitschriften, Monografien, populärwissenschaftlichen Zeitschriften, Printmedien und von Webseiten öffentlicher Akteure ausgesucht. Der Vorteil von Printtexten im Vergleich zu Texten, die nur online erscheinen, ist, dass sie überdauern und als Zeitzeugen über mehrere Jahrzehnte nachverfolgt werden können. ${ }^{212}$ Vor allem in den Jahren nach der Jahrtausendwende stiegen allerdings die im Internet veröffentlichten Texte zum Phänomen BURNOUT rapide an, sodass diese ebenfalls einen wichtigen wissens- und meinungsbildenden Faktor darstellen. Aus diesem Grund wurden auch Texte aus der deutschen Wikipedia von 2004-2019 und Texte von Webseiten („NetDoktor“ und „Onmeda“), die Informationen zu verschiedenen Krankheits- und Gesundheitsthemen veröffentlichen, für die Analyse heruntergeladen. Zudem wurden auch die Printmedien um Online-Texte desselben Mediums ergänzt (z. B. von ZEIT Online, SPIEGEL Online oder www.apotheken-umschau.de).

Das Ziel der Korpuszusammenstellung war es, aus dem „virtuellen Korpus“ (vgl. Busse/Teubert 1994: 14) eine Auswahl an konkreten Texten zu treffen, die die Heterogenität des wissenschaftlichen und gesellschaftlich-politischen Feldes

212 Mautner prägte den Satz: Gedrucktes ist: „more permanent than most web material“ (Mautner 2008: 32).

Ә Open Access. ( 2021 Theresa Schnedermann, publiziert von De Gruyter. (cc) BY-Nc-ND Dieses Werk ist lizenziert unter einer Creative Commons Namensnennung-Nicht-kommerziell-Keine Bearbeitung 4.0 International Lizenz. https://doi.org/10.1515/9783110727838-005 
zum Thema „Burnout“ sowie dessen zeitliche Entwicklung exemplarisch abbildet. Die Arbeit folgt damit bei der Korpuszusammenstellung dem Verständnis konkreter Textkorpora von Busse/Teubert (1994: 14ff.; vgl. dazu auch Warnke/ Spitzmüller (2011: 83). Um der Frage nachzugehen, wie das Phänomen BuRNouT im Diskurs aufkommt, sich verbreitet und diskursiv-definitorisch verfestigt, wurden Texte unterschiedlicher Kommunikationszusammenhänge ausgewählt, die Burnout zum Thema haben und teilweise untereinander explizite und implizite „semantische Beziehungen aufweisen“ (Busse/Teubert 1994: 14). Mit der Wahl bestimmter zeitlicher Ausschnitte (die Begründung erfolgt in Kap. 5.1.2 und 5.1.3) und Fachdomänen, insbesondere aus dem Bereich der Psychologie und Medizin (vgl. ebenfalls Kap. 5.1.2), wird eine für die Fragestellung der Arbeit multiperspektivische Auswahl an Artikeln den diskurslinguistischen Analysen zugrunde gelegt. ${ }^{213}$

Die Erstellung der Textkorpora begann semasiologisch über die Suchwörter Burnout, Ausbrennen, ausgebrannt, Ausgebranntsein in ihren verschiedenen Schreibweisen ${ }^{214}$. Diese semasiologische Suchstrategie eignet sich für das hiesige Erkenntnisinteresse gut, da die zu untersuchenden Definitionsprozesse an diesen Ausdruck rückgebunden sind. Dennoch wurden auch Texte onomasiologisch erschlossen, um etwaige Veränderungen in der terminologischen Ordnung und implizite Abgrenzungspraktiken oder semantische Kämpfe erkennen zu können. Die onomasiologische Erschließung des Phänomens geschah im Fachdiskurs über Thesauri einschlägiger Datenbanken (siehe Kap. 5.1.2) sowie durch intertextuelle Verweise oder Wiederaufnahmestrukturen in den zu Beginn analysierten Texten. Im fachexternen Korpus wurden des Weiteren Ausgaben der Zeitschrift

213 Mit dem Konzept eines multiperspektivischen konkreten Textkorpus wird der Tatsache begegnet, dass nicht alle überlieferten Texte eines Diskurses, d.h. „das Arsenal an Dokumenten, das der Analyse also prinzipiell zugänglich ist“ (Spitzmüller/Warnke 2011: 83), untersucht werden kann. Die Zusammenstellung erhebt nicht den Anspruch, den Gesamtdiskurs im Sinne eines „imaginären“ oder „,virtuellen“ Textkorpus (ebd., mit Bezug auf Busse/Teubert 1994: 14ff.) zum Phänomen BURNoUT repräsentativ abzubilden. Dazu würden neben 69.900.000 Ergebnissen in der Suchmaschine Google zum Suchwort Burnout (Stand: 17.11.2019) schließlich auch private Gespräche über dieses Thema und viele weitere Texte gehören. Leitidee bei der Zusammenstellung der Texte war daher die Multiperspektivität in horizontaler (verschiedene Fachbereiche, Akteure), vertikaler (Fach-, Vermittlungs- und alltagsnahe Texte) und diachroner Hinsicht. Die Arbeit untersucht diskursive Praktiken des Definierens in geschriebenen Texten. Texte gesprochener Sprache wurden daher in dieser Arbeit ausgeklammert. Ein Vergleich von Definierenspraktiken in geschriebenen und gesprochenen Texten ist jedoch ein interessantes Forschungsdesiderat für Anschlussstudien.

214 Weitere Schreibweisen im Englischen und Deutschen sind: Burn-out, Burn-Out, burned out, burnt out, Burnout-Syndrom, Burn-out-Syndrom. 
„Apotheken Umschau“ und „Psychologie Heute“ seit den 1970er und 1980er Jahren gesichtet (zur genauen Recherche-Aufschlüsselung siehe Kap. 5.1.3).

Das Textkorpus gliedert sich in drei Teilkorpora: Es besteht erstens aus Einträgen aus Neologismenwörterbüchern, aus allgemeinen einsprachigen Wörterbüchern vor und nach 1970 und Treffern zu den Suchwörtern ausbrennen und ausgebrannt in historischen Textkorpora (DTA 1473-1927 und DWDS-Kernkorpus 1900-1999), um die Entwicklung der Verwendung der Verben ausbrennen sowie to burn out und der daraus abgeleiteten Nomen Ausbrennen und Ausgebranntsein sowie Burnout/Burn-out diachron nachzuvollziehen (= Teil 1a und 1b). Das zweite Teilkorpus (= Teil 2a und 2b) bilden innerfachliche und interfachliche (Vermittlungs-)Texte, d. h. dieses Teilkorpus besteht einerseits aus Texten, die von Fachleuten eines spezifischen Fachbereichs wie z. B. der Arbeits- und Organisationspsychologie für Fachleute desselben Bereichs geschrieben worden sind. Andererseits enthält es auch interdisziplinäre Vermittlungstexte, wenn beispielsweise ein/e Psychiater/in einen Informationsbeitrag über >Burnout` in der Zeitschrift „Der Internist“ veröffentlicht oder Fachleute Wissen über einen Gegenstand in Handbüchern oder Fachlexika für die fachliche Lehr-Lern-Situation aufbereiten. Der dritte Teil des Korpus (= Teil 3) enthält sogenannte populärwissenschaftliche, jedem öffentlich ${ }^{215}$ zugängliche Vermittlungstexte, in denen „ein Wissensbestand und das zugehörige terminologische Feld zusammenhängend und explizit didaktisiert für eine Zielgruppe dargeboten“ (Jung 1999: 193) werden und Texte des „Typus der öffentlichen Vermittlung im (politischen) Mediendiskurs“ (ebd.: 196), in denen der Fokus nicht nur auf der explizit didaktisierten Wissensvermittlung, sondern auch auf gesellschaftlichen und politischen Ereignissen liegt. Es werden (Vermittlungs-)texte sowohl aus populärwissenschaftlichen Zeitschriften als auch aus klassischen Print-/Onlinemedien und Onlineplattformen (Wikipedia, NetDoktor, Onmeda) untersucht. Folgendes Schaubild (Abbildung 5) bietet eine Übersicht über die untersuchten Teilkorpora. In den folgenden Kapiteln wird die Zusammenstellung der Teilkorpora ausführlich dargelegt.

215 Mit dem Ausdruck öffentlich soll verdeutlicht werden, dass die Texte, die in Massenmedien oder Magazinen mit hoher Verkaufsauflage erscheinen, eine breitere und heterogenere Öffentlichkeit ansprechen als die fachinternen Texte, die sich an Fachleute des eigenen Spezial- oder übergeordneten Fachs bzw. Fachleute anderer Disziplinen richten. Des Weiteren soll an dieser Stelle darauf hingewiesen werden, dass Fachtexte zwar öffentlich erscheinen, dass der Zugang zu ihnen aber nicht nur durch ihren Fachlichkeitsgrad, sondern auch durch die Organisation ihrer Verbreitung (teurer Anschaffungspreis, meist nur auffindbar über Kenntnisse in bibliografischen Recherchepraktiken, kleinere Auflage) erschwert wird. Foucault fasst solche Prozeduren, die den Subjekten den Zugang zum Diskurs erleichtern oder erschweren, unter der Bezeichnung der „Verknappung der sprechenden Subjekte“ zusammen (Foucault [1970] 1993: 26ff.). 


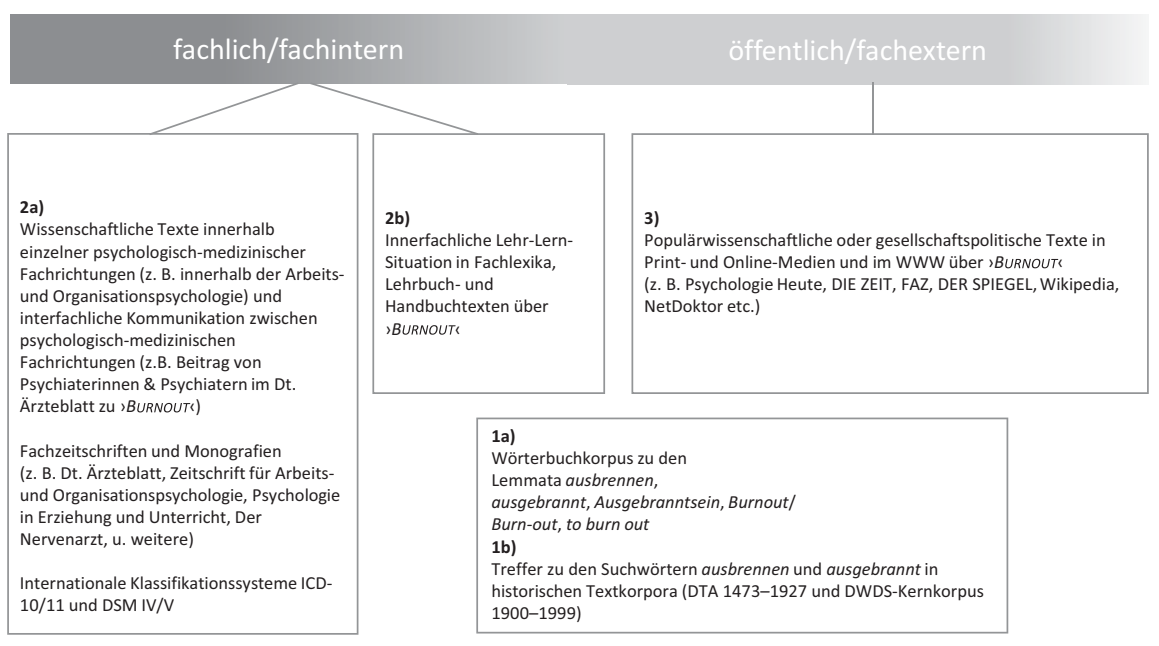

Abb. 5: Übersicht zu den Teilkorpora des Untersuchungskorpus.

\subsubsection{Wörterbuch- und Datenbankkorpus zu den Lesarten des Verbs ausbrennen/to burn out vor dem 20./21. Jahrhundert}

Das Wörterbuch- und Datenbankenkorpus besteht einerseits aus allgemeinen deutschen und englischen Wörterbüchern, Neologismenwörterbüchern und zweisprachigen Wörterbüchern (englisch-deutsch), darunter auch Wörterbücher des 19. Jahrhunderts, und andererseits aus historischen Textkorpora (DTA 1473-1927 und DWDS-Kernkorpus 1900-1999) ${ }^{216}$ und Suchanfragen im Deutschen

216 Gesucht wurde erstens im DTA = Deutsches Textarchiv (1473-1927), dessen Textauswahl unter folgender Adresse nachgelesen werden kann: http://www.deutschestextarchiv.de/doku/textauswahl; und zweitens im Kernkorpus des Digitalen Wörterbuchs der deutschen Sprache=DWDSKernkorpus 1900-1999. Dessen Textauswahl ist unter folgender Adresse dokumentiert: https://www. dwds.de/d/k-referenz\#kern. DieSuche nach ausbrennen ergab im DTA in allen „Textklassen“ 194 Treffer (https://www.dwds.de/r/?q=ausbrennen\&corpus=dta\&date-start=1473\&date-end=1927\&genre= Belletristik\&genre=Wissenschaft\&genre $=$ Gebrauchsliteratur\&genre $=$ Zeitung\&format $=$ full\&sort $=-$ date_asc\&limit=100, letzter Stand: 7.12.2018) und nach ausgebrannt 541 Treffer (https://www.dwds. de/r?q=ausgebrannt\&corpus=dta\&date-start=1473\&date-end=1927\&genre=Belletristik\&genre=Wissenschaft\&genre=Gebrauchsliteratur\&genre=Zeitung\&format=full\&sort=date_asc\&limit=100, letzter Stand: 7.12.2018). Die Suche nach ausbrennen im DWDS-Kernkorpus 1900-1999 ergab 50 Treffer, von denen aus urheberrechtlichen Gründen aber nur 38 Treffer angezeigt werden (https:// www.dwds.de/r?q=ausbrennen\&corpus=kern\&date-start=1900\&date-end=1999\&genre=Belletristik \&genre=Wissenschaft\&genre=Gebrauchsliteratur\&genre=Zeitung\&format=full\&sort=date_asc\& limit=10, letzter Stand: 7.12.2018). Die Suche nach ausgebrannt im DWDS-Kernkorpus 1900-1999 
Referenzkorpus des Leibniz-Instituts für Deutsche Sprache (DEREKo) ${ }^{217} \mathrm{zu}$ den Suchformen Ausgebranntsein* und den substantivierten Formen das Ausbrennen/des Ausbrennens/dem Ausbrennen. ${ }^{218}$ Mithilfe der Wörterbucheinträge und historischen Textkorpora wird der Frage nachgegangen, ob die Ausdrücke (to) burn out / burnt out und ausbrennen/ausgebrannt schon vor dem Beginn des psychologisch-medizinischen Diskurses in ähnlich ,übertragener‘ Bedeutung im allgemeinen Sprachgebrauch üblich waren (siehe Kap. 6.1.2). Des Weiteren können diese Wörterbücher und insbesondere auch die Neologismenwörterbücher des Englischen und Deutschen neben den Medientexten Indizien dafür liefern, wann die Verwendung der Substantive Ausgebranntsein, Burnout oder Burnout-Syndrom einsetzte und $\mathrm{zu}$ welchem Zeitpunkt die Verwendung dieser Bezeichnungen für ein spezifisches psychisches Phänomen als 'neu' empfunden wurde.

Folgende Wörterbücher werden untersucht (siehe auch im QV unter 8.1.1):

\section{Allgemeine einsprachige Wörterbücher der deutschen Sprache}

- Campe, Joachim Heinrich (1807): Wörterbuch der Deutschen Sprache. 1. Band. Braunschweig: Schulbuchhandlung. ${ }^{219}$

- Adelung, Johann Christoph ([21793] 1970): Grammatisch-kritisches Wörterbuch der Hochdeutschen Mundart mit beständiger Vergleichung der übrigen Mundarten, besonders aber der oberdeutschen. 1. Theil, von A-E. Zweyte, vermehrte und verbesserte Ausgabe. Leipzig: Breitkopf. Reprografischer Nachdruck mit einer Einführung von Helmut Henne. Hildesheim: Olms. ${ }^{220}$

- Heinsius, Theodor (1828-1830): Vollständiges Wörterbuch der deutschen Sprache mit Bezeichnung der Aussprache und Betonung für die Geschäftsund Lesewelt. 1. Teilband. Wien: Schade. ${ }^{221}$

\footnotetext{
ergab 266 Treffer, von denen 188 Treffer angezeigt werden (https://www.dwds.de/r?q=ausgebrannt\&corpus=kern\&date-start=1900\&date-end=1999\&genre=Belletristik\&genre=Wissenschaft\& genre=Gebrauchsliteratur\&genre=Zeitung\&format=full\&sort=date_asc\&limit=10, Stand 7.12.2018).

217 Zur Zusammenstellung des Deutschen Referenzkorpus siehe: https://www.ids-mannheim. de/digspra/kl/projekte/korpora/. Die Recherche erfolgt über das Corpus Search, Management and Analysis System Cosmas 2: https://www.ids-mannheim.de/cosmas2/ (Stand: 30.09.2018).

218 Die Begründung für diese Auswahl der Suchwörter in DEREKo erfolgt in Kap. 6.1.2, S. 226.

219 Teilweise online abrufbar unter: http://reader.digitale-sammlungen.de/resolve/display/ bsb10523279.html (zuletzt eingesehen am 12.12.2019).

220 Online abrufbar unter: http://woerterbuchnetz.de/cgi-bin/WBNetz/wbgui_py?sigle=Adelung \&mode=Vernetzung\&hitlist=\&patternlist=\&lemid=DA00001 (zuletzt eingesehen am 12.12.2019).

221 Online abrufbar unter: https://babel.hathitrust.org/cgi/pt?id=hvd.hn4vjr;view=1up;seq=7 (zuletzt eingesehen am 12.12.2019).
} 
- Heyse, Johann Christian August (1833): Handwörterbuch der deutschen Sprache. Band 1, A-K. Nachdruck von Georg Olms 1968. Hildesheim: Olms.

- ${ }^{1}$ DWB (1854-1960) = Deutsches Wörterbuch von Jacob Grimm und Wilhelm Grimm. Leipzig: Hirzel. ${ }^{222}$

- $\quad{ }^{2}$ DWB = Deutsches Wörterbuch von Jacob Grimm und Wilhelm Grimm. Neubearbeitung, hg. von der Berlin-Brandenburgischen Akademie der Wissenschaften (vormals Akademie der Wissenschaften der DDR) und der Akademie der Wissenschaften zu Göttingen, Band 1ff. Leipzig: Hirzel $1960 \mathrm{ff}$.

- Duden (1983; ${ }^{2}$ 1989; ${ }^{3}$ 1996; ${ }^{4}$ 2001): Deutsches Universalwörterbuch. Mannheim u. a.: Bibliographisches Institut/Dudenverlag.

- Wahrig, Gerhard (1966): Das große deutsche Wörterbuch. Gütersloh: Bertelsmann.//Wahrig, Gerhard et al. (1977; 1980; 1986/1987; 1994; 2000): Deutsches Wörterbuch. Gütersloh u. a.: Bertelsmann (in anderen Ausgaben Wiesbaden: Brockhaus und München: Mosaik, siehe im QV unter 8.1.1).

\section{Allgemeine einsprachige Wörterbücher der englischen Sprache}

- Oxford English Dictionary Online (21989, 1992, 1997, 2001-2019): OnlineVersion des OED mit den vollständigen Daten der 2. Ausgabe von 1989 inklusive der Additions von 1992 und 1997 und vierteljährlicher Updates. ${ }^{223}$

- Cambridge International Dictionary of English (1995). Cambridge: Cambridge University Press.

- Merriam-Webster's Collegiate Dictionary (1975; ${ }^{10} 1993 ;{ }^{11} 2003 ;{ }^{11} 2008$ ): America's Best-Selling Dictionary; New Ways to Find the Words You Need Today. Springfield, Mass.: Merriam-Webster.

\section{Neologismenwörterbücher der deutschen und englischen Sprache}

- Berg, Paul C. (1953): A Dictionary of New Words in English. London: George Allen \& Unwin Ltd.

- Reifer, Mary (1955): Dictionary of New Words. New York: Philosophical Library.

- Barnhart, Clarence L./Steinmetz, Sol/Barnhart, Robert K. (1973; ²1980; $\left.{ }^{3} 1990\right)$ : The Barnhart Dictionary of New English 1963-1972. Bronxville, N. Y./ Berlin u. a.: Barnhart/Langenscheidt.

- Tulloch, Sara (1992): The Oxford Dictionary of New Words. A popular guide to words in the news. Oxford/New York: Oxford University Press.

- Green, Jonathan (1991): New Words - A dictionary of Neologism since 1960. London: Bloomsbury.

222 Online abrufbar unter: http://woerterbuchnetz.de/DWB/; zuletzt eingesehen am 12.12.2019. 223 Online-Ressource: http://www.oed.com/ (zuletzt eingesehen am 26.11.2019). 
- Herberg, Dieter/Kinne, Michael/Steffens, Doris (2004): Neuer Wortschatz. Neologismen der 90er Jahre im Deutschen. Berlin: de Gruyter.

- Quastoff, Uwe (2007): Deutsches Neologismenwörterbuch: neue Wörter und Wortbedeutungen in der Gegenwartssprache. Berlin u. a.: de Gruyter.

\section{Fremdwörter- und etymologische Wörterbücher}

- Duden $\left({ }^{4} 1982 ;{ }^{5} 1990 ;{ }^{6} 1997 ;{ }^{8} 2005 ;{ }^{10} 2010\right)$ : Fremdwörterbuch. Mannheim u. a.: Bibliographisches Institut/Dudenverlag.

- Duden (52014): Das Herkunftswörterbuch: Etymologie der deutschen Sprache. Redaktionelle Bearbeitung Jörg Riecke, Band 7. Berlin/Mannheim/Zürich: Dudenverlag.

Im fachlichen Korpus befinden sich ebenfalls Fachlexika und Enzyklopädien. Die allgemeinen einsprachigen Wörterbücher allerdings wurden mit einem erweiterten Untersuchungsinteresse zusammengestellt. Denn es geht hier neben der Frage, wie in den Wörterbüchern der jüngeren Zeit Burnout definiert wird, darum herauszufinden, welche Lesarten und metaphorischen Verwendungsweisen im allgemeinen Sprachgebrauch für ähnliche Kontexte schon üblich waren (vgl. Kap. 6.1.2 in dieser Arbeit).

\subsubsection{Korpuserstellung des fachlichen Diskursstrangs seit 1975}

Die Erstellung des Fachdiskurses beruht erstens auf Vorrecherche in Datenbanken (PSYNDEX ${ }^{224}$, Pubpsych ${ }^{225}$ und PsycINFO ${ }^{226}$ ) mit den Suchwörtern „burnout

224 Informationen zur Datenbank aus der Beschreibung der Universitätsbibliothek Heidelberg: „Bibliographische Datenbank zu psychologischer Literatur und Testverfahren aus den deutschsprachigen Ländern sowie psychologisch relevanten audiovisuellen Medien aus allen Gebieten der Psychologie einschließlich psychologisch relevanter Aspekte aus Nachbardisziplinen wie Psychiatrie, Medizin, Erziehungswissenschaft, Soziologie, Sportwissenschaft, Linguistik, Betriebswirtschaft, Kriminologie.“

225 Frei abrufbar unter: https://pubpsych.zpid.de/pubpsych/ (zuletzt eingesehen am 17.11.2019). 226 Informationen zur Datenbank aus der Beschreibung der Universitätsbibliothek Heidelberg: „PsycINFO verzeichnet Zeitschriftenaufsätze, Bücher, Buchkapitel, Buchbesprechungen, Forschungsberichte, Fallstudien etc. zur Psychologie und verwandten Gebieten wie Psychiatrie, Soziologie, Erziehungswissenschaften, Anthropologie, Pharmakologie, Physiologie, Kriminologie und Linguistik, soweit sie für die Psychologie von Interesse sind. Ausgewertet werden circa 2.000 Zeitschriften. Insgesamt bietet PsycInfo circa 60.000 Neueintragungen pro Jahr mit wöchentlichen Updates an. PsycINFO wird von der American Psychological Association (APA) produziert.“ Zum Berichtszeitraum werden folgende Angaben gemacht: „Zeitschriftenaufsätze ab 1887; Bücher/Buchkapitel ab 1987“. 
ODER burn-out ODER ausgebrannt“ und einer Thesaurus-Auswertung weiterer einschlägiger Suchbegriffe. Um einen Eindruck davon zu erhalten, welche Phänomene/Konzepte von Fachleuten als themenverwandt $z u$ >BURNOUT< angesehen werden, wurden zu Beginn alle akademischen Zeitschriftenartikel in der Datenbank PSYNDEX gesucht, die die Suchwörter burnout oder burn-out oder ausgebrannt im Titel tragen. Bei diesen 392 Artikeln von 1982-2016 wurde ausgewertet, welche Schlagwörter die Fachautorinnen und -autoren zu ihren Beiträgen über >BURNOUT< aus einer Thesaurus-Auswahl der Datenbank Psyndex vergeben haben. ${ }^{227}$ Dabei erwiesen sich vor allem occupational stress/beruflicher Stress bei 334, prevention/Prävention bei 82, stress reactions/Stressreaktionen bei 79 und working conditions/Arbeitsbedingungen bei 72 Treffern als sachverwandte Schlagwörter. Des Weiteren kann man bei diesen Schlagwörtern sehen, welche Berufe von Beginn an untersucht wurden: Lehrer erscheinen bei 41 Texten und Lehrermerkmale bei 19 Texten als sachverwandte Schlagwörter, Krankenpflegepersonal bei 29, Ärzte bei 17, Führungskräfte bei 10 und Altenpflege bei 9 Texten.

Parallel wurden verschiedene psychologische, soziologische und medizinische Fachlexika, Handbücher und Lehrwerke konsultiert und jeweils Artikel zum Thema „Burnout“ extrahiert und in das Korpus aufgenommen (siehe den Abschnitt 8.1.2 mit 84 Titeln in der Quellenliste im Anhang). Anhand dieser Anfangsrecherchen in Lexika und Handbüchern, aber auch durch die induktive Durchsicht der Zeitschriftenartikel aus der Datenbank PSYNDEX konnte ein Profil erstellt werden, welche Fächer der Psychologie und Medizin sich vorwiegend mit dem Phänomen/Begriff >BURNOUT beschäftigen. Ausgehend von diesen Recherchen erwiesen sich vor allem die Arbeits- und Organisationspsychologie sowie Pädagogische Psychologie und kleinere Fächer wie die Medizinische Psychologie und Soziologie, Arbeitsmedizin und verschiedene psychotherapeutische Schulen (z. B. die systemische oder Verhaltenstherapie) und seit den 2000er Jahren auch die Gesundheitspsychologie und Biopsychologie als einschlägig. Die klinische Psychologie/Psychiatrie wird ebenfalls in Lexika als einschlägiges Fach genannt (vgl. Dorsch ${ }^{16} 2013$ : $312 \mathrm{im} \mathrm{QV} \mathrm{8.1.2).}$

Auf dieser Grundlage wurde nach einschlägigen Zeitschriften dieser Fachbereiche gesucht. Das „Deutsche Ärzteblatt“ wurde als auflagenstärkstes Publikationsorgan in Deutschland, das verschiedene medizinische Fachbereiche übergreifend behandelt und auch fachpolitische Entwicklungen abbildet, und „Der Nervenarzt“ als eine der auflagenstärksten deutschsprachigen Fachzeitschriften im Bereich der Psychiatrie, Psychotherapie und Psychosomatik aufgenommen. Des

227 Diese Treffer wurden chronologisch aufsteigend im Hinblick auf ihre gemeinsame Verschlagwortung über den Filter „Subject“ ausgewertet (die letzte Recherche in Psyndex erfolgte im Mai 2017). 
Weiteren wurden als Beispiele für eine interfachliche Vermittlungssituation Beiträge aus fachlich weiter entfernten Zeitschriften aufgenommen, wenn Fachleute, die $\mathrm{zu}$ >BURNOUT forschen oder in einem dazu einschlägigen Bereich praktisch tätig sind, Fachleuten anderer Unterdisziplinen (z. B. der inneren oder der Allgemeinmedizin) Ergebnisse einer Studie oder den gegenwärtigen Forschungsstand zu diesem Thema berichten. ${ }^{228}$ Zudem wurden Expertinnen und Experten des psychologischen Seminars Heidelberg und Universitätsklinikums Heidelberg danach befragt, welche deutschsprachigen Zeitschriften Renommee in den einzelnen Fachbereichen besitzen. ${ }^{229}$ Es wurden überwiegend deutschsprachige Texte in das Fachtextkorpus aufgenommen, da die Anwendungsfächer (wie z. B. die Arbeitsund Organisationspsychologie oder Pädagogische Psychologie) nach einem vom Leibniz-Zentrum für Psychologische Information und Dokumentation (ZPID) veröffentlichten Monitor aus dem Jahr 2016 die „niedrigsten englischsprachigen Anteile“ aufweisen (zum sog. „Sprachenstreit“ siehe Kapitel 5.2, S. 171).

Ausgehend von diesen Recherchen erfolgte eine besonders detaillierte Recherche in den folgenden Fachzeitschriften ${ }^{230}$ (siehe im Quellenverzeichnis unter 8.1.4): „Deutsches Ärzteblatt“ (Dt. Ärztebl., 63 Texte) ${ }^{231}$, „Deutsche medizinische Wochenschrift“ (DMW, 8 Texte), „Der Nervenarzt“(13 Texte), „Psychologie in Erziehung und Unterricht“ (PiEU, 20 Texte), „Psychotherapie im Dialog “ (PiD, 16 Texte), „Psychotherapie, Psychosomatik, Medizinische Psychologie“ (PPmP, 19 Texte), „Zeitschrift für Arbeits- und Organisationspsychologie“ (ZfAO, 18 Texte).

Texte aus weiteren deutschsprachigen Fachzeitschriften wie z. B. „Zeitschrift für Arbeitswissenschaft“ oder „Zeitschrift für klinische Psychologie“ und internationalen Zeitschriften wie z. B. „Work and stress“232 oder „Anxiety

228 Wie zum Beispiel der Beitrag von dem Psychiater H.P. Kapfhammer in der Zeitschrift „Der Internist“ (11/2012: 1276-1288, im QV unter 8.1.5) oder der Beitrag des Autorenteams der Klinik und Poliklinik für Psychiatrie und Psychotherapie der TU München in der Deutschen Medizinischen Wochenschrift (2014/139: 2587-2596, = Kissling/Mendel/Förstl 2014, im QV unter 8.1.4). 229 Ich danke Prof. Dr. Ursula Christmann, Prof. Dr. Thomas Fuchs, Prof. Dr. Joachim Funke, apl. Prof. Dr. Alexandra Michel und Prof. Dr. Birgit Spinath.

230 Über Recherche in den Archiven der Zeitschriften mit den Suchwörtern burnout/burn-out/ ausgebrannt/ausbr* sowie teilweise ergänzt durch occupational stress/beruflicher stress und stress reactions oder manuelle Durchsicht.

231 Beim Ärzteblatt wurden auch Texte der Rubrik „Nachrichten“ und „Leserbriefe“ mit aufgenommen, um das Diskussionspotential rund um das Thema „Burnout“ zu erfassen und im Korpus repräsentiert zu haben.

232 Diese Zeitschrift besitzt für Zeitschriften im psychosozialen Bereich einen hohen Impactfaktor 2.467 (von 2015) (siehe dazu http://www.tandfonline.com/toc/twst20/current, zuletzt eingesehen am 15.5.2017) . 
Stress and Coping“233 und einige Monografien und Beiträge in Sammelbänden wurden des Weiteren in das fachliche Korpus aufgenommen, wenn sie beispielsweise ein eigenes Messinstrument oder eine eigene Definition entwickelt haben oder im weiteren fachlichen, aber auch öffentlichen Diskurs häufig bzw. an exponierter ${ }^{234}$ Stelle zitiert werden sowie Texte aus dem Bereich der Bio-, Gesundheits- und Sportpsychologie. Auf gleiche Weise wurde auch mit englischsprachigen Artikeln insbesondere der Anfangsphase verfahren. Es wurden die Beiträge aufgenommen, die häufig bzw. an exponierter Stelle im deutschsprachigen Diskurs zitiert werden oder deren Forschungsdesign, konzeptionelle Prägung oder Messinstrument wiederholt aufgegriffen werden. ${ }^{235}$ Die in diesem Abschnitt beschriebenen Texte sind im Quellenverzeichnis im Abschnitt 8.1.5 einsehbar (insgesamt 77 Texte).

Schließlich wurden noch die internationalen Diagnose- und Klassifikationssysteme „Internationale statistische Klassifikation der Krankheiten und verwandter Gesundheitsprobleme“ (in den Versionen ICD-10 und ICD-11) und das

233 Diese Zeitschrift besitzt einen Impactfaktor von 1.82 (von 2015/2016), https://www.researchgate.net/journal/1477-2205_Anxiety_stress_and_coping, (zuletzt eingesehen am 15.5.2017).

234 Eine exponierte Stelle im Diskurs stellt beispielsweise die Zitierung in der Einleitung der ersten deutschsprachigen Bibliografie zum Thema „Burnout“ (Kleiber/Enzmann 1990) oder die Erwähnung in einem Übersichtsartikel eines Handbuchs oder eines Reviews im Dt. Ärzteblatt oder in einem HTA-Bericht dar. HTA steht für „Health Technology Assessment“. Diese HTA-Berichte werden vom „Deutschen Institut für Medizinische Dokumentation und Information“ (DIMDI), einer nachgeordneten Behörde des Bundesministeriums für Gesundheit, herausgegeben. Zum Beispiel der HTA-Bericht Nr. 105 zur „Differentialdiagnostik des Burnout-Syndroms (= Korczak/ Kister/Huber 2010), der wiederum im viel zitierten Beitrag „Modediagnose Burnout“ im Ärzteblatt 46/2011:781-787 methodisch zugrunde gelegt wird. Informationen zu HTA-Berichten unter https://www.dimdi.de/dynamic/de/weitere-fachdienste/health-technology-assessment/ (zuletzt eingesehen am 3.7.2021).

235 Diese Zweisprachigkeit des Korpus wird in der folgenden Analyse nicht tiefergehend problematisiert. Damit sei nicht gesagt, dass es keine Unterschiede zwischen dem englischsprachigen und deutschsprachigen Burnout-Diskurs bzw. einzelsprachige Perspektivierungen gäbe. Diese könnten in einer kontrastiven Analyse mit einem zweisprachig-ausgewogenen Korpus auf verschiedenen sprachlichen Ebenen untersucht werden. Im Fokus dieser Analyse steht der deutschsprachige Diskurs und wie dieser die englischsprachigen Lehnwörter in den fachlichen und fachexternen Diskursstrang integriert. Die engen Beziehungen zwischen der englischen und deutschen Fachsprache der Psychologie und Psychotherapie, ähnliche metaphorische Verwendungsweisen der Ausdrücke (to) burn(out) und (aus)brennen sowie die Internationalisierung der naturwissenschaftlich ausgerichteten Fachkommunikation und der Bezug auf international anerkannte Diagnose- und Klassifikationssysteme (ICD, DSM) tragen dazu bei, dass der sprachliche Übergang relativ ,geräuschlos` erscheint. Diese Zusammenhänge werden in den Kapiteln 5.2, 5.3.3.2 und 6.1.2 skizziert. 
„Diagnostische und Statistische Manual Psychischer Störungen“ (in den Versionen DSM-4 und DSM-5) in das Fachkorpus integriert (siehe im QV unter 8.1.3).

Das fachliche Korpus wurde auf diese Weise zusammengestellt, um möglichst viele verschiedene Forschungsperspektiven auf das Burnout-Phänomen aufzunehmen. Denn nur auf diese Weise ist die Möglichkeit für verschiedene Arten, wie das Phänomen/der Begriff definiert wird, gegeben.

Im Quellenverzeichnis sind alle Texte zuerst aus den Fachlexika, Hand- und Lehrbüchern (QV 8.1.2), dann aus den Klassifikationssystemen (QV 8.1.3), den einzelnen Fachzeitschriften (QV 8.1.4) und schließlich aus weiteren Fachzeitschriften und Monografien und Sammelbänden (QV 8.1.5) jeweils alphabetisch aufgelistet. Zitate aus diesen Quellen werden (außer bei Auszählungen wie z. B. bei der Keywordanalyse in Kapitel 6.2.4.1 oder bei gesammelten Belegangaben zu sprachlichen Mitteln des Definierens) mit Autorenkennung im Fließtext oder in Fußnoten angegeben.

\subsubsection{Korpuserstellung des fachexternen Diskursstrangs seit 1975}

Die Erstellung des fachexternen Diskursstrangs beginnt mit der Durchsicht der Zeitschriften „Psychologie Heute“ $(\mathrm{PH})^{236}$ und „Apotheken Umschau“ $(\mathrm{AU})^{237}$ seit 1975. Letztere wurde bis 1990 besonders in den Jahren 1975, 1980 und 1985, ab 1990 jährlich und in den 2000er und 2010er Jahren in ausgewählten Jahrgängen $^{238}$ registriert. Durch diese Durchsicht konnten auch Texte, die sachverwandte

236 Im zweiten Quartal 2019 hat „Psychologie Heute“ (PH) eine Druckauflage von 124.137, wie die Zeitung „nach geprüften Zahlen der ,Informationsgemeinschaft zur Feststellung der Verbreitung von Werbeträgern e. V. (IVW)' auf ihrer Webseite angibt: https://www.psychologie-heute. de/service/mediadaten.html (zuletzt eingesehen am 23.12.2019).

237 Die „Apotheken Umschau“ (AU) ist eines der auflagenstärksten Magazine im Themenbereich Krankheit und Gesundheit in Deutschland. Die verbreitete Auflage (Ausgabe A und B) betrug nach der IVW 2019 im dritten Quartal rund 8.84 Millionen Exemplare. Einsehbar unter: https:// de.statista.com/statistik/daten/studie/797830/umfrage/verbreitete-auflage-der-apothekenumschau/ (zuletzt eingesehen am 23.12.2019).

238 Und zwar in den Jahren 2000, 2005, 2010 bis 2015 und im Jahr 2017 von Januar bis Mai 2017. Die Auswahl orientiert sich an den quantitativen Auswertungen zum Aufkommen des Ausdrucks Burnout im Deutschen Referenzkorpus (DEREKo) des Leibniz-Instituts für Deutsche Sprache (siehe auch Abbildung 6 in diesem Kapitel, http://www.ids-mannheim.de/kl/neoplots/ owid/179379.html, zuletzt eingesehen am 30.4.2021) und aus dem Korpus selbst ermittelten Ereignissen rund um das Thema „Burnout“, wie sie im Laufe dieses Kapitels und in Kap. 5.2 noch ausführlicher beschrieben werden. Des Weiteren wurden Texte beim „Wort \& Bild Verlag“, der die „Apotheken Umschau“ herausgibt, angefragt. Auf diese Anfrage erhielt die Verfasserin 10 Texte aus dem Zeitraum 2001-2012 zugeschickt, die ebenfalls in das Korpus eingeflossen sind. 
Themen behandeln, wie z. B. das „Helfer-Syndrom“239, Arbeitssucht, ${ }^{240}$ Stresserkrankungen bzw. der Zusammenhang von Arbeit, psychisch belastenden Arbeitsbedingungen, Stress und Krankheit ${ }^{241}$, das Konzept der „Gratifikationskrise“ “242 oder Konzepte, die teilweise als ähnlich etikettiert wurden wie das „Chronische Müdigkeits-Syndrom“243, aufgenommen und ein diachroner Strang aufgebaut werden. Diese auch bei der Zeitschrift „Psychologie Heute“ entstehende diachrone Übersicht bietet zudem Anhaltspunkte für die Rekonstruktion der gesellschaftlichen und politischen Faktoren, die auf den Burnout-Diskurs einwirken und die in Kapitel 5.2 ausführlich beschrieben werden. Im Folgenden sei an drei Beispielen ausschnittsweise verdeutlicht, inwiefern diese auch bei der weiteren Zusammenstellung des Korpus berücksichtigt wurden.

Erstens entwickelte sich durch das staatlich geförderte „Forschungsprogramm zur Humanisierung des Arbeitslebens“ Anfang der 1970er Jahre eine Diskussion über die Verbesserung von Arbeitsbedingungen und die aufgeworfene

Eine umfassendere digitale Eigen-Recherche im Archiv der Apotheken Umschau im Verlag war nicht möglich.

239 Vgl. z. B. den Beitrag „Unsere kranken Therapeuten“ von Schmidbauer (PH 9/1977: 5356, =Schmidbauer 1977; PH 2/2009: 62-67, =Schmidbauer 2009, und 6/2005: 64-69, =Speck/ Horsch 2005, im QV unter 8.1.7).

240 Vgl. PH 4/2000: 48-53 (= Robinson 2000, im QV unter 8.1.7). Auf der Symptomebene gibt es Überschneidungen und Unterschiede zu Artikeln, die `Burnout`-Symptome beschreiben. Arbeitssüchtige gingen auch ins Büro, „wenn es keinen ersichtlichen Grund dafür gibt“ (ebd.: 53).

241 In der AU sind die Themen „Stress“ und „mangelnde Entspannung“ wiederkehrende Themen, vgl. z. B. „Gibt es Stress wirklich“ in AU (1975: 21), „Erkennen Sie Ihren Streßfaktor?“ in AU (1995a: 64-65) oder „Stress? Ohne mich!“ in AU (2005a: 10-16); siehe auch AU (1982: 34); AU (1992: 23); AU (03/2012-A: 10-12, = Wolfrum/Jackus 2012); AU 12/2013-B: 10-16 (= Kandler-Schmitt 2013) und PH 2/2002: 26-27; PH 07/2000: 20-27 (= Ernst 2000); PH 2/2002: 26-27 (= Braun 2002). Auch die Verbindung von (arbeitsbedingtem) psychischem Stress zu somatischen Krankheiten, wie z.B. dem Herzinfarkt, kommt wiederholt vor, z. B. AU (1970) „Am Wochenende droht der Herzinfarkt“ (siehe auch AU (1998: 8-14), AU (2011a) oder PH 12/1980 „Ehrgeizig, überpünktlich, fleißig: Mit Volldampf in den Herzinfarkt“ (= Ernst 1980: 22); siehe auch PH 07/2000 (= Ernst 2000); alle Beiträge im QV unter 8.1.7.

242 Siehe z. B. den Beitrag „Die große Müdigkeit“ in PH 10/2002: 20-29, in dem das Konzept der „Gratifikationskrise“ auf S. 23 erläutert wird (= Krumpholz-Reichel 2002, im QV unter 8.1.7). 243 In PH 4/1992 wird von Hans Finck artikuliert, dass manche zunächst meinten, das Chronische Müdigkeits-Syndrom „,wäre eine Art psychophysiologisches Ausgebranntsein, ein ,Burnoutsyndrom', welches nur überdrehte ,Overachiever (Höchstleister) träfe“ (= Finck 1992: 25, im QV unter 8.1.7); es wurde teilweise ebenfalls als „Modekrankheit“ tituliert (vgl. AU 1995: 40-41); siehe zum CFS auch AU (1974: 10-11); AU (1999: 24-25); AU 10/2010: (= Reinbold 2010: 46-49); alle im QV unter 8.1.7. 
Frage, was gute Arbeit ausmache. ${ }^{244}$ Zweitens begann Anfang der 1970er Jahre der Arbeitsausschuss „AA5 Psychische Belastung“ des „Normenausschusses Ergonomie“ mit der Entwicklung einer „Norm zur psychischen Belastung und Beanspruchung“, auf deren Grundlage 1991 eine internationale Norm entstand, die im Jahr 2000 in das europäische und damit auch in das deutsche Normenwerk übernommen wurde (vgl. Nachreiner 2012: 9; siehe auch Kap. 5.2). Drittens hat das Thema „psychische Gesundheit“ Eingang in Gesetze und Vorgaben des Arbeitsschutzes gefunden, zunächst in der Bildschirmarbeitsverordnung 1996 und 2013 als Zusatz, in der vom Arbeitgeber durchzuführenden Gefährdungsbeurteilung zur „Verbesserung der Sicherheit und des Gesundheitsschutzes der Beschäftigten bei der Arbeit“ ( $§ 5$ Abs. 3 ArbSchG ${ }^{245}$ ).

Neben der „Apotheken Umschau“ und der „Psychologie Heute“ wurden des Weiteren Dossiers und Themenhefte weiterer populärwissenschaftlicher Magazine („Gehirn\&Geist“, „Focus Gesundheit“, „Geo Wissen“, „Spiegel Wissen“, „Stern“, alle im QV unter 8.1.7), die Internetportale „Wikipedia“, „Netdoktor“ und „Onmeda“ (im QV unter 8.1.8) sowie Medientexte überregionaler Zeitungen/ Magazine (Frankfurter Allgemeine Zeitung (FAZ), DER SPIEGEL, DIE ZEIT, und Süddeutsche Zeitung (SZ), im QV unter 8.1.6) in das fachexterne bzw. öffentliche Textkorpus mit aufgenommen. Die Printmedientexte wurden über die jeweiligen Archive der Zeitungen folgendermaßen recherchiert: Im Zeitraum 1980 bis 2000 wurden alle Texte der FAZ, des SPIEGELS und der ZEIT gelesen, welche die Suchwörter ${ }^{*}$ Burnout ${ }^{*}$ ODER ${ }^{*}$ Burn-out ${ }^{* 246}$ enthalten und die das Phänomen nicht nur erwähnen, sondern mindestens in einem Absatz bzw. einem Satz erläutern. Diese Menge wurde um ausgewählte Texte mit der Suchkombination „(ausgebrannt*

244 In der PH wird dieses Thema mehrfach aufgegriffen: (z. B. PH 2-1975: 9-14; 3-1975: 69-75; 9-1977: 40-46, diese Texte wurden ohne Autorennamen veröffentlich und finden sich im Quellenverzeichnis im Anhang unter PH (1975a), PH (1975b) und PH (1977), im QV unter 8.1.7).

245 Zum Wortlaut des Gesetzestextes siehe: https://www.gesetze-im-internet.de/arbschg/_5. html, zuletzt eingesehen am 17.11.2019; siehe als Beispiel für die Berichterstattung über „körperliche und psychische Belastungen bei den Arbeitnehmern an Bildschirmarbeitsplätzen“ den Artikel „Nö, der flimmert nicht“ von Marie-Yvonne Bogacki in einer Ressort-Beilage der Süddeutschen Zeitung vom 15. Juni 1994, S. 913 (= Bogacki 1994).

246 In den verschiedenen Archiven mussten unterschiedliche Trunkierungszeichen gesetzt werden. Im FAZ-Archiv lautete die Suchmatrix: $\left({ }^{*}\right.$ Burnout ODER Burnout $\left.{ }^{*}\right)$ ODER $\left({ }^{*}\right.$ Burn-out ODER Burn-out*), in der Süddeutschen Zeitung (burn-out* ${ }^{*}$ ODER burnout ${ }^{*}$ ), im SPIEGEL-Archiv wurde mit (burnout*, burn-out*) gesucht, DIE ZEIT, die im Deutschen Referenzkorpus archiviert ist, wurde über die Rechercheapplikation „Cosmas II“ des Leibniz-Instituts für Deutsche Sprache mit der Suchmatrix (*burn-out* oder burnout) erschlossen. Da man über Cosmas II nur Ausschnitte aus den gefundenen Texten einsehen kann, wurden die Gesamttexte daraufhin im Archiv der ZEIT nachrecherchiert (Stand 03/2017). 
ODER ausbr*) UND ( ${ }^{*}$ stress $^{*}$ ODER *arbeit* ${ }^{*}$ “247 sowie „sich/sO \&ausbrennen“248 ergänzt. Im ersten Jahrzehnt nach der Jahrtausendwende steigt die Anzahl der Texte, welche die Suchwörter Burnout/Burn-out enthalten, so stark, dass ein Weg gefunden werden musste, repräsentative Ausschnitte für eine qualitative Analyse in das Korpus miteinzubeziehen. Im Archiv der FAZ und des SPIEGELS wurden zunächst Texte zwischen 2001-2013/2015 249 für die Analyse markiert, die die Suchwörter ${ }^{\star}$ Burnout* $/{ }^{\star}$ Burn-out* im Titelbereich (und Vorspann) erwähnen (FAZ 99 Texte, SPIEGEL/SPIEGEL ONLINE ${ }^{250} 93$ Treffer). Im SPIEGEL und in DER ZEIT wurden vor 2000 ebenfalls Artikel zu den Suchwörtern *Burnout ${ }^{\star}$ / ${ }^{\star}$ Burn-out* (nicht nur im Titelbereich, sondern im gesamten Text) aufgenommen und in der SZ ergänzend Artikel, die „Burnout“ schwerpunktmäßig zum Thema haben. ${ }^{251}$ Das Archiv der Süddeutschen Zeitung „Librarynet“ wurde des Weiteren für eine Ressort-Recherche in den Zeiträumen 1.1.1992-31.12.2000 und 1.1.201131.12.2012 genutzt, ${ }^{252}$ da in diesem Archiv für den frühen Zeitraum auch die Landkreisausgaben mitberücksichtigt werden können, die interessante Meldungen zu Veranstaltungen vor Ort über „Burnout“ enthalten, und die Ressortverteilung in diesem Archiv gut auswertbar ist. Im Zeitraum 2009-2014 wurden schließlich noch ausgewählte Texte aus der Wochenzeitschrift DIE ZEIT extrahiert, die das Thema „Burnout“ im Artikel umfassend behandeln. ${ }^{253}$ Dabei wurden bei allen

247 Ohne das zusätzliche Suchwort Stress erhält man zu viele Treffer, die keinerlei Bezug zum untersuchten Gegenstand aufweisen, z. B. ausgebrannte Unfallfahrzeuge etc.

248 Vgl. die Recherche dazu im ZEIT-Korpus über das Deutsche Referenzkorpus (DEREKo). Siehe Fußnote 433 und 434 in Kapitel 6.1.2.

249 Aus dem Archiv von DER SPIEGEL/SPIEGEL ONLINE wurden die Treffer bis einschließlich 2013 bei der FAZ/faz.net bis 2015 gesichtet. Zusätzlich wurden einzelne Treffer aus 2016 und 2017 in das Analyse-Korpus aufgenommen (letzte Recherchen am 18.2.2017).

250 Im SPIEGEL wurde vor 2005 noch im Gesamttext gesucht und ab 2005 im Titelbereich.

251 Im SPIEGEL 13 Treffer, in der ZEIT 11 Treffer und in der SZ 11 Treffer.

252 Gesucht wurde mit folgender Suchsyntax: burn-out* ODER burnout* im Zeitraum 1.1.199231.12.2000 in den überregionalen und den Landkreisausgaben der Süddeutschen Zeitung und im Zeitraum 1.1.2011-31.12.2012 in der überregionalen Ausgabe. Die Landkreisausgaben als Quelle wurden bei dieser Recherche aufgrund der Fülle der Ergebnisse nicht mehr berücksichtigt. Die Ressort-Recherche und Auswertung im Archiv der SZ ist in Kapitel 6.2.4.1 beschrieben. Von dieser Recherche wurden nur einzelne Titel, die zitiert werden und das Thema „Burnout“ ausführlicher behandeln, in das Quellenverzeichnis als Einzeltitel übernommen (1992-2000: 11 Titel und 2011-2012: 9 Titel). Kurze Meldungen zu einem Vortrag oder Seminar, die nur für diese RessortRecherche genutzt wurden, sind also nicht einzeln im Quellenverzeichnis aufgeführt.

253 2009: 1 Treffer; 2010: 10 Treffer; 2011: 9 Treffer; 2012: 3 Treffer; 2013: 3 Treffer und 2014: 4 Treffer. Aufgrund der Fülle der Texte wurden hier nur noch Texte aufgenommen, die sich mit dem Thema „Burnout“ ausführlich auseinandersetzen. Texte, die das Wort Burnout nur am Rande verwenden und sonst über ein anderes Thema sprechen, wurden aussortiert. Damit sei nicht gesagt, dass diese ,beiläufige‘ Verwendung für die Analyse gänzlich uninteressant ist (diese Texte 
Zeitungen und Zeitschriften sowohl Print- als auch Onlinetexte aufgenommen. Pressetext-Zitate werden (außer bei Auszählungen wie z. B. für typische Berufsgruppen oder bei gesammelten Belegangaben zu sprachlichen Mitteln des Definierens) mit Autorenkennung im Fließtext oder in Fußnoten angegeben.

Die Zugriffspunkte ergaben sich aus einer Zeitverlaufsgrafik des OnlineWortschatz-Informationssystems Deutsch (OWID) zum Neologismus Burn-out des Leibniz-Instituts für Deutsche Sprache (IDS). Die Zeitverlaufsgrafik zeigt, „wie sich in den IDS-Textkorpora („Deutsches Referenzkorpus“ [DeReKo]) das Aufkommen einer neuen Zeichenkette sowie ihr Gebrauch über die Jahre hinweg spiegelt“. Für Burn-out zeigt sich ein erster deutlicher Anstieg 2006, ein starker Anstieg in den Jahren 2010, 2011, 2012 und ab 2013 kommt es zu einem Rückgang, 2015 wieder zu einem Zuwachs und erneuten Rückgang.

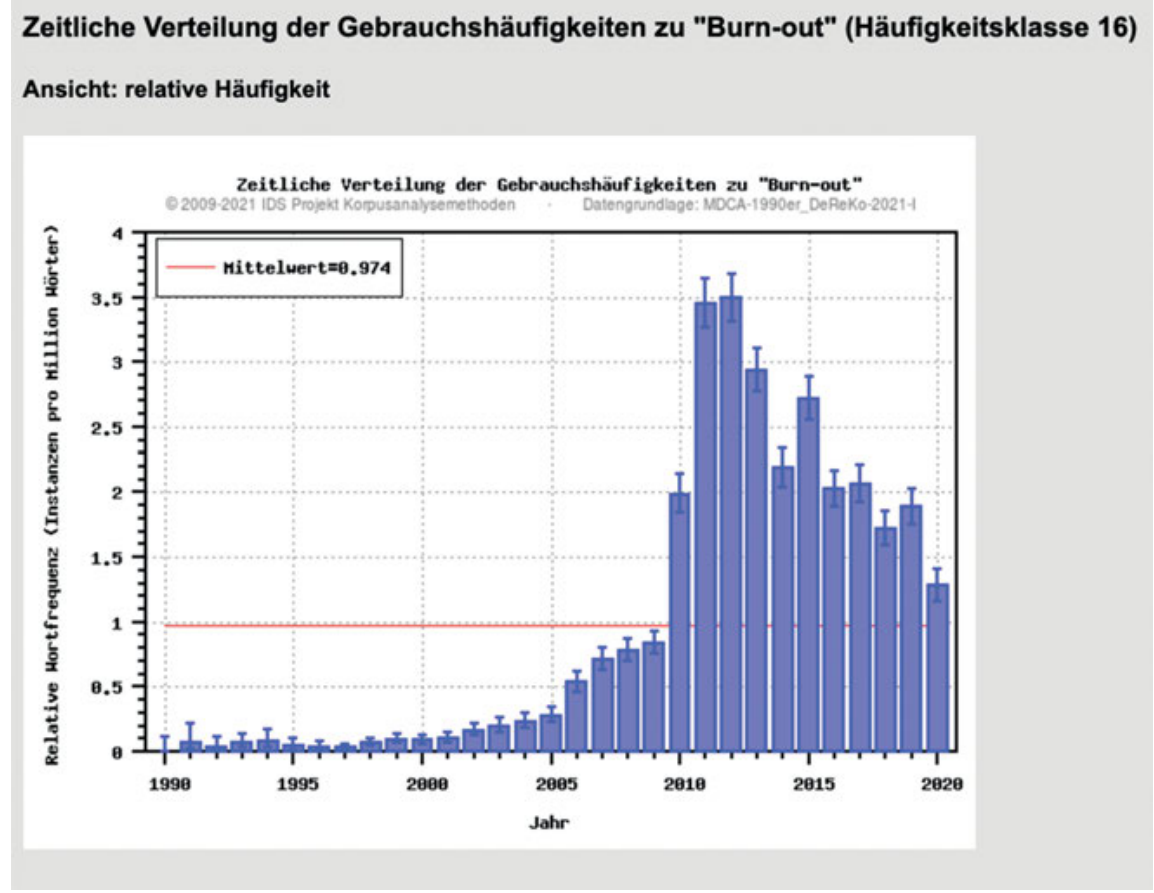

Abb. 6: Zeitverlaufsgrafik zum Neologismus Burnout des Leibniz-Instituts für Deutsche Sprache (http://www.ids-mannheim.de/kl/neoplots/owid/179379.html (zuletzt eingesehen am 30.4.2021)), (c) Leibniz-Institut für Deutsche Sprache 2009-2021.

sind daher auch Teil des SPIEGEL- und FAZ-Korpus und der in der Fußnote zuvor beschriebenen SZ-Ressort-Recherche). 
Des Weiteren kann man der Auswahl von Zugriffspunkten folgende durch Voranalysen ermittelte gesellschaftspolitische Entscheidungen und Ereignisse zum Thema „Burnout“ und angrenzender Themengebiete zugrunde legen:

- Erstens haben prominente Personen wiederholt eine mediale Berichterstattung über das Thema „Burnout“ ausgelöst: So zum Beispiel Sven Hannawald, der sich im April 2004 mit der Begründung, dass er „dem auf ihm lastenden Druck der letzten Zeit nicht mehr gewachsen“ ${ }^{\text {“254 }}$ sei, von den folgenden Trainingseinheiten abmeldete; 2006 wurde berichtet, Jürgen Klinsmann habe seinen Rücktritt damit erklärt, „dass er ,ausgebrannt“ sei““255; Ralf Rangnick trat am 22. September 2011 als Trainer des FC Schalke 04 wegen eines „Erschöpfungssyndroms“ zurück, was in der Berichterstattung zu verstärkter Reflexion über den Ausdruck und das Phänomen BURNOUT und das BURNOUT-SYNDROM geführt hat; ${ }^{256}$ oder Miriam Meckel, die in ihrem Buch „Brief an mein Leben“ (2010) ihre „Burn-out-Erfahrung“ ${ }^{257}$ beschrieb.

- Zweitens dienen Aktionen von gesellschaftspolitischen Akteuren als Orientierungspunkte, welche darauf zielen, das Thema „Burnout“ bzw. Fragen des Arbeitsschutzes auf die politische Agenda zu bringen, wie zum Beispiel durch Anfragen von (Bundestags-)Fraktionen an die Bundesregierung ${ }^{258}$ oder durch die Forderung der Berücksichtigung psychischer Belastung im Arbeitsschutzgesetz und die von Gewerkschaften wie der IG-Metall eingebrachte „Anti-Stress-Verordnung“(259.

254 Vgl. eine dpa-Meldung in der FAZ, in der berichtet wird: „Sven Hannawald befindet sich zur stationären Behandlung in einer Reha-Klinik“ (= dpa 2004a, FAZ vom 30.04.2004: 43).

255 Siehe dazu Kuhrt (2006), in: ZEIT ONLINE vom 20.07.2006.

256 Vgl. dpa (2011) in ZEIT ONLINE vom 22.09.2011, siehe im QV unter dpa (2011) im Abschnitt der Beiträge der ZEIT und ZEIT ONLINE. Über verschiedene Hochleistungssportler/innen mit „Burnout“ und anderen psychischen Erkrankungen wird auch in der Zeitschrift PH 9/2011 berichtet (= Ustorf 2011: 64-69).

257 Vgl. auch das Interview von Koelbl (2014) im ZEITmagazin Nr. 39/2014, 6. Oktober 2014, „Der Burn-out war ein Totalcrash“ (zuletzt eingesehen am 17.11.2019) und das Buch „Brief an mein Leben“ von Miriam Meckel (in der vierten Auflage aus dem Jahr 2013), siehe im QV unter 8.1.7 Meckel ( $\left.{ }^{4} 2013\right)$ unter der Rubrik „Weitere populärwissenschaftliche Zeitschriften und Bücher“.

258 Z. B. die Antwort der Bundesregierung auf die Kleine Anfrage der Abgeordneten Jutta Krellmann, Sabine Zimmermann, Dr. Martina Bunge, weiterer Abgeordneter und der Fraktion DIE LINKE zu „Psychische[n] Belastungen in der Arbeitswelt“ (siehe im QV unter 8.1.9 unter der Rubrik „Texte (gesundheits-)politischer Akteure“ Bundesregierung 2012a) oder die Antwort der Bundesregierung auf die Kleine Anfrage der Abgeordneten Beate Müller-Gemmeke, Markus Kurth, Brigitte Pothmer, weiterer Abgeordneter und der Fraktion Bündnis 90/Die GRÜNEN (2012) betreffend der „Aufsichtstätigkeit beim Arbeitsschutz“ insbesondere auch bezogen auf „[P]sychische Gefährdungen am Arbeitsplatz“ (siehe im Quellenverzeichnis Bundesregierung 2012b).

259 Darüber wird z. B. in der FAZ vom 09.01.2013 berichtet (= Schwenn 2013: 11, im QV unter 8.1.6), im manager magazin vom 28.09.2011 (= rei/dpa 2011, im QV unter 8.1.6), in der SZ vom 30.10.2012 
- Drittens wird die Berichterstattung zum Phänomen BuRnout von der Diskussion umrahmt, wo die Grenze zwischen psychischer Gesundheit und Krankheit verlaufe. Diese Frage wurde im Jahr 2013 im Zuge der Einführung der 5. Version des „Diagnostical and statistical manual of mental diseases“ (DSM-5) diskutiert. ${ }^{260}$

Auf der Basis der Zeitverlaufsgrafik aus OWID und diesen Ereignissen wurde folglich entschieden, welche Jahre im Fokus der Medienanalyse stehen. Das bedeutet nicht, dass in anderen Jahren unbedeutende Beiträge zum Thema „Burnout“ in den Medien erschienen sind, und es wird kein Anspruch auf Vollständigkeit der Zusammenstellung der politischen und gesellschaftlichen Ereignisse zum Thema „Burnout“ erhoben. ${ }^{261}$ Für die Auswahl repräsentativer Ausschnitte spricht jedoch, dass auf diese Weise Verbindungslinien im Korpus hergestellt werden können, welche eine Vergleichbarkeit zwischen den Teilkorpora ermöglichen.

\subsection{Fachkulturelle, -politische, sozialpolitische und gesellschaftliche Rahmenbedingungen des Burnout-Diskurses}

Bevor die zu untersuchenden Texte in Kapitel 5.3 aus einem varietätenlinguistischen Blickwinkel heraus fokussiert werden, gibt dieses Kapitel einen Überblick über die fachkulturellen, fachpolitischen und sozialpolitisch-gesellschaftlichen Rahmenbedingungen, vor denen die Texte über das Phänomen Burnout entstehen. Einige dieser Entwicklungen wurden in Kapitel 5.1 im Zuge der Erläuterung zur Zusammenstellung der Korpora schon erwähnt. Es wird im Folgenden darum gehen, das politische und wirtschaftliche Klima in der Entstehungszeit des Burnout-Diskurses seit Mitte der 1970er Jahre in groben Zügen zu skizzieren und den Gang politisch geförderter Forschungsprogramme und der Forschungsfelder, aus denen die Burnoutforschung zum Teil hervorgegangen ist, zu umreißen.

(Bohsem/Haas 2012) und im Dt. Ärztebl. (siehe Bühring 2014: A-1630, im QV unter 8.1.4).

260 Darüber berichtet zum Beispiel DER SPIEGEL am 12.07.2012 unter dem Titel „Umstrittenes Psychologiewerk: Katalog der Störungen“ (= Hauschild 2012, im QV unter 8.1.6).

261 So könnte man beispielsweise auch die Liste der Prominenten, die über ihre `Erschöpfung, bzw. ihr `Burnout` sprechen, erweitern: 2003 gibt es Berichte darüber, dass der Fußballer Sebastian Deisler wegen eines „Burnout-Syndroms“ in Behandlung sei (siehe im QV SPIEGEL ONLINE 2003a und 2003b), im selben Jahr wird „Burnout“ auch in Bezug auf Harald Schmidts Pause von der Late-Night-Show attestiert (SPIEGEL ONLINE 2003c und 2003d) und Tim Mälzer spricht 2009 über seinen Burnout drei Jahre zuvor (siehe im QV unter 8.1.6 SPIEGEL ONLINE (2009), 22.03.2009). 
Will man beschreiben, vor welchem Hintergrund sich der Diskurs zum Thema „Burnout“ entwickelt, so erweist sich einerseits ein Blick auf gesellschaftlichpolitische Ereignisse - insbesondere im Bereich wirtschaftlicher Entwicklungen und ihrer Wechselwirkungen mit Arbeitsmarkt, Arbeitspolitik und Wissenschaft als aufschlussreich, und andererseits geben sachverwandte Forschungsbereiche und die fachliche und fachpolitische Entwicklung der mit diesen Themen befassten medizinisch-psychologischen und -soziologischen Spezialfächer Hinweise auf den historisch-fachkulturellen Hintergrund. ${ }^{262}$

Die sozial-, gesellschafts-, politik- und fachgeschichtlichen Entwicklungen der Jahrzehnte 1970-2015 können hier nicht grundständig erörtert werden. Dazu müssten alle gesellschaftlich-kulturellen und fachlichen Dimensionen einzeln in ihrer historischen Entwicklung betrachtet werden. Die folgenden Erläuterungen sollen jedoch dazu dienen, einen Rahmen zu skizzieren, vor dem sich das Thema „Burnout“ ab Mitte der 1970er Jahre entfalten konnte.

Werner Bührer (2002) beschreibt in einem Informationsheft der Bundeszentrale für politische Bildung die wirtschaftliche Entwicklung der 1970er Jahre in der Bundesrepublik folgendermaßen:

Mit den 1970er Jahren endet das deutsche Wirtschaftswunder. Die Binnennachfrage ist gesättigt, neue Produktionsverfahren verringern den Arbeitsaufwand, die Arbeitslosenzahlen steigen und die Einkommen sinken. Durch u. a. Senkung der Staatsverschuldung, steuerliche Entlastung der Unternehmen und Reduzierung der staatlichen Ausgaben für sozialpoitische [sic] Maßnahmen versucht die sozialliberale Koalition der Wirtschaftskrise zu begegnen. ${ }^{263}$

Wie im Zitat angedeutet, musste die deutsche Politik seit Anfang der 1970er Jahren auf die erste wirtschaftliche Krise nach dem sog. Wirtschaftswunder reagieren. Die Inflationsrate stieg 1973 bis zur Marke von sieben Prozent und die „Überwindung der Arbeitslosigkeit“ wurde zu einem Dauerproblem seit Mitte der 1970er Jahre (vgl. ebd.). Von dieser Arbeitsmarktkrise war auch der Sektor der psychosozialen Berufe betroffen, der zuvor einen Aufschwung erlebt hatte. ${ }^{264}$

262 Zur Entwicklung und den Besonderheiten medizinischer und psychologischer Wissensbildung im Allgemeinen siehe Kapitel 3.2 dieser Arbeit.

263 Der Text von Bührer ist abrufbar unter: http://www.bpb.de/izpb/9748/wirtschaftlicheentwicklung-in-der-bundesrepublik (zuletzt eingesehen am 17.11.2019).

264 Zunächst war der Arbeitssektor der sog. helfenden Berufe gestiegen. In diese Zeit fallen des Weiteren die Begriffe `Pflegenotstand`, >Reform der Psychiatrie` und `Zunahme der Intensivmedizin`. Ab Mitte der 1970er Jahre wird allerdings auch für diesen Sektor ein Überangebot an Bewerberinnen und Bewerbern im Vergleich zu offenen Stellen gemeldet (vgl. dazu einen Artikel im SPIEGEL vom 10.03.1975 (siehe im Quellenverzeichnis unter DER SPIEGEL 1975). 
Dieter Kleiber und Dirk Enzmann (1986) beziehen diese Lage sogar in ihre „Überlegungen zum BURNOUT in helfenden Berufen“ mit ein:

Die Entwicklungen des Arbeitsmarktes für die psychosozialen Berufe haben nun ganz handfeste Auswirkungen, die ein Ausbrennen der (Noch-)Arbeitsplatzinhaber fördern können. Naheliegend ist, daß die Konkurrenz untereinander wächst und damit die Konflikt- und Kooperationsfähigkeit innerhalb der psychosozialen Einrichtungen geschwächt wird. [...] Schließlich geht mit der Arbeitslosigkeit ein - vielleicht der - entscheidende Bewältigungsmechanismus von Burnout verloren; der Arbeitsplatzwechsel.

(Kleiber/Enzmann 1986: 57, siehe im QV unter 8.1.5)

Inwiefern das Aufkommen des Burnoutdiskurses durch diese arbeitsmarktpolitischen und wirtschaftlichen Faktoren beeinflusst wurde, ist nicht mit Sicherheit auszumachen. Man kann nur von einer Gleichzeitigkeit dieser Ereignisse sprechen. Das Zitat von Kleiber/Enzmann zeigt allerdings, dass diese Parallelen gezogen wurden und dass Angehörige von psychosozialen Berufen begonnen hatten, verstärkt über die Charakteristika, Probleme und die Zukunft ihres Berufsstands zu reflektieren. ${ }^{265}$

Wie schon in Kapitel 5.1.3 erwähnt, gewann auch die Forschung rund um das Thema „psychische Belastung und Beanspruchung bei der Arbeit“ in Deutschland seit den 1970er Jahren durch das 1974 begonnene, von den damaligen Bundesministerien für „Arbeit und Sozialordnung“ und „Forschung und Technologie“ geförderte Programm „Forschung zur Humanisierung des Arbeitslebens“ in der wissenschaftlichen und gesellschaftlich-politischen Reflexion an Bedeutung. ${ }^{266}$ Das Programm hatte sich zum Ziel gesetzt, Möglichkeiten zu untersuchen, wie die „Arbeitsbedingungen stärker als bisher den Bedürfnissen der arbeitenden Menschen angepaßt“267 werden könnten. Dabei wurde unter anderem die Frage diskutiert, in welcher Weise die „Teilnahme an stupiden automatisierten Ferti-

265 In diese Zeit fallen auch die ersten deutschen Übersetzungen von englischsprachigen Publikationen über das Thema „Burnout“, die teilweise dezidiert im sog. Helferbereich angesiedelt waren, z. B. Freudenberger/Richelson (1980a und b); Pines/Aronson/Kafry (1983); Edelwich/Brodsky (1984) (siehe im QV unter 8.1.5); Ein Interview in PH 11/1983 mit Christina Maslach (= Ernst 1983) und eine Übersetzung von Aronson/Pines/Kafry in PH 10/1983, siehe im QV unter 8.1.7.

266 Das staatlich geförderte Forschungsprogramm wurde 1989 zum Programm „Arbeit und Technik“ umgestaltet. Im Magazin PH erscheinen in den 1970er Jahren mehrere Artikel, die dem Thema „Humanisierung der Arbeitswelt“ gewidmet sind (z. B. PH 1975a: 9-14; PH 1975b: 69-75; PH 1977: 40-46, siehe im QV unter 8.1.7). Vgl. den Fachartikel von Hacker (1991 in ZfAO, im QV unter 8.1.4), der sich kritisch mit dem „Belastungs-/Beanspruchungskonzept“ auseinandersetzt. 267 So zitiert in einem Artikel aus DER SPIEGEL, Nr. 19/1974: 57f. (= Der SPIEGEL (1974) im QV unter 8.1.6). Vgl. auch Salfer/Furmaniak 1981: 237 im Literaturverzeichnis 8.2. 
gungsprozessen“ 268 den Grad psychischer Belastung beeinflusst. Eine verwandte Fragestellung - wie Unterforderung sich auf die Arbeitszufriedenheit und psychische Gesundheit auswirkt - wird in den letzten Jahren unter dem Stichwort Bore-out diskutiert. ${ }^{269}$

Zeitlich parallel zu diesem Humanisierungsprogramm setzte Anfang der 1970er Jahre die Entwicklung einer Norm zur psychischen Belastung und Beanspruchung ein, deren erste Fassung DIN 33405 allerdings erst 1987 veröffentlicht werden konnte, da die im Normenausschuss vertretenen Tarifvertragsparteien, u. a. Vertreter von Arbeitnehmer- und Arbeitgeberseite, „die Relevanz oder auch Brisanz der Norm für den Arbeitsschutz relativ hoch einschätzten“ (Nachreiner 2012: 9). 1991 wurde eine an die deutsche Norm anknüpfende internationale Norm ISO 10075: 1991 publiziert und 2000 wurde sie in das europäische und damit auch wieder in das deutsche Normenwerk als DIN EN ISO 10075-1:2000:11 unter dem Namen „Ergonomische Grundlagen bezüglich psychischer Arbeitsbelastung Teil 1: Allgemeines und Begriffe“ übernommen (ebd.: 10). ${ }^{270}$ Parallel zu dieser terminologisch ausgerichteten ersten Norm wurde 1996, so fasst Nachreiner weiter zusammen, eine zweite Norm zur Arbeitsgestaltung, d.i. ISO 10075-2 „Ergonomic principals related to mental workload - Part 2: Design principles“ entwickelt, die 2000 trotz des Widerstands von Arbeitgeberseite in das deutsche Normenwerk übernommen wurde, da die Norm auf europäischer Ebene überwiegend Zustimmung erhalten hatte (ebd.: 11). Im Jahr 2004 wurde schließlich eine dritte Norm zu den „Anforderungen an Messverfahren“ veröffentlicht „DIN EN ISO 10075-3“ (ebd.: 12). Diese Normentwicklung ist für die vorliegende Arbeit auch vor dem Hintergrund interessant, dass eine personelle Verbindung zum Burnoutdiskurs besteht. Friedhelm Nachreiner, der in den Ausschüssen des Normenausschusses Ergonomie auf nationaler und internationaler mitgearbeitet hat, ${ }^{271}$ entwickelte zusammen mit Evangelia Demerouti den Oldenburg Burnout Inventory (OLBI)

268 Vgl. die Anmerkung zuvor.

269 Vgl. dazu den Artikel auf ZEIT ONLINE „Krank vor Langeweile“ vom 26.06.2010, (= Dpa (2010) im Quellen-Abschnitt von DIE ZEIT und ZEIT ONLINE unter 8.1.6) und „,Langeweile ist kein schickes Leiden'. Psychotherapeut Wolfgang Merkle über das Bore-Out-Syndrom, den kleinen Bruder des Burn-Out“ vom 25.04.2010 in der FAZ (= Fritzen 2010: 14); siehe auch jol/gms (2007, SPIEGEL ONLINE am 05.11.2007) „Diagnose ,Boreout‘: Bürostress durch Langeweile.“

270 DIN 33405 wurde damit zurückgezogen (Nachreiner 2012: 10). Zur Entwicklung der Norm im Bereich der psychischen Belastung siehe im Überblick auch Nachreiner (2012: 8ff.) im Literaturverzeichnis 8.2.

271 Vgl. die Publikation „Psychische Belastung und Beanspruchung am Arbeitsplatz. Inklusive DIN EN ISO 10075-1 bis -3.“, die u. a. von Evangelia Demerouti und Friedhelm Nachreiner herausgegeben wurde. Die Mitarbeit von F. Nachreiner bei Normungsaktivitäten ist auch unter folgenden Link auf der Seite der „Gesellschaft für Arbeits-, Wirtschafts- und Organisationspsychologische For- 
(Demerouti/Nachreiner 1998) $)^{272}$, eines der ersten Messinstrumente aus dem deutschsprachigen Raum in ,Konkurrenz' zum Maslach Burnout Inventory (MBI).

Im Jahr 1998 wurde in der Bundesrepublik die neunte Fassung des internationalen Diagnoseschlüssels der WHO (ICD-9) durch die revidierte zehnte Fassung (ICD-10) abgelöst. ${ }^{273}$ In der ICD-10 erscheint „Burn-out“ das erste Mal als „Zustand der totalen Erschöpfung“ unter dem Code Z73.0 als Zusatzdiagnose. ${ }^{274}$ Dies könnte als Anzeichen seiner Institutionalisierung interpretiert werden. Andererseits erscheint Burnout weder in der vierten noch in der im Jahr 2013 herausgegebenen fünften Fassung des „Diagnostic and Statistical Manual of Mental Disorders" (DSM), ein Diagnoseleitfaden, der auch hierzulande eine wichtige Rolle für die Definition und Diagnostik von psychischen Störungen spielt. ${ }^{275}$ Dass sich das Konzept >BURNouT` in der Fortbildungs- und ärztlichen Praxis in Deutschland $\mathrm{zu}$ einem gewissen Grad allerdings doch etabliert hat, zeigt wiederum ein Übersichtsartikel von 2014 in der Zeitschrift „Deutsche medizinische Wochenschrift“: Dort können Ärzte und Ärztinnen durch das Beantworten von Fragen zum Beitrag „Das Burnout-Syndrom: Prävalenz, Symptome, Differenzialdiagnose und Therapie“ Fortbildungspunkte sammeln (vgl. Kissling/Mendel/Förstl 2014 ${ }^{276}$ ).

Betrachtet man die Entwicklungen bestimmter Fächer der angewandten medizin-psychologischen Forschung seit den 1970er Jahren, so fällt auf, dass die Stressforschung ${ }^{277}$, welche dem Phänomen BuRnout thematisch sehr nahe steht, ${ }^{278}$ durch das bis heute einflussreiche transaktionale Stressmodell von Richard S. Lazarus und Mitarbeiterinnen und Mitarbeitern neu belebt wurde (vgl. Lazarus/Launier 1981; Lazarus/Folkman ${ }^{9} 1996$; Stier-Jarmer/Oberhauser

schung e.V.“ einsehbar: http://www.gawo-ev.de/cms2/index.php?page=prof-dr-nachreiner\&phpMyAdmin=8b6ed5803bbabc8d5f96599c9c6997ad (zuletzt eingesehen am 17.11.2019).

272 Im QV unter 8.1.5, Zeitschrift für Arbeitswissenschaft.

273 Vgl. dazu die Angaben auf dem Portal des Deutschen Instituts für medizinische Dokumentation und Information (DIMDI) unter: https://www.dimdi.de/dynamic/de/klassifikationen/icd/ icd-10-who/historie/versionsverlauf/, zuletzt eingesehen am 17.11.2019. Vgl. zur Entstehung der ICD-Versionen auch die Informationen der WHO unter: http://www.who.int/classifications/icd/ en/ (zuletzt eingesehen am 17.11.2019).

274 Diese ältere Version der ICD-10 ist einsehbar unter: https://www.dimdi.de/static/de/klassifikationen/icd/icd-10-who/kode-suche/htmlamtl/ (zuletzt eingesehen am 17.11.2019).

275 Siehe im QV unter Saß u. a. 2003 und Falkai/Wittchen/Döpfner u. a. (2015) im Abschnitt 8.1.3. 276 Siehe im QV unter Abschnitt 8.1.4 bei „Deutsche medizinische Wochenschrift“.

277 Die Stressforschung selbst geht einerseits auf Arbeiten des Mediziners Walter B. Cannon (1914) und andererseits entscheidend auf Hans Selye zurück (siehe Cannon (1914) und Selye (1981, im QV unter 8.1.5 und Selye ${ }^{2}$ 1988) und vgl. Semmer (1992: 744, im QV unter 8.1.2).

278 Dies zeigt sich u. a. in dem Befund, dass occupational stress in der Datenbank PSYNDEX als eines der häufigsten sachverwandten Schlagwörter angegeben wird (vgl. Kap. 5.1.2 dieser Arbeit und im QV 8.1.3). 
et al. 2016) ${ }^{279}$. Die Verbindung von körperlichen und psychosozialen Faktoren wurde zudem in der erstarkenden Fachrichtung der Psychosomatik erforscht ${ }^{280}$, und mit der Approbationsordnung von 1970 wurden die Fächer „Medizinische Psychologie“ und „Medizinische Soziologie“ als Pflichtfächer im Medizinstudium verankert. ${ }^{281}$

Aus dieser Zeit stammen beispielsweise Studien zum Zusammenhang zwischen Herz-Kreislauf-Erkrankungen, Stress und dem sog. „Typ-A-Verhalten“, „ein Verhaltensmuster, das durch ehrgeiziges Leistungsstreben, Konkurrenzorientierung, Ungeduld, Zeitdruck, Feindseligkeit, Ärger, Aggressivität und explosive Sprechweise gekennzeichnet“ sei (Schwarzer ${ }^{3} 2004: 1$ ). In der aktuellen (populärwissenschaftlichen) Berichterstattung werden diese Annahmen teilweise relativiert (vgl. ebd.; Heitkämper 2017). ${ }^{282}$ Studien dieser Art fanden damals jedoch auch Verbreitung in der nichtakademischen Öffentlichkeit. ${ }^{283}$ Dies ist deshalb interessant, da im Burnout-Diskurs einige dieser Verhaltens- und Persönlichkeitsattribute (z. B. Ehrgeiz und Leistungsstreben) aufgegriffen und mit der Entwicklung von BuRnout-Symptomen in Verbindung gebracht werden. Parallel dazu differenzierten sich einzelne angewandte Teildisziplinen weiter aus, wie zum Beispiel die Psychosomatik, Arbeits- und Organisationspsychologie ${ }^{284}$, die medizinische Soziologie, die medizinische Psychologie sowie die Gesundheitspsychologie. Im

279 Siehe Lazarus/Folkman ( $\left.{ }^{9} 1996\right)$ und Lazarus/Launier (1981) im Literatur-Verzeichnis unter 8.2 und Stier-Jamer u. a. (2016) im QV unter 8.1.4 unter „Dt. Ärzteblatt“.

280 Der Aufschwung dieser Fachrichtung zeigte sich u. a. daran, dass im April 1950 „die erste klinische Universitätseinrichtung für psychosomatische Medizin in Deutschland“ in Heidelberg unter der Leitung von Alexander Mitscherlich eröffnet wurde (vgl. Freimüller 2019: 201). Zur Entwicklung der Psychosomatik im 20. Jahrhundert siehe die monografisch angelegte Aufsatzsammlung „Auf der Suche nach einer anderen Medizin. Psychosomatik im 20. Jahrhundert“, hrsg. von Alexa Geisthövel und Bettina Hitzer (Geisthövel/Hitzer 2019a) sowie die Einleitung zu diesem Band (Geisthövel/Hitzer 2019b).

281 Siehe in der Approbationsordnung von 1970, 3. Abschnitt § 22, einsehbar unter: https://www. bgbl.de/xaver/bgbl/start.xav?start=\%2F\%2F*\%5B\%40attr_id\%3D\%27bgbl170098.pdf\% 27\%5D\#__bgbl_\%2F\%2F*\%5B\%40attr_id\%3D\%27bgbl170098.pdf\%27\%5D_1491226457152, (Stand 26.11.2019, im QV unter 8.1.9).

282 Vgl. den Beitrag „Die Herz-Seele-Connection“ von Edith Heitkämper in PH 3/2017: 60 (siehe im QV 8.1.7). Die Übersichtsarbeit von Ladwig/Lukaschek/Baumert (2015) im Dt. Ärztebl. (im QV unter 8.1.4) stellt jüngere Studien vor, die Faktoren wie z. B. „psychomentale Stressbelastung“, „Mobbing“ oder „Überforderung durch Mehrarbeit/Überstunden“ als „Risiko für das spätere Auftreten von einer Koronaren Herzkrankheit (KHK)“ untersuchen (ebd.: 30).

283 Vgl. z. B. Berichte in der AU „Sind Sie Typ A oder eher Typ B?“ (1970b: 1-2); „Am Wochenende droht der Herzinfarkt“ (AU 1970a, siehe im QV unter 8.1.7).

284 Die Anfänge der Arbeits- und Organisationspsychologie als angewandter psychologischer Teildisziplin reichen bis zum Beginn des 20. Jahrhunderts zurück. Siehe im Überblick dazu Kanning (2007: 27ff., im QV 8.1.2). 
Bereich psychosozialer Berufspraxis kommt es zudem zur Entstehung von Balintund Supervisionsgruppen. ${ }^{285}$ Die Entwicklung dieser >Denk- und Praxisstile bezogen auf den Themenbereich von (psychosozialer) Arbeit, Leistung und (psychischer) Gesundheit sei an der Entwicklung des Fachs der Arbeits- und Organisationspsychologie illustriert: Schwerpunkte der „industriellen Psychotechnik“286 bzw. Arbeitspsychologie waren in der ersten Hälfte des 20. Jahrhunderts u. a. die Eignungsdiagnostik (auch für militärische Zwecke) und Zeit- und Bewegungsstudien zur Optimierung der Produktionsprozesse „an der Schnittstelle zwischen Mensch und Maschine“ (Kanning 2007: 30) im Sinne einer „wissenschaftlichen Betriebsführung nach Taylor und der industriellen Psychotechnik“ (Nerdinger ${ }^{3} 2014$ : 22). Der Taylorismus kam jedoch in die Kritik. So stand auch bei der Einsetzung des oben erwähnten staatlichen Forschungsprogramms zur „Humanisierung des Arbeitslebens“ die Auseinandersetzung mit der tayloristisch-fordistischen Organisation von Arbeit im Mittelpunkt. Daran wurde bemängelt, dass durch die einseitigen Optimierungsbestrebungen zwischen Mensch und Maschine (z. B. am Fließband) die Arbeit immer monotoner und soziale Interaktionen zwischen den Mitarbeiterinnen und Mitarbeitern und Vorgesetzten $\mathrm{zu}$ wenig beachtet würden. Arbeiten, die „das soziale Verhalten in Organisationen“ (Nerdinger ${ }^{3} 2014:$ 22), „Führungsforschung, Arbeitszufriedenheit, Konflikte am Arbeitsplatz“ (Kanning 2007: 31) stärker in den Blick nahmen, kamen schließlich in den 1930er Jahren in den USA im Zuge der sog. Human-Relations-Bewegung auf und erhielten mit der sich in den 1960er Jahren etablierenden Organisationspsychologie eine eigene mit der Arbeitspsychologie eng verbundene Teildisziplin (vgl. Nerdinger ${ }^{3} 2014:$ 22; Kanning 2007: 31.f). ${ }^{287}$ Das erste deutschsprachige Lehrbuch zur Organisations-

285 Ein Beitrag aus dem SPIEGEL mit dem Titel „Hölzerne Seelen“ (DER SPIEGEL 39/1976: 234-238) behandelt die Praxis der Balint-Gruppen (siehe im QV unter 8.1.6). Aus dem Jahr 1995 gibt es einen Bericht zu einer Studie am Universitätsklinikum Heidelberg zur Evaluation von einem Jahr Stationsteam-Supervision (siehe Werner 1998 im Dt. Ärztebl., im QV unter 8.1.4).

286 Der Begriff „Psychotechnik“ geht auf den Psychologen William Stern (1871-1938) zurück, der damit Anspruch auf eine anwendungsorientierte Psychologie erhob, die „mit dem wahren Leben zu tun haben und überdies praktisch nützlich sein“ sollte (Kanning 2007: 29). Die „industrielle Psychotechnik“, eine Bezeichnungsspezifizierung von Walther Moede, bezog sich auf die „Anwendung der Psychologie in Produktionsbetrieben“, die sich insbesondere auch auf Arbeiten des Psychologen Hugo Münsterberg stützte (Nerdinger ${ }^{3} 2014:$ 21). Die Bezeichnung Psychotechnik wurde in den 1940er Jahren von der Bezeichnung Arbeitspsychologie mehr und mehr abgelöst (vgl. Kanning 2007: 31); (siehe im QV 8.1.2).

287 Die einseitige Darstellung vom inhumanen „Taylorismus“ gegenüber der darauf kritisch reagierenden Human-Relations-Bewegung wird nach neueren Studien jedoch ebenfalls relativiert (Nerdinger ${ }^{3}$ 2014: 22, siehe im QV 8.1.2). Letztere habe auch zu negativen Konsequenzen geführt z. B. in Bezug auf die Festigung autoritärer Strukturen in Unternehmen, und Hugo Münzberg, als 
psychologie von Lutz von Rosenstiel, Walter Molt und Bruno Rüttinger erschien nach Nerdinger ( ${ }^{3} 2014:$ 22) im Jahr 1972. 1985 wurde in der Deutschen Gesellschaft für Psychologie schließlich die Fachgruppe „Arbeits- und Organisationspsychologie“ gegründet (Kanning 2007: 32).

Dieser kurze Abriss zur Geschichte der Arbeits- und Organisationspsychologie zeigt eine frühe Internationalisierung und Rezeption insbesondere angloamerikanischer Fachliteratur im deutschsprachigen Forschungsraum, wobei deutsche Veröffentlichungen, vor allem aus der Konsolidierungsphase des Fachs Psychologie (Ende des 19. und Anfang des 20. Jahrhunderts) von amerikanischer Seite aus rezipiert und zitiert wurden. ${ }^{288}$

In den 1970er Jahren setzten im Fach Psychologie eine Debatte über die internationale Rolle der deutschsprachigen psychologischen Forschung und der sog. „Sprachenstreit“ ein (vgl. Schui 2004: 4). Dabei geht es bis heute um die Frage, in welchem Umfang Wissenschaftlerinnen und Wissenschaftler auf Deutsch und/ oder Englisch publizieren, wobei es große Unterschiede zwischen den psychologischen Teilfächern bezüglich des englischsprachigen Publikationsanteils gibt. Für die angewandten Fächer (Arbeits- und Organisationspsychologie, Pädagogische Psychologie, Klinische Psychologie und Psychotherapeutische Schulen) kommt Schui im Jahr 2004 zu dem Schluss, dass „Literatur und Lehre primär deutschsprachig“ seien. Im 2016 vom Leibniz-Zentrum für Psychologische Information und Dokumentation (ZPID) veröffentlichten Monitor zum Publikationsjahr 2014 weisen wiederum die „Anwendungsfächer die niedrigsten englischsprachigen Anteile“ auf: die Arbeits- und Organisationspsychologie mit einem englischsprachigen Anteil von 19 \%, die Pädagogische Psychologie mit 22,9 \% und die Klinische Psychologie mit 33,2 \% ${ }^{289}$. Im Gegensatz dazu kommen die Fächer der Allgemeinen Psychologie und Biopsychologie auf einen englischsprachigen Pub-

einer der Wegbereiter der industriellen Psychotechnik, habe bereits „die Bedeutung der sozialen Beziehungen im beruflichen Alltag für die Arbeitsleistung“ erkannt (ebd.).

288 Vgl. die bibliografische Analyse zur „Rezeptionsgeschichte von Fachliteratur aus der deutschsprachigen Psychologie in der amerikanischen Psychologie (et vice versa)“ am Beispiel von Kongress-Eröffnungsvorträgen von APA- und DGPs-Präsidenten im 20. Jahrhundert (Krampen/Montada/Schui u. a. 2002).

289 Diese $33 \%$ müssen noch einmal aufgespalten werden, da die Klinische Psychologie in der verwendeten Nomenklatur der Studie keine eigenständige Kategorie darstellt. Die Autoren haben sie ,aus den Klassifikationen Psychische und Physische Störungen sowie Behandlung und Prävention“ mit logischem ODER zusammengesetzt. Der stärker grundlagenorientierte Forschungsbereich „Psychische und physische Störungen“ hat mit 43,7 \% einen deutlich höheren Anteil englischsprachiger Publikationen als der Bereich „Behandlung und Prävention“ mit 19,2 \%. (Schui/Krampen 2016: 4). Online abrufbar unter: http://dx.doi.org/10.23668/psych archives.4914, zuletzt eingesehen 13.7.2021. 
likationsanteil von fast $90 \%$ und die Sozialpsychologie von $72 \%$ (Schui/Krampen 2016: 4f.). Aufgrund dieser Verteilung wurde das fachliche Korpus vorwiegend aus deutschen Publikationen zusammengestellt, wobei englischsprachige Literatur, wie in Kapitel 5.1.2 erläutert, einbezogen wurde, wenn sie im fachlichen, aber auch öffentlichen Diskurs wiederholt bzw. an exponierter Stelle zitiert wird.

\subsection{Erscheinungsformen des fachlichen und öffentlichen Diskursstrangs}

\subsubsection{Vorbemerkung}

Für Foucault stehen hinter diskursiven Formationen soziale und diskursive Regeln und Regelmäßigkeiten des Erscheinens von Gegenständen bzw. „Aussagen“ oder Zeichenfolgen an der Oberfläche des Diskurses. ${ }^{290}$ Dieser Begriff des diskursiven >Erscheinens` von Zeichenfolgen wird in den folgenden Kapiteln mit dem varietätenlinguistischen Beschreibungskonzept der „Erscheinungsformen der deutschen Sprache“ verbunden (Steger 1988; Felder 2016). Ein besonderes Augenmerk liegt hierbei auf fach- und vermittlungskommunikativen sowie textlinguistischen bzw. -sortenspezifischen Aspekten (vgl. Franke 1997, Liebert 2002).

Die folgenden Unterkapitel haben das Ziel, die Texte der verschiedenen Teilkorpora aus einer varietäten- und soziolinguistischen Perspektive heraus zu charakterisieren. Die im vorangehenden Kapitel beschriebenen fachkulturellen sowie fach- und sozialpolitischen Hintergründe des Burnout-Diskurses (Kap. 5.2) sollen „durch innersprachliche und außersprachliche Bestimmungsmerkmale sprachlicher Variationen“ (Felder 2016: 1) und der varietätenlinguistischen Einordnung verbunden werden. Dafür werden orientiert am „Varietäten-Auge“ von Felder (2016: 128ff.) inner- und außersprachliche Merkmale zusammengeführt mit Perspektive auf: die „kommunikative Reichweite der Ausdrücke“, die „funktionale Reichweite der Semantik/Inhalte“, die „Medialität der Zeichen bzw. Zeichentypen“, die „historischen Zeitstufen“ (Felder 2016: 59), „Raum, Zeit und Ort“, „soziale Gruppierung“ und „Situation“ (Felder 2016: 114ff.; 128).

Die wichtigste Dimension zur Unterscheidung der Texte der verschiedenen Teilkorpora, die in den verschiedenen Teilkorpora in der vorliegenden Arbeit zusammengefasst werden, ist die „funktionale Reichweite der Inhalte“ (Felder

290 Siehe dazu ausführlich Kap. 4.1.3 zu >Praxis` und (nicht-)diskursive Praktiken « bei Foucault; vgl. Foucault [1969] 1981: 61ff.; Foucault [1970] 1993: 17ff. und zum Begriff der >Aussager siehe Foucault [1969] 1981: 123. 
2016: 90ff.), weshalb diese am ausführlichsten dargelegt und mit „Bedingungen der Verwendungssituation“ (Felder 2016: 90) und textklassen- und textsortenspezifischen Beschreibungsaspekten verbunden wird (vgl. Kap. 5.3.3). Zuvor wird in dieser Arbeit die historisch-topologische Perspektive erläutert (Kap. 5.3.1), da diese direkt an die vorherigen Kapitel zu den gesellschaftspolitischen und fachkulturellen Entstehungsbedingungen und Überlegungen der Korpuszusammenstellung anschließt. In Kap. 5.3.2 werden die Texte zudem hinsichtlich ihrer medialen und konzeptionellen Seite charakterisiert.

Das skizzierte Analysegerüst soll dazu dienen, die Texte der Teilkorpora gewissermaßen ,von oben“ „,vom Standpunkt der Gesamtsprache“ (Felder 2016: 43) und vom Gesamtkorpus aus zu bestimmen (top down). In den Analysen in Großkapitel 6 wird es schließlich darum gehen, anhand von Spuren auf der Sprachoberfläche herauszuarbeiten, welche sprachlichen und sozio-kulturellen Praktiken in welchen konkreten Gebrauchszusammenhängen vorkommen, die zur diskursiven Entstehung einer Definition des Phänomens/Konzeptes `BURNouT` beitragen (bottom up).

\subsubsection{Raumzeitliche Perspektive}

Die Untersuchung startet mit Texten seit Mitte der 1970er Jahre. Für diesen Beginn spricht zum einen, dass Ende der 1960er und im Verlauf der 1970er Jahre Texte erscheinen, die wiederholt und an exponierter Stelle im Burnout-Diskurs von späteren Autorinnen und Autoren aufgegriffen werden ${ }^{291}$, wodurch der Burnout-Diskurs bereits sehr früh eine von den Diskursakteuren selbst produzierte Geschichte erhält (siehe dazu Kap. 6.1.1). ${ }^{292}$ Zum anderen gibt es Indizien dafür, dass insbeson-

291 So wird z. B. "the staff 'burn out' phenomenon" in Bezug auf Mitarbeiter/innen eines Strafvollzugsprogramm von H.B. Bradley in einem Aufsatz mit dem Titel "Community-based Treatment for Young Adult Offenders" (Bradley 1969: 366, im QV unter 8.1.5) erwähnt; Christina Maslach und Susan Jackson berichten in einem Aufsatz aus dem Jahr 1984, dass es 1977 das erste Mal auf einem Kongress der American Psychological Association (APA) ein Symposium zu "burnout" gab, an dem "Freudenberger, Pines, Maslach, and others described their ideas and research findings” (Maslach/Jackson 1984: 137). Karger nennt Freudenberger in seinem Artikel „Burnout as Alienation“ aus dem Jahr 1981 "one of the early writers to identify burnout” (Karger 1981: 271, im QV unter 8.1.5). Zudem wird auch in späterer Fachliteratur zum Thema „Burnout“ Herbert Freudenbergers Artikel "Staff Burn-Out” aus dem Jahr 1974 als Begründungstext und Freudenberger als Begründer zitiert ("originator of the burnout syndrome”, Schaufeli/Enzmann 1998: 2, siehe im QV 8.1.5).

292 Dies wird noch dadurch verstärkt, dass Überblickstexte in der Darstellung der bisherigen Forschung eine Phaseneinteilung vornehmen. Burisch nennt die frühe Phase mit Fallgeschich- 
dere die Verwendung der substantivierten Verben Burn-out/Burnout/Ausgebranntsein/das Ausbrennen zur Bezeichnung physischer und psychischer Erschöpfung in diesem Zeitraum als neu empfunden wurden (vgl. dazu Kap. 6.1.2).

Sprachgeschichtlich lassen sich die Texte des deutschsprachigen fachlichen als auch öffentlichen Korpus der Sprachstufe des ,jüngsten Neuhochdeutschen“ (Bär 2000: 10) zuordnen. Im Folgenden seien linguistische Beobachtungen zu dieser Sprachstufe, die sich in Texten des Untersuchungskorpus widerspiegeln, erläutert. Für die sprachgeschichtliche Einordnung der englischsprachigen Texte sei auf Überblickswerke von Bauer (1994) und Mair (2008) zur Entwicklung der englischen Sprache im 20. Jahrhundert verwiesen. Auf eine gesonderte detaillierte sprachgeschichtliche Analyse des englischen Korpus wird verzichtet, da der Analyseschwerpunkt dieser Arbeit auf deutschsprachigen Texten liegt und einige der Entwicklungstendenzen, die im Folgenden für die Entwicklung im Deutschen genannt werden, auch für den englischen Sprachraum zutreffen.

Als „kulturhistorische Tatsachenkomplexe“, die die Entwicklung der deutschen Gegenwartssprache „nach dem 2. Weltkrieg“ beeinflusst haben, nennt Jochen Bär:

die Entwicklung der Massenmedien einschließlich der neuen Medien, die kommerzielle und kommunikative Globalisierung und die europäische Integration.

(Bär 2000: 12f.)

Eine für die vorliegende Arbeit bedeutsame Entwicklung ist zum Beispiel, dass in der zweiten Hälfte des 20. Jahrhunderts der Anteil der Fachkommunikation an der Gesamtkommunikation zunimmt, wodurch die Fachsprachen - verstärkt durch die Massenmedien und Neuen Medien - einen größeren Einfluss auf die Gemein- bzw. Alltagssprache ${ }^{293}$ erhalten (vgl. Hoffmann 2000: 1994; Bär 2013294). Damit zusammenhängend kommt es zu einem „Zuwachs an Fachwortschatz, zunächst in der fachinternen und interfachlichen, dann aber auch in der fachexternen Kommunikation“ (Hoffmann 2000: 1994).

Im Bereich der fachlichen Terminologisierungsarbeit konstatiert Hoffmann für diesen Zeitraum des Weiteren eine „Tendenz zur Internationalisie-

ten und Selbsterfahrungsberichten (darunter Freudenberger 1974) die „feuilletonistische Phase“, mit dem Maslach Burnout Inventory habe dann die „simplistisch-empirische Phase“ eingesetzt (Burisch ${ }^{5}$ 2014: 238); siehe im QV 8.1.5.

293 Zur Diskussion der Begriffe >Alltagssprache«, >Umgangssprache`, >Gemeinsprache` etc. siehe Steger (1991: 58ff.) sowie Felder (2016: 91ff.).

294 „Eine kurze Geschichte der deutschen Sprache“ hat Bär (2013) online frei zugänglich veröffentlicht unter: http://www.baer-linguistik.de/sprachgeschichte/deutsch03.htm, zuletzt eingesehen am 26.11.2019. 
rung und Standardisierung (Normung)“(ebd.: 1995), welche mit den im obigen Zitat von Bär (2000) anklingenden Tatsachenkomplexen der „kommerzielle[n] und kommunikative[n] Globalisierung “ und ,europäische[n] Integration“ zusammenfällt. Im Fach Psychologie und in den Texten des Untersuchungskorpus finden sich viele Beispiele für Fachausdrücke, die aus dem Englischen entlehnt wurden, oder es sind sowohl die englischen als auch die deutschen Fachausdrücke nebeneinander gebräuchlich: Die Entlehnung Burnout steht in einer Reihe mit Ausdrücken wie Stress, Bias, Work-Life-Balance oder Coping. Dabei habe die „Anpassung (Assimilation) fachsprachlicher Lehnwörter an das phonematische, graphematische und morphologische System der entlehnten Sprache“ in den letzten Jahrzehnten abgenommen (Hoffmann 2000: 1994), wobei die im Rechtschreibeduden empfohlene Großschreibung bei den genannten Beispielen ${ }^{295}$ darauf hinweist, dass diese Fachwörter in diesem Punkt assimiliert wurden.

Mit den Fachwortschätzen, so Hoffmann weiter, hätten auch die Fachtextsorten ${ }^{296}$ zugenommen und „,der enorme Zuwachs der Massenmedien“ habe den Transfer von Fachlexik und -stil in die Gemein- bzw. Alltagssprache „erheblich beschleunigt“ (ebd.: 1996). Ein Prozess, der in den letzten Jahrzehnten auch durch die neuen Medien weiter verstärkt wird. Da die Fächer Psychologie und Medizin existenzielle Lebensbereiche berühren, verwundert es nicht, dass fachsprachliche Begriffe aus diesen Bereichen in „lebenspraktische Begriffe der Alltagssprache“ überführt werden, wie Steger unter anderem am Beispiel des Wortes Stress demonstriert (Steger 1991: 99), und dass die Grenzen zwischen Fach- und Alltagssprache an manchen Stellen fließender werden (vgl. Bär 2013). Dieser Prozess spiegelt sich in alltagssemantischen Vorstellungen der Medizin und Psychologie wider, die jeweils in Wechselwirkungen mit den Fach- und Vermittlungssemantiken stehen. Es kommt also wechselseitig zu einer „Überführung von alltagssprachlichen Ausdrücken und Begriffen in die Fachsprache“ und umgekehrt von Fachsemantiken in die Alltagssprache. In der Alltagskommunikation verwendete Ausdrücke wie Erkältung (vgl. Steger 1991: 64-73), Belastung oder Ermüdung und Erschöpfung werden insbesondere in der Anwendungspraxis zwischen Laie und Experte verwendet. Im Austausch zwischen Fachleuten werden sie allerdings terminologisiert und damit fachsemantisch gefasst. Umgekehrt

295 Siehe auf der Plattform www.duden.de die Lemmata Burn-out/Burnout, Stress, Bias und Coping, zuletzt eingesehen am 26.11.2019.

296 Für die Fachtextsorten konstatiert Hoffmann eine „strengere Formalisierung und z. T. eine starke Unifizierung“. Die Ursachen dafür lägen ,in den Vorgaben der Verlage und Zeitschriftenredaktionen, aber auch in den Anforderungen der Information und Dokumentation“ (Hoffmann 2000: 1995). 
wird eine beträchtliche Anzahl psychologischer und medizinischer Termini in der Alltagskommunikation verwendet. „Begriffe wie Aggression, Depression, Frustration und Verdrängung“ (Steinig 1981: 422) sind aus alltagsweltlichen Gesprächen nicht mehr wegzudenken. ${ }^{297}$

In Bezug auf das Stichwort Internationalisierung seien in diesem Kontext noch die Diskussionen um die Wissenschaftssprachen Deutsch und Englisch und Entwicklungstendenzen der Angleichung der Wissenschaftsstile und Textsorten im Laufe des 20. Jahrhunderts erwähnt. Insbesondere die Vorgaben für Original- oder Übersichtsarbeiten in Fachzeitschriften haben internationale Gültigkeit erlangt (vgl. Kap. 5.3.3.2). In der Psychologie und Medizin zeigen sich, wie in Kap. 5.2. dargelegt, deutliche Tendenzen der Hinwendung zur Publikationssprache Englisch. Für die Anwendungsfächer und Fortbildungsbereiche wiederum ergeben Studien, dass nach wie vor viele Artikel auf Deutsch publiziert und auch rezipiert werden (vgl. Baethge 2008; Schui/Krampen 2016).

\subsubsection{Perspektive der Medialität und Konzeptionalität}

Die Perspektive der Medialität fragt danach, in welcher Weise „jeder Gedanke, der geäußert und damit auch in irgendeiner Weise materialisiert werden muss, von der jeweiligen medialen Erscheinungsform abhängig ist und damit medial geprägt wird“ (Burger/Luginbühl 42014: 3). Medien werden in diesem Zusammenhang verstanden als

„Operatoren“ [...], welche die vermittelten Inhalte nicht nur prägen, sondern immer auch „zugleich mit hervorbringen (ebd.).“

(Burger/Luginbühl ${ }^{4} 2014:$ 3, Fußnote im Zitat nach „ebd.“ entfernt)

297 Wobei diese Begriffe häufig selbst aus anderen Fachbereichen in die psychologische Terminologie übernommen wurden, wie zum Beispiel Aggression. Diese Bezeichnung wurde zunächst im Bereich der internationalen Politik und des Militärs verwendet (vgl. den Eintrag zu Aggression im „Deutschen Fremdwörterbuch“ des Leibniz-Instituts für Deutsche Sprache, abrufbar unter: http://www.owid.de/artikel/405255, zuletzt eingesehen am 26.11.2019); oder der Ausdruck Depression, der zunächst in naturwissenschaftlichen Bereichen und erst seit Anfang des 19. Jahrhunderts als Fachausdruck der Psychologie/Psychiatrie nachgewiesen wurde (siehe den Eintrag im „Deutschen Fremdwörterbuch“ zu Depression (abrufbar unter: http://www.owid.de/ artikel/405956, zuletzt eingesehen am 26.11.2019). Britt Marie Schuster beschreibt die Verwendung des Ausdrucks Depression beim Psychiater Wilhelm Griesinger Mitte des 19. Jahrhunderts, siehe Schuster (2010: 305ff.). 
Es geht ferner um die Frage, wie diese „Formen der Vermitteltheit“ wahrgenommen und welche Erwartungen an sie gestellt werden (Spitzmüller/Warnke 2011: 184).

Das Untersuchungskorpus setzt sich aus Printtexten (bei den Pressetexten auch aus Hypertexten von Online-Massenmedien) und Texten, die im Internet veröffentlicht werden, zusammen. Im fachexternen Korpus findet man neben schriftlichen Texten auch Fotografien, seltener auch Zeichnungen und in Fachtexten neben den schriftlichen Texten, Berechnungsformeln, statistische Grafiken und Tabellen. ${ }^{298}$ Der Analyse-Fokus dieser Arbeit liegt auf Mitteln und Praktiken des Definierens, die sich an der Sprachoberfläche zeigen. Daher wird auf Bilder und Grafiken nicht separat, sondern nur am Rande im Rahmen der Analyse definitorischer Praktiken in den Kapiteln 6.2.4.1 und 6.2.4.2 eingegangen. Die Perspektive der in der Medialität mitzudenkenden Materialität entspricht in Peirce Zeichen-Modell dem Blickwinkel des Zeichenträgers (sign), welcher allerdings wechselseitig mit Zeichenobjekt (object) und Zeicheninterpretant (interpretant) verbunden ist (vgl. Kap. 2.2.2 dieser Arbeit).

Eng verbunden mit dem Kriterium medialer Schriftlichkeit ist die Frage, welche „Kommunikationsformen“ diese Medien ermöglichen (Dürscheid 2011: 94ff.; Schneider/Stöckl 2011: 22ff.) und für welche kommunikativen und situativen Anforderungen sie sich - von konzeptioneller Seite der Sprachverwendung aus betrachtet - prototypisch im Sinne einer ,zuerst geplante[n] Übermittlungssituation“ eignen (Felder 2016: 28 mit Bezug auf Söll ${ }^{3} 1985$ ).

Die Texte des Untersuchungskorpus sind überwiegend monologisch, in gedruckten Interviews auch dialogisch ${ }^{299}$ oder in einigen Textabschnitten bisweilen fingiert dialogisch konzipiert ${ }^{300}$. Sie wirken überwiegend vorbereitet, sind thematisch fixiert, öffentlich ${ }^{301}$, geprägt von örtlicher und zeitlicher Distanz/Diskontinuität und beinahe durchgehend mit vorzeitiger Zeitreferenz. Letzteres hängt auch mit dem Untersuchungsgegenstand zusammen: Dem Begriff der >Krankheit ist das Attribut der 'Vorzeitigkeit' immanent. Auf der Achse „konzeptionelle Schriftlichkeit/Mündlichkeit“ lassen sich die Texte dadurch deutlich dem Pol der konzeptionellen Schriftlichkeit zuordnen.

298 Beiträge gesprochener Sprache (z. B. Videos oder Talk-Shows) sind nicht Teil des Korpus. 299 Die Dialoge, die in der Presse als gedruckte Texte erscheinen, haben die Besonderheit, dass sie zwar ursprünglich auf ein Gespräch (face-to-face oder telefonisch) zurückgehen, dass viele Merkmale des Mündlichen jedoch (wie Versprecher, unnötige Wiederholungen etc.) für den Druck entfernt werden (Burger/Luginbühl ${ }^{4} 2014:$ 176f.).

300 Fingierte Dialoge finden sich wiederholt in ratgebenden Texten oder im Kontext von Fragebögen zum Thema „Burnout“, so z. B. im Beitrag „Total ausgebrannt - und jetzt?“ der AU 12/1997-A: 20: „Sind Sie reif für die Insel? Diese Alarmzeichen sollten sie beachten: Sie sind ständig müde [...]“ (= AU 1997). Vgl. auch PH 1/2016: 25: „Sind Sie Burnout-gefährdet? Bitte beantworten Sie alle Fragen [...]““ (= Nelting 2016, im Quellenverzeichnis unter 8.1.7 im Abschnitt „Psychologie Heute“).

301 Vgl. Anmerkung 215 in Kap. 5.1.0. 


\subsubsection{Perspektive der kommunikativen Reichweite der Ausdrücke und funktionalen Reichweite der Inhalte in fachinternen und fachexternen Kontexten}

\subsubsection{Vorbemerkung}

Ausdrucksseitig haben wir es bei den Texten des Korpus beinahe durchweg mit Standardlekt zu tun, sowohl bei den fachlichen als auch bei den vermittlungssemantischen Texten. Die weitaus größte Differenzierung der Teilkorpora ergibt sich durch die Dimension der funktionalen Reichweite der Inhalte. Dies sei an folgendem Beispiel illustriert:

$\mathrm{Zu}$ den Wörtern belasten und Belastung, die in Kap. 5.3.1 als Übernahmebeispiele aus der Alltags- in die Fachsprache angeführt wurden, findet man im Deutschen Wörterbuch der Brüder Grimm u. a. folgende Einträge:

1) sinnlich, den wagen mit steinen, das schif mit waaren belasten; bäume mit früchten belastet; [...]

2) figürlich, ein hohes alter belastet; willkommne aufträge belasten nicht; er ist mit verbrechen belastet; das haus mit schulden belasten; das land mit schweren steuern; ach schon lang hat mir der kummer mein leben belastet. ${ }^{302}$

Klopstock Mess. 7, 484

Der Eintrag zeigt, dass der sogenannte „figürliche“ Gebrauch seit Jahrhunderten im Sprachgebrauch fest verankert ist und dass der Vorgang bzw. damit einhergehende Endzustand überwiegend negativ konnotiert ist. ${ }^{303}$ Zum fachsprachlichen Gebrauch der Normung (DIN EN ISO 10075-1) hingegen weist Nachreiner darauf hin, dass „Belastung wie auch Beanspruchung, [...] als neutral definierte Konzepte $\mathrm{zu}$ verstehen sind und daher per se nicht etwas grundsätzlich zu Vermeidendes darstellen, wie das der umgangssprachliche Gebrauch der Begriffe nahelegt“ (Nachreiner 2012: 10). Dieses Beispiel verdeutlicht, dass das Wort Belastung im fachsprachlichen Kontext auf Fachzusammenhänge referiert, die nur eingeweihten Fachleuten vertraut sind. Dies schränkt die kommunikative Funktionsreichweite der Semantik ein, ohne dass dies einem Laien auf den ersten Blick bewusstwerden muss.

Nachfolgend seien die Texte der Teilkorpora daher hinsichtlich ihrer Wissensvoraussetzungen, Produzenten- und Adressatengruppen sowie ihrer damit

302 Siehe den Eintrag in der Online-Ausgabe des Deutschen Wörterbuchs von Jacob und Wilhelm Grimm, unter: http://www.woerterbuchnetz.de/DWB?lemma=belasten, zuletzt eingesehen am 26.11.2019.

303 Vgl. dazu auch die Einträge zu belasten und Belastung auf duden.online unter: http://www. duden.de/rechtschreibung/belasten, http://www.duden.de/rechtschreibung/Belastung, zuletzt eingesehen am 26.11.2019. 
verbundenen funktional zweckhaften Leistung und Kommunikationsbereiche noch eingehender beurteilt. Damit zusammenhängend werden die besonders einschlägigen Textsorten der Teilkorpora und alle weiteren damit verbundenen situativen und kontextuellen Faktoren eruiert, um eine pragmasemantische Charakterisierung der Erscheinungsformen der Texte zu ermöglichen.

\subsubsection{Texte in Fachlexika, Handbüchern, Lehrbüchern, gedruckten Fachenzyklopädien und Kompendien}

Texte sowohl in Fachlexika als auch in Hand- und Lehrbüchern haben die Aufgabe, ihren Leserinnen und Lesern einen komprimierten, systematischen Überblick über Grundbegriffe und Themen eines Fachs zu bieten. Die Adressatengruppen sind Studierende und Wissenschaftler/innen der einschlägigen Fächer, aber auch „praktizierende Fachpersonen“ und bei den Fachlexika auch „interessierte Laien“. ${ }^{304}$ Zusammenhängend mit diesen Zielgruppen wird auf eine mittlere bis hohe Reichweite des Ausdrucks- und Inhaltssystems geachtet, wobei sich aber auch terminologische Taxonomien hinter den Ausdrücken verbergen. Letztere können in der Regel innerhalb der Werke über Glossare oder Verweise nachvollzogen werden. Durch diese Verweise entsteht ein Netz zwischen den Lexikoneinträgen, ${ }^{305}$ das Hypertexten ähnelt, die man „nicht-linear durcharbeitet“: Die Verweise laden wie elektronische Verknüpfungen zum „Herumspringen“ ein (Adamzik 2002: 178), und sie können ,in beliebiger (bzw. von den Links ermöglichter) Reihenfolge“ gelesen werden (Burger/Luginbühl 42014: 458). Die einzelnen Stichworteinträge folgen allerdings dennoch einem linearen Textaufbau und die Verweise sind sparsamer eingesetzt als in hypertextuellen Beiträgen der Onlineenzyklopädie Wikipedia (siehe Kap. 5.3.3.5).

Diese Texte entstammen dem Kommunikationsbereich der fachlich-vermittelnden Kommunikation bzw. Kommunikation im Bildungsbereich. Die Anforderungen an das Medium Lexikon im Besonderen, aber auch an das Handbuch und Lehrbuch, einen breit gefächerten Überblick auf überschaubarem Raum zu ermöglichen, bewirken eine äußerst komprimierte, ökonomische (mit Nominalisierun-

304 Vgl. in der Online-Ausgabe von „Dorsch: Lexikon der Psychologie“ die dort angegebenen Adressatengruppen: https://portal.hogrefe.com/dorsch/de/ueber-den-dorsch/, zuletzt eingesehen am 28.11.2019; siehe auch Wiese (2000: 715) zum Adressatenkreis des Nachschlagewerks „Pschyrembel. Klinisches Wörterbuch“.

305 Burkhard Schaeder diskutiert in diesem Zusammenhang die Frage, ob und inwiefern man davon sprechen kann, dass die einzelnen Wörterbuchartikel als „Teiltexte“ eines Textes, nämlich des „fachlichen Sachwörterbuchs“ als Ganzem angesehen werden können (siehe dazu Schaeder 1996: 116ff.). 
gen arbeitende) und unpersönliche Schreibweise. ${ }^{306}$ Diese dem Platz geschuldete Eigenschaft verringert wiederum die Verständlichkeit und inhaltliche Reichweite dieser Texte. Die einzelnen Artikel in den Lehrbüchern und Fachlexika wirken insgesamt stärker standardisiert als in den Handbüchern und Fachenzyklopädien, die den Autorinnen und Autoren mehr Freiheiten einräumen.

Lexika und Handbücher dienen häufig zum Einstieg in ein Thema auch unter Fachleuten. Sie werden in der Regel von einer „Vielzahl von Spezialisten“ (Wiese 2000: 715) verfasst und besitzen dadurch Autorität. Das Verhältnis von Produzenten (mit „Autoritätszügen von Seiten der Schreibenden“) und Rezipienten ist „tendenziell asymmetrisch“ (Schuster 2010: 284). Des Weiteren präsentieren insbesondere Lexika und Lehrbücher ,in der Regel einen relativ aktuellen Stand“ (Wiese 2000: 715). Um die Entwicklung der Erkenntnis zum Phänomen BuRNOUT nachvollziehen zu können, wurden von den untersuchten Fachlexika und Lehrbüchern mehrere Auflagen in das Untersuchungskorpus aufgenommen (vgl. hierzu Kap. 5.1.2 und 6.2.2.1 dieser Arbeit und im QV 8.1.2).

\subsubsection{Texte in Fachzeitschriften, Fachbüchern und Sammelbänden}

Die Texte dieses Abschnitts richten sich alle an Wissenschaftler/innen (darunter teilweise auch Studierende) und (praktizierende) Fachleute des eigenen psychologisch-medizinischen Spezialfachs oder sachverwandter Fächer. Die Textproduzentinnen und -produzenten sind ebenfalls Fachleute, die in fachkulturelle, literale und soziokulturelle Praktiken eingebunden sind. Insbesondere bei den sogenannten „Originalarbeiten“, die in Fachzeitschriften erscheinen und der Publikation von neuen Forschungsergebnissen unter der Berücksichtigung bisheriger Forschung dienen, wird tiefergehendes Fach- und Methodenwissen vorausgesetzt. Dies zeigt sich daran, dass statistische Kennzahlen, Modelle oder Fachtermini ohne Erklärung angeführt werden, was die inhaltliche Reichweite deutlich einschränkt. ${ }^{307}$ Diese Texte sind in Bezug auf die Gliederung „stark konventionalisiert“ (Busch-Lauer 2001: 51). Sie basieren auf der IMRaD ${ }^{308}$-Struktur nach anglo-amerikanischem Vorbild (vgl. ebd.: 54; 64), die in vielen Fachzeit-

306 Diese stilistischen Merkmale beschreibt Schuster schon für die Entwicklung psychiatrischer Lehrbücher am Ende des 19. und zu Beginn des 20. Jahrhunderts (Schuster 2010: 334ff.). Sie prägt dazu den prägnanten Satz: „Der Kranke verschwindet und wird Träger von Krankheitszeichen“ (ebd.: 335).

307 Beispiele aus dem Korpus sind z. B. Michel/Stegmaier/Meiser/Sonntag (2009) in der ZfAO oder Bermejo/Fritz (1993) in der PPmP (siehe QV 8.1.4).

308 Die Abkürzung steht für Introduction, Methods, Results and Discussion. 
schriften unter den Hinweisen für Autorinnen und Autoren teilweise per Checkliste vorgeschrieben ist. ${ }^{309}$

Die Textsorte Originalarbeit hat in der Regel folgenden Aufbau: Kopf - deutsche und englische Zusammenfassung - deutsche und englische Schlüsselwörter - Einleitung - Methodik Ergebnisse - Diskussion - Schlußfolgerungen (fakultativ) - Danksagung (fakultativ) - Literaturverzeichnis. Der Kopf des Beitrags besteht aus folgenden Angaben: Überschrift (teilweise mit Untertitel), Name des Autors bzw. Namen der Autoren ${ }^{310}$, Bezeichnung und Ort der Klinik/ des Instituts, aus dem die Arbeit stammt, einschließlich der Nennung des Leiters der Institution. ${ }^{311}$

(Wiese 2000: 710)

Ebenfalls fachsemantisch komplex sind Fachtexte, in denen Messinstrumente in ihrer Entwicklung und Anwendung vorgestellt werden. Denn sie richten sich primär an Forschende und Expertinnen und Experten mit dem Ziel, diese von der Brauchbarkeit des Messverfahrens für Anschlussstudien und für die Diagnostik zu überzeugen, und sie leiten an, wie diese Instrumente anzuwenden sind.

Eine neben den Originalarbeiten ebenfalls häufige Textsorte im fachlichen Zeitschriftenkorpus stellen die Übersichtsarbeiten/Reviews dar, die bisweilen als Leitartikel erscheinen. ${ }^{312}$

Übersichten sind Sekundärtexte. Sie werden vielfach auf Anforderung der Schriftleitung von renommierten Autoren verfaßt. Übersichtsarbeiten bieten den aktuellen Stand zu einem praxisrelevanten Thema mit dem Ziel der Fortbildung [...].

(Wiese 2000: 712)

Von der Struktur sind sie in ihren Mittelteilen teilweise freier als Originalarbeiten, die meisten weisen aber ebenfalls feste Bestandteile auf: Nach dem (deutschen und englischen) Titel folgt eine deutsche (und englische) Zusammenfassung, Schlüsselwörter, Einleitung, Hauptteil mit themenabhängigen Zwischenüberschriften, Resümee/Ausblick und Literaturverzeichnis. Übersichtsarbeiten aus dem Bereich der Medizin bzw. Psychiatrie und Psychotherapie enthalten häufig, ähnlich zu Lehrbuchartikeln über Krankheiten, Abschnitte zu Definition, Ätiolo-

309 Vgl. zum Beispiel den Leitfaden für Autoren der Zeitschrift „Der Nervenarzt“ unter: http:// static.springer.com/sgw/documents/69332/application/pdf/Leitfaden_Originalien.pdf, (zuletzt eingesehen am 28.11.2019).

310 Die Mehrautorenschaft ist ebenfalls charakteristisch für medizinische Originalarbeiten im Vergleich zu Forschungsarbeiten aus dem linguistischen Bereich (vgl. Busch-Lauer 2001: 64).

311 Busch-Lauer arbeitet einige interlinguale Aspekte zwischen deutschen und englischsprachigen/internationalen Originalarbeiten der Medizin heraus (siehe Busch-Lauer 2001, zu „Abstracts“ siehe Busch-Lauer 2007).

312 Beispiele aus dem Korpus: Berger/Schneller/Meier (2012) in „Der Nervenarzt“ oder Kissling/ Mendel/Förstl (2014) in der DMW (siehe QV 8.1.4). 
gie und Pathogenese, Prävalenz/Epidemiologie, Diagnostik und Differentialdiagnostik, Prävention, Therapie (vgl. dazu Wiese 2000: 713f.). In einigen Texten wird die Übersicht mit konkreten Fallbeispielen und Praxisberichten verbunden. ${ }^{313}$ Neben dem Ziel der Fortbildung über neue Sachinformationen geben die meisten Übersichtsartikel im abschließenden Textabschnitt Handlungsempfehlungen. Ein Teil der Arbeiten konzentriert sich in diesen Abschnitten auf Vorschläge für konkrete Therapie- und Prophylaxeansätze ${ }^{314}$, wohingegen ein anderer Teil der Arbeiten die Burnout-Forschung und den (fachlichen und öffentlichen) Umgang mit diesem Thema insgesamt (kritisch) beleuchtet und Vorschläge für den zukünftigen Umgang mit diesem Thema unterbreitet. ${ }^{315}$

Neben diesen Textsorten veröffentlichen einige Zeitschriften auch Berichte über einzelne Studien oder Umfragen ${ }^{316}$ und „,ratgebende Aufklärungstexte“317 über BURNOUT in medizinischen Berufen, die teilweise in Rubriken wie „Themen der Zeit“, „Aktuell“ oder „Arbeitswelt“ erscheinen. Des Weiteren finden sich in einigen Fachzeitschriften wie dem „Deutschen Ärzteblatt“ oder der „Deutschen medizinischen Wochenschrift“ auch Texte, die den Textsorten Nachricht/Meldung ${ }^{318}$, Kurzbeitrag bzw. Bericht über die gesundheitspolitische Lage und Studienergebnisse $^{319}$, Leserbriefe ${ }^{320}$ und Buchrezensionen ${ }^{321}$ zugeordnet werden können.

Die Textsorte Kasuistik oder Fallbeschreibung schließlich, der „ein spezifischer Stellenwert für den ärztlichen Wissens- und Erfahrungserwerb beigemessen“ wird (Wiese 2000: 711), findet sich in geringerem Ausmaß im Korpus als erwartet. Matthias Burisch fordert sogar einen Zuwachs an Kasuistiken für den

313 Beispiele dazu aus dem Korpus: Weimer/Kraus (2011); Kissling/Mendel/Förstl (2014); siehe im QV unter 8.1.4.

314 Z. B. Bauer/Häfner/Kächele et al. (2003) in PPmP (siehe im QV unter 8.1.4).

315 Z. B. Kaschka/Korczak/Broich (2011) im Dt. Ärztebl. oder Kapfhammer (2012) in der Zeitschrift „Der Internist“ (siehe im QV unter 8.1.4). Ein satirisch-kritischer Beitrag ist der mit „Glosse“ überschriebene Beitrag „Burn-out: Innenansichten“ im Dt. Ärztebl. (= Voß 2014, im QV unter 8.1.4).

316 Zum Beispiel Dieke/Schmidt/Katzer (2002) oder Will (2014) im „Dt. Ärzteblatt“ oder Weiß (2013) in der Zeitschrift PPmP (siehe im QV unter 8.1.4).

317 Die Textsorte „ratgebender Aufklärungstext“ wird in Kapitel 5.3.3.4 eingehend beschrieben. Beispiele aus dem Korpus sind: Madel (2002) im Dt. Ärztebl. oder Bergner (2016) in der DMW (siehe im QV unter 8.1.4).

318 Z. B. Richter-Kuhlmann (2012), dapd (2011) und me/hil/aerzteblatt.de (2018), alle im Dt. Ärztebl., siehe im QV unter 8.1.4.

319 Z. B. Bühring (2001; 2005; 2012); Hillienhof (2012) und Flintrop/Rieser (2014), alle im Dt. Ärztebl. und Bossenmeyer (2012, DMW), alle Belege im QV unter 8.1.4.

320 Siehe im QV in Kap. 8.1.4 die Beiträge, die im Abschnitt „Dt. Ärztebl“ mit „Leserbrief“ ausgezeichnet sind.

321 Z. B. Tölle (2001); Klinkhammer (2014), beide im Dt. Ärztebl. (im QV unter 8.1.4). 
Fortschritt der Burnout-Forschung (Burisch ${ }^{5} 2014$ : 239, im QV unter 8.1.5). Dies ist erstaunlich, da die sogenannten Initialtexte von Freudenberger Fallskizzen und (Selbst-)Beobachtungen aus der Praxis enthalten (Freudenberger 1974; Freudenberger/Richelson 1980a/1980b, im QV unter 8.1.5). Diese seien jedoch, wie Burisch in Bezug darauf schreibt, ohne „greifbare Ätiologie“ und übergreifende Erklärungshypothese und dadurch zu „oberflächlich angelegt“ gewesen (Burisch 52014: 239). Ähnliche Komponenten werden auch in den Autorenrichtlinien der Deutschen medizinischen Wochenschrift für die Textsorte Kasuistik gefordert. Die Kasuistik soll „neue Erkenntnisse zum klinischen Bild, zur Ätiologie, (Differenzial-)Diagnostik oder Therapie“ einer bekannten Krankheit oder neuen ungewöhnlichen Erkrankung etc. liefern. ${ }^{322}$ Drei ,mustergültige‘ Kasuistiken und einige weniger strenge Fallbeispiele sind dennoch im fachlichen Textkorpus vertreten. ${ }^{323}$

Die Grenzen zwischen fachinternen (intra- und interfachlichen) Texten und Hybridformen, die sich an Expertinnen/Experten, Praktizierende in psychosozialen Berufen (z. B. Krankenpflegepersonal) und relative Laien ${ }^{324}$ richten, sind hinsichtlich ihres Fachlichkeitsgrades und damit auch der Reichweite der Inhalte fließend.

\subsubsection{Fachexterne Texte in der Presse}

Die in dieser Arbeit untersuchten schriftlichen Texte des fachexternen öffentlichen Diskursstrangs, die in überregionalen Tages- und Wochenzeitungen und deren Onlineportalen erscheinen (sowohl als Druck- und Onlineangebote), zeigen folgende charakteristische Merkmale von journalistischen Medientexten $^{325}$ : Die Kommunikatoren bzw. Produzenten der Medientexte sind überwiegend (Wissenschafts-)Journalistinnen und Journalisten, die ihre Texte in periodisch erscheinenden, der Aktualität verpflichteten Organen veröffentlichen und in redaktionelle Abläufe und ressortspezifische (online)journalistische, mas-

322 Vgl. die Autorenrichtlinie der „Deutschen medizinischen Wochenschrift“, abrufbar unter: https://www.thieme.de/statics/dokumente/thieme/final/de/dokumente/zw_dmw/DMW_Autorenrichtlinien_Kasuistik.pdf, (zuletzt eingesehen am 28.11.2019).

323 Z. B. Wilms (2009) in PiD; Weimer/Kraus (2011) in „Der Psychotherapeut“; ein kurzer Fallbericht bei Kissling/Mendl/Förstl (2014) in der DMW; alle Belege im QV unter 8.1.4; Die Grenzen zwischen fachwissenschaftlichen Kasuistiken und kurzen, im fachlichen Sinn unvollständigen Fallbeschreibungen in Monografien und vermittlungssemantischen Fachbüchern sind fließend. 324 Zum Begriff des relativen Laien vgl. Liebert (1996: 93ff.).

325 Unter Medientexten werden im Anschluss an Burger/Luginbühl ( ${ }^{4} 2014:$ 93) „alle Arten von TEXTEN“ verstanden, „die in den Massenmedien angeboten werden“. Im Fokus des medialen Diskursstrangs dieser Arbeit stehen gedruckte journalistische Vermittlungstexte in den Massenmedien und Texte der Online-Plattformen dieser Zeitungen und Magazine. Dabei liegen viele Texte seit den 2000er Jahren sowohl gedruckt als auch online vor. 
senmediale, literale und soziokulturelle Praktiken und Textsortenroutinen eingebunden sind. Sie treten als Vermittlerinstanz auf, lassen aber auch verschiedene fremde Stimmen von Experten- und Laienseite in ihren Texten (in)direkt ${ }^{326}$ $\mathrm{zu}$ Wort kommen (insbesondere Experten/Expertinnen aus dem medizinischen, psychologischen/psychotherapeutischen und pädagogischen Bereich und Individuen, die mit BURNOUT in Berührung gekommen sind). Sie kommunizieren an eine Vielzahl von Rezipienten und Rezipientinnen. Diese sind anonym, heterogen und dispers ${ }^{327}$, auch wenn es auf der Sprachoberfläche Indizien zumindest für den „intendierten Rezipienten“ (Burger/Luginbühl 42014: 11) gibt, d. h. welche Rezipientengruppe vom „Kommunikator explizit oder implizit anvisiert wird“ (Burger/Luginbühl 42014: 11). Schon die Aufzählung von Berufsgruppen, in denen Personen besonders häufig ausbrennen sollen (z. B. Profi-Sportler ${ }^{328}$ und die ,weißen Berufe ${ }^{\text {“329 }}$ ), oder die Nennung von Charaktereigenschaften typischer ,Betroffener (,den Zwang jede Aufgabe perfekt zu erledigen““330) skizzieren im Kontext der Wissensvermittlung und Aufklärung aus der Sicht des Textproduzenten eine Leserschaft, für die dieser Text besondere Relevanz besitzen könnte (Burger/Luginbühl 42014: 11). Weiterführende Beobachtungen, auf welche Weise Textproduzenten sich im medialen Burnout-Diskurs einen „Kreis von intendierten Rezipienten definieren“ (ebd.: 12), werden in Kap. 6.2.4.1 im Lichte einer Praxis des Definierens differenziert werden.

326 In Gastbeiträgen und in Interviews kommen Expertinnen u. Experten direkt zu Wort. In Interviews gibt allerdings der/die Interviewende die Blickwinkel auf das Thema durch seine Fragen vor. Des Weiteren werden Expertenstimmen in Medientexten durch Zitate ,hörbar‘. Der/die Journalist/-in macht die fremden Denkinhalte wörtlich oder paraphrasiert sichtbar oder deutet sie teilweise nur an, wenn er/sie die „inhaltliche Sachkenntnis“ (Köller 2004: 688) zum Erkennen des Zitats bei der Leserschaft voraussetzt.

327 D. h., dass „zwischen den Individuen, die das Publikum bilden, keine Gemeinsamkeit bestehen“ müssen (Burger ${ }^{4}$ 2014/Luginbühl: 8). Man kann jedoch einerseits vermuten, dass eine Gruppe Lesender, die Interesse am Thema „Burnout“ hat, von diesem Thema im privaten oder auch beruflichen Umfeld tangiert ist.

328 Vgl. zahlreiche Berichte schon Ende der 1980er Jahre über ausgebrannte Tennisspielerinnen und später Sportler/innen aus dem Fußball, Handball, Skispringen, etc. (z. B. DER SPIEGEL (1989: 222-224): Tennis: Das Letzte abverlangt; SPIEGEL ONLINE (2004): Sven Hannawald: Kein Comeback in naher Zukunft; Wenzel (2012): Fußballerin mit Burnout. Zurück in die Spur. Auf: FAZ.NET, 02.03.2012; alle Belege im QV unter 8.1.6.

329 So zusammenfassend für Ärzte/Ärztinnen, Krankenpfleger/innen, Altenpfleger/innen, etc. in einem FAZ-Artikel vom 29.02.1992: „Ausgebrannt im Teufelskreis übersteigerter Berufsansprüche“ (= Gross 1992: 41).

330 So zum Beispiel in einem Artikel auf FAZ.NET vom 08.03.2010: „Burnout-Syndrom. Erschöpft, ausgebrannt, arbeitsmüde“ (= Meck 2010); im QV unter 8.1.6. 
Pressetexte, die BuRnout als Hauptthema oder als wichtiges Nebenthema des Textes behandeln, kann man folgenden Textsorten bzw. Textsortenmischungen zuordnen: ${ }^{331}$

- Gedruckte und Hypertexte nach dem Muster des Magazinberichts und Features, die insbesondere durch ihren personalisierten szenischen Beginn und Ausstieg (oft in der Form eines Bogenschlagens, vgl. dazu auch Liebert 2002: 269), viele Zitate, Bilder und Grafiken und den Wechsel zwischen deskriptiven/explikativen und narrativen Textpassagen auffallen; ${ }^{332}$

- Meldungen/Berichte/Problemdarstellungen ${ }^{333}$ und Porträts über (prominente) Fälle ${ }^{334}$, zur Verbreitung des Burnout-Phänomens in der Bevölkerung/in Branchen auf der Basis von statistischen Erhebungen, Studien oder Umfragen ${ }^{335}, \mathrm{zu}$ weiterführenden Studien und Tagungen/Kongressen ${ }^{336}$, Expertenstandpunk-

331 Bei der Einordnung der journalistischen Textsorten orientiert sich diese Arbeit an den Kriterien nach Lüger (21995: 77-151); Wolff (2006:74-234) und Burger/Luginbühl ( ${ }^{4} 2014: 219 \mathrm{ff}$ ).

332 Z. B. der Beitrag „Volk der Erschöpften“ im SPIEGEL 4/2011 (= Dettmer/Shafy/Tietz 2011) oder „Verbrannte Seelen“ in der ZEIT vom 2. Dezember 1999 (= Blech 1999); oder „Müde, antriebslos, zynisch“ in der SZ vom 30.10.2012 (= Haas/Wolff 2012); siehe auch Peikert (2017, FAZ. NET); Maeck (2013) und Zeltner (2011) auf SPIEGEL ONLINE - Karriere Spiegel sowie Wöhrle (2011) auf SPIEGEL ONLINE - UniSPIEGEL; Rudzio (2010a, DIE ZEIT), alle Belege im QV unter 8.1.6.

333 In diesen Texten ist die informierende Funktion gegenüber der kommentierend-wertenden dominant, aber die Texte enthalten natürlich auch ,implizite Wertungen [...] z. B. durch Selektion, Anordnung und Gewichtung in der Sachverhaltsdarstellung “ (Lüger ${ }^{2}$ 1995: 69).

334 Z. B. Nachrichten/Berichte über das „Burn-out-Syndrom“ bei Sven Hannawald, FAZ 3.5.2004, (= dpa 2004b: 38) oder auf SPIEGEL ONLINE am 8.9.2013 (= Ade 2013) oder SPIEGEL ONLINE (2011a); im QV unter 8.1.6.

335 Z. B. die Nachricht über Ergebnisse des DAK-Gesundheitsreports, demzufolge „die Zahl der Menschen mit Depressionen und Burnout weiterhin steigt“ auf SPIEGEL ONLINE (= Hei/AFP/ dpa (2013) und SPIEGEL ONLINE (2011c) und über Burnout-Zahlen in verschiedenen Konzernen auf SPIEGEL ONLINE (= Werle 2012a); siehe auch DER SPIEGEL (2004); Paschen (1995) und Bohsem (2012b) in der SZ und in der FAZ/auf FAZ.NET: AFP/DPA (2010), Dap/Dapd (2012), Schwenn (2012); Mihm (2014); weku (2004), dc./ami (2014), Krohn (2011), Grossarth (2012); alle Belege im QV unter 8.1.6.

336 Z. B. eine Nachricht über eine Studie des Sigmund-Freud-Instituts und der TU Chemnitz in der FAZ (vom 28.3.2012, Nr. 75) unter dem Titel „Soziale Anerkennung verhindert Burn-out“ (= FAZ 2012a: 34); ein Bericht über eine Studie der SRH Hochschule Heidelberg zum Risiko von Führungskräften, psychisch zu erkranken (= FAZ.NET 2015) oder ein Gastbeitrag über eine Studie der Bertelsmann Stiftung gemeinsam mit der „sciencetransfer GmbH in Zürich“ zum Zusammenhang von Führungsstil und Burnout in Unternehmen (= Hollmann/Hannburg 2010, FAZ); Die SZ berichtet am 20.11. 1997 über Diskussionen zum „Burn-out-Syndrom“ auf dem „10. Medizin-Theologie-Symposion der Evangelischen Akademie Tutzing“ (= Pförtner-Hüttner 1997) und am 30.10.2013 über eine TK-Studie zur Stresslage der Nation (= Bruckner 2013); DER SPIEGEL berichtet über einen Kongress (= Frey 2012) und Lehrer-Studien (= Bebber 2008; Leffers 
ten $^{337}$, juristischen Tipps und Gerichtsentscheidungen ${ }^{338}$, zu Vorträgen und Lehrwerken $^{339}$, Problemen in bestimmten Berufssparten ${ }^{340}$ und politischen/ wirtschaftlichen Reaktionen/Maßnahmen ${ }^{341}$ - gedruckt und online;

- Interviews mit Expertinnen/Experten und Personen, die Erfahrungen mit BURNOUT und/oder Begleitfaktoren schildern, - gedruckt und online; ${ }^{342}$

- Erfahrungsberichte aus der Ich-Perspektive mit BuRNouT ${ }^{343}$ und Erfahrungsberichte/Leserbriefe, die die Zuschreibung 'Burnout' kritisieren ${ }^{344}$ - gedruckt und online.

2008); im Beitrag Dpa/svs/tine (2015, FAZ) wird die Studie „Psychische Belastungen und Burnout beim Bildungspersonal“ erwähnt; Hinweis auf eine Tagung in FAZ (2013a); alle Belege im QV unter 8.1.6.

337 Z. B. in DIE ZEIT Nr. 49/2011 (= Albrecht 2011a, im QV unter 8.1.6.)

338 Siehe z.B. die Nachricht über ein Urteil zur Frage, ob die Berufsunfähigkeitsversicherung bei Burnout zahlen muss, in der FAZ vom 3.8.2004, Nr. 178 (= weku 2004: 50), Jur/FAZ (2007) und FAZ (2007a); oder das Urteil im Streit darum, ob eine „Betriebsrätin mit Burnout [...] segeln gehen“ durfte, auf SPIEGEL ONLINE 5.5.2012 (= Leffers 2012); im QV unter 8.1.6.

339 In allen untersuchten Medien findet man kurze Meldungen, die Vorträge zum Thema „Burnout“ ankündigen: z. B. Jff (2011 und 2014, in der FAZ) und FAZ (2011a; 2012c); Die zuordnend-eingrenzende Wirkung von Vortragsankündigungen wird in Kapitel 6.2.4.1 an Belegen der SZ ausführlich beschrieben. In einer Meldung der FAZ wird auf das Lehrbuch von Xaver Baur (siehe im QV unter 8.1.2 unter Groth, (2013)) verwiesen (= FAZ 2013d); Belege im QV unter 8.1.6.

340 Z. B. in der SZ vom 13.1.2000 über die Probleme des Berufsstands der (Alten-)Pfleger/innen. Das „Burnout-Syndrom“ wird als eine Folge „bei vielen Pflegern“ genannt (= Geschuhn 2000, im QV unter 8.1.6).

341 SPIEGEL ONLINE berichtet beispielsweise 2013 über die „Anti-Stress-Verordnung“ und den „Stressreport“, den Ursula von der Leyen (in dieser Zeit Arbeitsministerin) vorstellte (= dpa/ dapd/end 2013). Die SZ berichtet am 2.5.2012 über eine Stellungnahme der Bundesregierung auf eine Anfrage der Linken zu psychischen Erkrankungen am Arbeitsplatz (= SZ/AFP 2012: 19) und am 30.10.2012 über die Forderung SPD-regierter Länder nach konkreten Vorgaben für Arbeitgeber, wie sie „dem Burn-out vorbeugen“ sollten (= Bohsem/Haas 2012: 17); die FAZ berichtet am 30.1.2013 darüber, welche Maßnahmen Firmen zur Burnout-Prävention schon ergreifen und welche weiteren Forderungen es an sie zum Schutz der psychischen Gesundheit der Beschäftigten gibt (= Steinau-Steinrück 2013: 19); alle Belege im QV unter 8.1.6.

342 Siehe zum Beispiel in DIE ZEIT, Nr. 30/2006 vom 20.07.2006 (= Kuhrt 2006); oder das Interview im ZEITmagazin Nr. 39/2014 6. Oktober 2014 mit Miriam Meckel (= Koelbl 2014) und im SPIEGEL (= Beyer/Voigt 2010); oder auf Spiegel Online das Interview mit Ulrich Hegerl am 24.11.2011 (= Kramer 2011) und Gottschalck (2012); siehe auch Freund (2013, FAZ); Schmidt (2014, FAZ), Budras (2013, FAZ) und Astheimer (2014a, FAZ); Dettmer/Tietz (2011, SPIEGEL ONLINE), Hinrichs/ Meyer (2004, SPIEGEL SPECIAL), alle Belege im QV unter 8.1.6.

343 z. B. auf ZEIT Campus 3/2010 (= Schwabe 2010) und einzelne Fallbeispiele aus der IchPerspektive in DIE ZEIT 27/2013 (= Schoener 2013); oder auf SPIEGEL ONLINE am 8.8.2012 (= Abé 2012); oder in der FAS am 13.9.2015 (= Oberhuber 2015: 35); alle Belege im QV unter 8.1.6.

344 Z. B. Brock (2008, FAZ) und ein Leserbrief im SPIEGEL (2010a) zum Interview mit Miriam Meckel im SPIEGEL 10/2010 (= Beyer/Voigt 2010); in DIE ZEIT 18/2012 findet ein ,Betroffener` den 
- Fotostrecken ${ }^{345}$ - online;

- Glossen, kurze Kommentare, Kolumnen und satirische Texte zum Thema „Burnout“ - gedruckt und online; ${ }^{346}$

- Kommentierende und erörternd-essayistische Texte zum Thema - gedruckt und online; 347

- Kritiken/Kommentare und Berichte, die auf Bücher, Medienprodukte, Theaterstücke, Ausstellungen, Musik oder Filme zum Thema bzw. mit Bezug auf das Thema „Burnout“ eingehen - gedruckt und online; ${ }^{348}$

- Fragebogen zum Thema „Burnout“ mit Anleitung zur Selbstauswertung ${ }^{349}$ gedruckt und online.

„Begriff Burn-out“ für seine Geschichte „unpassend“ und spricht von einer „Erschöpfungsdepression“ (= Srikiow 2012).

345 Z. B. auf SPIEGEL ONLINE „Fotostrecke: Was Führungskräfte über das Phänomen Burnout denken“ (= SPIEGEL ONLINE 2012c); oder „Volkskrankheit Burnout: Wie Erschöpfung die Volkswirtschaft schwächt“ (= SPIEGEL ONLINE 2011e); siehe auch SPIEGEL ONLINE (2011b) und (2012 $c$ und $\mathrm{d})$.

346 Zum Beispiel in ZEIT Nr. 28, 2003 vom 03.07.2003 (= Wagner 2003); Als Streiflicht in der SZ vom 11.06.2013 (= SZ 2013: 1); Als Witz in der FAZ am 10.08.2011 (= FAZ 2011b); Satire auf FAZ. NET am 28.01.2017 mit dem Titel „Nach dem Burnout kommt der Freakout“ (= Haupt 2017); als eine frühe kritische Reflexion auf den „,neuen Patiententyp“ in der FAZ (= möl. 1989); siehe auch SPIEGEL ONLINE (= Haas 2010; Meyehöfer 2001; SPIEGEL ONLINE 2012a); Oswald (2011, FAZ. NET), Martenstein (2012, ZEITmagazin 14/2012), alle Belege im QV unter 8.1.6.

347 Z. B. zwei Artikel in DER ZEIT Nr. 49/2011, im ersten wird `Burnout ‘ als ein „kulturelles Konstrukt“ bewertet und im zweiten Artikel als „akzeptierte Entschuldigung für Raubbau an den eigenen Kräften“ (= Albrecht 2011b und Pawelzik 2011); ähnlich SPIEGEL ONLINE Glaubitz (2011) und Knust (2012); im QV unter 8.1.6.

348 Siehe z. B. die Besprechung des Buchs „Burnout-Kids“ in der SZ am 11.07.2015 (= Bernd 2015: 50) oder die Fernsehkritik zur Sendung „Hart aber fair“ über das Thema „Burnout“ auf SPIEGEL Online am 15.11.2011 (= Patalong 2011); oder bezogen auf Buchrezensionen z. B. März (2010, DIE ZEIT) zum Buch von Miriam Meckel „Brief an mein Leben“ und Teutsch (2012, FAZ.NET) zu einem Buch von Nina Pauer oder die Besprechung des Romans „Acht Wochen verrückt“ von Eva Lohmann, der in der Rezension als „Burn-out-Roman“ betitelt wird (FAZ, 14.06.2011, = Hirsch 2011: 32) und die Besprechung des Films „Mit Burnout durch den Wald“ (= Mühl 2014, FAZ), des Films „Half Nelson“ (= UniSPIEGEL 2008) und einer Theaterlesung mit Burnout-Bezug (Schülke 2012, FAZ); oder mit Bezug auf eine Ausstellung, siehe Schulze (2012, SPIEGEL ONLINE) und Haas (2012, FAZ); oder mit Bezug auf Popmusik, die zum „Massenleiden ,Burnout““ passe, siehe Rapp (2012, SPIEGEL ONLINE), vgl. auch DER SPIEGEL (2011c) sowie Haas (2011, FAZ.NET) und Müller (2012, FAZ.NET); oder Kommentare zu Pressetexten über „Burnout“ (Malik 2002, unter manager-magazin/SPIEGEL); Steinkopf (2012) und Geyer (2012) in der FAZ/FAZ.NET, alle Belege im QV unter 8.1.6.

349 Z. B. auf FAZ.NET am 24.04.2014 „Belastungs-Test: Droht Ihnen der Burn-out“ (= Weiguny/ Nienhaus 2014c); „Burnout-Selbstprüfung: Riecht’s schon brenzlig?“ auf SPIEGEL ONLINE am 13.10.2013 (= Burisch 2013); im QV unter 8.1.6. 
Diese Liste der medialen Textsorten ist so unterschiedlich wie die Ziele, die diese Texte primär oder in Kombination verfolgen: über Neuigkeiten oder die individuellen, gesellschaftlichen und wirtschaftlichen Folgen des Phänomens informieren, den Gegenstand einordnen und bewerten oder Maßnahmen empfehlen.

Wolf-Andreas Liebert formuliert eine ähnliche Beobachtung in Bezug auf den Begriff des populärwissenschaftlichen Textes:

\begin{abstract}
Betrachtet man die Vielfalt der kommunikativen Ziele im Bereich der Vermittlung von Wissenschaft [...], so ist offensichtlich, dass die Texte so unterschiedlich sind, dass sie gar nicht ohne weiteres verglichen werden können. Damit ist aber zu fragen, ob man überhaupt von einer homogenen Textsorte „populärwissenschaftlicher Text“ sprechen kann. ${ }^{350}$
\end{abstract}

(Liebert 2002: 105)

Liebert schlägt daher vor schlicht von „Vermittlungstexten“ zu sprechen in der Lesart: „Text, mit dem Wissenschaft hinsichtlich eines bestimmten Ziels vermittelt wird“ (Liebert 2002: 106, im Original kursiv). Darüber hinaus gibt es Texte, in denen auf der Basis des aus der Wissenschaft stammenden Wissens Ratschläge vermittelt werden. Franke schlägt für diese Texte die Textsortenbezeichnung „ratgebender Aufklärungstext“ (Franke 1997: 375) vor, den er u. a. folgendermaßen beschreibt:

Mit der Veröffentlichung ratgebender Beiträge, so wird postuliert, unternehmen die Kommunikatoren in den Medien den Versuch, die breite Öffentlichkeit darüber aufzuklären, was unter bestimmten situativen Bedingungen zu tun möglich oder ratsam ist.

(Franke 1997: 374)

Auf die Kriterien zur (graduellen) Unterscheidung von Texten mit einer dominant ratgebenden Funktion gegenüber Texten mit einer dominant informierendaufklärenden Funktion, wird im folgenden Kapitel in Bezug auf Texte in populärwissenschaftlichen Zeitungen noch detailliert eingegangen.

In einigen der oben angeführten Textsorten, z. B. in Zeitungsmeldungen über prominente ,Burnout-Fälle` oder Krankschreibungsstatistiken, geht es zudem nicht (primär) darum, Wissenschaft zu vermitteln. Doch auch die Grenzen zwischen Ereignis- und Wissensvermittlung sind fließend, wenn beispielsweise in einer Meldung über die Häufigkeit der Zusatzdiagnose BuRNout auf Krankschreibungen das Wort Burnout hyperverlinkt ist und man dadurch auf eine Seite gelangt, die gleich einem Dossier alle Informationen zum Thema „Burnout“

350 In einer Fußnote verweist Liebert in diesem Zitat noch auf einen Aufsatz von sich (Liebert 1996) und auf eine Tabelle auf Seite 84 seiner Monografie. 
(Definition, Hintergrundberichte, etc.) gesammelt darstellt. ${ }^{351}$ Die unterschiedlichen Zielorientierungen der Texte verbinden sich mit den unterschiedlichen oben benannten Textsorten. Zusätzlich basieren diese Textsorten auf journalistischen und vermittlungssemantischen ${ }^{352}$ Praktiken und „Textsortenroutinen“ (Felder 2012: 120), die es zu berücksichtigen gilt, wenn in Kapitel 6 dieser Arbeit analysiert wird, welche Spuren (unwillkürlicher) sozio-kultureller sowie fachund berufsspezifischer Praktiken der Diskurs aufweist, die auf den Prozess des Definierens einwirken bzw. zusammen mit anderen Praktiken zu einer diskursiven Praxis des Definierens beitragen. An einem Beispiel sei illustriert, was damit gemeint ist:

Wenn einige Pressetexte im Korpus szenisch-narrativ anhand eines konkreten Fallbeispiels in das Thema „Burnout“ einführen, so kann man dahinter eine journalistische Texteröffnungspraktik der Textsorte Magazinbericht/Feature/Reportage oder eine aus Vermittlungs- und Identifizierungsgründen gewählte Personalisierungspraktik vermuten. In der weiteren Analyse soll daraufhin gezeigt werden, dass eine solche Texteröffnungspraktik auch definitorisches Potenzial birgt. Damit soll nicht unterstellt werden, dass der/die Textproduzent/-in bewusst das Ziel verfolgt, mit dieser Texteröffnung etwas zu definieren, sondern es geht um die Frage, inwiefern durch diese Texteröffnungspraktik beispielsweise typische narrative Ablaufmuster erzeugt werden, die den Gegenstand/Begriff >BURNOUT< umgrenzen und dadurch zu einer diskursiven Definitionspraxis von $>B U R N O U T<$ beitragen.

\subsubsection{Vermittlungstexte in populärwissenschaftlichen Zeitschriften und deren Onlinepräsenzen}

Im Unterschied zu den Nachrichten- und Vermittlungstexten in der tagesaktuellen Presse publizieren in populärwissenschaftlichen Zeitschriften ${ }^{353}$ zum einen auch verstärkt Expertinnen und Experten aus der psychosozialen Praxis ${ }^{354}$ zum Thema „Burnout“ und zum anderen ist die Zielgruppe der Rezipient/inn/en inhaltlich-thematisch stärker auf Personen, die an „Psychologie und [...] benach-

351 Vergleiche dazu im SPIEGEL folgende miteinander verlinkte Seiten: http://www.spiegel.de/gesundheit/diagnose/gesundheitsreport-dak-psychische-leiden-erreichen-hoechststand-a-885593. html und http://www.spiegel.de/thema/burn-out-syndrom/ (zuletzt eingesehen am 28.11.2019).

$352 \mathrm{Zu}$ Charakteristika von populärwissenschaftlichen/fachexternen Texten/Textsorten siehe ausführlich Becker (2001: 21ff.).

353 Zeitschriften sind „nicht tagesaktuell, dafür außerordentlich diversifizierter nach Themenbereichen und Adressatengruppen“ (Burger/Luginbühl 42014: 221).

354 Z. B. Beate Schulze, Soziologin und Psychologin, in „Psychologie Heute compact“, Heft 27 (2011) oder Peter Falkai, Professor für Psychiatrie und Psychotherapie, in „G\&G“, Dossier 1-2016: 20-22. 
barten Wissenschaften“"355 Interesse haben, eingegrenzt als in allgemeinen Tagesund Wochenzeitungen. Zeitschriften müssen zudem weniger aktuell sein und können spezielle Themen in einem weiteren Rahmen (z. B. einem Themenheft) ausführen. Ihr höherer Einzelheftpreis, die kleinere Auflage und ein im Vergleich

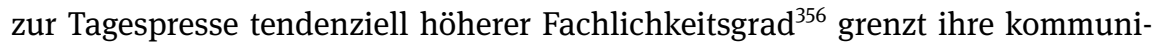
kative Reichweite ebenfalls weiter ein. Nach Liebert ist für unsere Wahrnehmung dieser Texte außerdem von Belang, dass diese Texte ,in einer explizit populärwissenschaftlichen Publikation gefunden werden“ (Liebert 2002: 105).

Bei der in dieser Arbeit ebenfalls untersuchten „Apotheken Umschau“ ist eine solche Einschränkung der kommunikativen Reichweite allerdings nicht erkennbar. Man kann zwar annehmen, dass die Zielgruppe an medizinischen und Gesundheitsthemen verstärktes Interesse zeigt, aber es kann kein genuines Interesse an psychologischen/psychiatrischen Themen abgeleitet werden. Des Weiteren besitzt die „Apotheken Umschau“ als kostenloses Kundenmagazin einen enorm hohen Verbreitungsgrad, was vermuten lässt, dass eine hohe kommunikative Reichweite angestrebt wird.

Ähnlich wie im vorherigen Kapitel gibt es nicht das eine feste Muster für Texte zum Thema „Burnout“ in den untersuchten populärwissenschaftlichen Magazinen. Es wurden aber folgende dominante Textsorten bzw. Textsortenmischungen ausfindig gemacht:

- $\quad$ Einige Texte arbeiten ebenfalls mit Praktiken des Magazinberichts bzw. Features, d. h. mit einem Wechsel von typischen personalisierten Fallschilderungen, Fotografien, Sacherläuterungen und Expertenstandpunkten ${ }^{357}$; In den Texten der „Apotheken Umschau“ zeigen sich die Merkmale von ratgebenden Texten am deutlichsten. Auf diese Merkmale wird im Anschluss an diese Aufzählung ausführlich eingegangen.

355 So z. B. die Selbstbeschreibung der Zeitschrift PH unter https://www.beltz.de/fachmedien/ psychologie/zeitschriften/psychologie_heute.html, zuletzt eingesehen am 28.11.2019.

356 In Texten von PH und G\&G finden sich Fachausdrücke wie systemischer Ansatz, Ressource, Attributionsstil, die nicht immer erläutert werden. Des Weiteren findet man besonders im Magazin G\&G Quellenangaben. Diese Zeitschriften richten sich von ihrem Selbstverständnis her neben interessierten Laien auch an Fachleute insbesondere aus der psychosozialen Praxis. Vgl. https://www.beltz.de/fachmedien/psychologie/zeitschriften/psychologie_heute.html und http://www.spektrumverlag.de/wp-content/uploads/2015/11/2016_GG-Mediadaten.pdf, zuletzt eingesehen am 28.11.2019.

357 Z. B. G\&G Dossier, Jan. 2016 (= Falkai 2016 und Keck/Holsboer 2016); PH 1/2016 (= Schönberger 2016: 18-21); PH 9/2011 (= Leggewie 2011); PH 4/2017 (= Kleinschmidt 2017); GEO WISSEN Nr. 48/2011 (= Frömel/Recht 2011: 43-57); G\&G 11/2005: 12-19 (= Kraft 2005); AU 12/1997-A (= AU 1997: 20-23); AU 06/2012-B (= AU 2012b: 19); AU 11/2011-B mit Fallschilderungen, Informationskästen und Expertenzitaten zur `Depression` (= Wolfrum 2011), alle Belege im QV unter 8.1.7. 
- Daneben gibt es Artikel, die das Thema „Burnout“ und den weitergefassten Themenkomplex rund um Arbeit/Beruf und Stress weniger an persönlichen Fallbeispielen, sondern im Sinne von Problem- oder Forschungs- und Studienberichten durch generalisierte Symptome und Ursachenkomplexe behandeln ${ }^{358}$ und/oder die Entstehung von >BURNOUT< aus verschiedenen psychologisch-medizinischen Forschungsrichtungen beleuchten (z. B. aus der biopsychologischen, (epi)genetischen, differentiellen oder arbeits- und organisationspsychologischen Perspektive) ${ }^{359}$. In den Texten der Magazine „Gehirn\&Geist“ (G\&G) und „Psychologie Heute“ (PH) erfolgt in dieser Textsorte durch den Bezug auf weitere psychologische Studien oder Expertenstimmen meist eine Verknüpfung zu anderen psychologischen/psychiatrischen Konzepten (z. B. `Depression`, `Typ-A-Verhalten`, >Attributionsstil`, >hardy personality`, >Nein-Sagen-Können`, >Perfektionismus` und >Idealismus etc.). ${ }^{360}$ Des Weiteren gibt es Texte, in denen >Burnout als Nebenthema erscheint zum Beispiel $\mathrm{zu}$ >chronischem Stress`, >Arbeitsbelastungく, >Konfliktbereitschaft` >steigenden Arbeitszeiten`, >Ungehorsam` oder >Entspannung « und >Achtsamkeit $\iota^{361}$

358 z. B. der erste Artikel über Burnout in der PH 10/1983, ein Vorabdruck aus dem Buch „Ausgebrannt“ (= Aronson/Pines/Kafry 1983), das aus dem Amerikanischen übersetzt wurde, in PH unter Aronson/Pines/Kafry (1983: 21-27); oder ein Beitrag von Matthias Burisch, der eine umfassende Übersicht zu Burnoutsymptomen aus der Monografie von Burisch ( $\left.{ }^{2} 1994 a\right)$ enthält, in PH 9/1994, (= Burisch 1994b: 22-26); oder ein Beitrag von Dagmar Ruhwandl zu Ursachen und Symptomen von Burnout in PH 5/2009 (= Ruhwandl 2009: 18-24); oder ein Beitrag von Beate Schulze, der beschreibt, wie man >Burnout erkennt und welche Risikokonstellationen es gibt, in PH 5/2009 (= Schulze 2009: 26-29); oder der Beitrag von Ute Eberle in GEO kompakt Nr. 40/2014 (= Eberle 2014: 20-29); alle im QV unter 8.1.7.

359 Vgl. z. B. G\&G Dossier 1/2016, das sowohl einen Artikel zu den biologischen Grundlagen von >BURNOUT< (= Reuter 2016: 43-47) als auch zu >BURNOUT< und Präventionsmaßnahmen aus arbeitsund organisationspsychologischer Sicht (= Ducki 2016: 80-85) und einen Artikel zum Einsatz neurobiologischer Erkenntnisse zur spezifischeren Behandlung von „Depression und Burnout“ (Keck/ Holsboer 2016) enthält. Nolte beschreibt ausführlich die Potsdamer Studie zur Lehrerbelastung von Uwe Schaarschmidt (= Nolte 2008, in PH 12/2008), alle Belege im QV unter 8.1.7.

360 Die Relevanz dieser Konzepte in Verbindung zur Burnout-Thematik wird beispielsweise in PH 5/2009 diskutiert (= Schulze 2009: 26-29); oder im Beitrag der AU (2011e) „Burnout: Perfektionisten sind gefährdet“; oder im Beitrag „Burnout ist eine Form der Depression“ in PH 12/2011 (= Tenzer 2011b: 30-33); oder in Bezug auf die sog. ,Selbstverbrenner“ und deren Idealismus und Perfektionismus im Beitrag von Ute Eberle in GEO kompakt Nr. 40/2014 (= Eberle 2014: 28); alle im QV unter 8.1.7.

361 Z. B. ein Beitrag zu steigenden Arbeitszeiten und psychischen Folgen in PH 11/2000 (= Kerber 2000: 30-35); oder zu hohen Arbeitsbelastungen in AU 10/2010-A (= AU 2010b: 7) und Folgen von Dauerstress (u. a. Burnout) in AU 12/2010-B (Essig 2010: 12); oder zu Entspannungstechniken (AU 05/2015-A: 22-26, = Aust 2015). 
- Interviews mit Experten/Expertinnen; ${ }^{362}$

- Berichte, Einschätzungen und Informationskästen zu Umfragen, Studien, Statistiken, Forschungsergebnissen und (falschen) Überzeugungen zum Thema „Burnout“ in der Bevölkerung; ${ }^{363}$

- (Problem-)Berichte über Burnout bei speziellen Berufs- oder Bevölkerungsgruppen wie z. B. bei Lehrer/innen, Therapeuten/Therapeutinnen, im sportlichen Bereich, bei Personen, die in der Kirche/Seelsorge arbeiten, oder bei Schulkindern/Jugendlichen; ${ }^{364}$

oder ein Beitrag zu „Ungehorsam - Prophylaxe gegen Burnout“ in PH 11/2011 (= Tenzer 2011a: 26-28); oder in der AU 5/2015 zur „Kunst des Entspannens“ (= Aust 2015: 22-26); alle im QV unter 8.1.7.

362 Z. B. ein Interview mit Christina Maslach in PH 10/1983: 24-25 (= Ernst 1983); ein Interview mit Matthias Burisch in der AU 10/2011-A (= Rotherbl 2011: 34-37); ein Interview mit Ruth Enzler Denzler in PH 5/2009, S. 30-31 (= Binkert 2009); ein Interview mit Hartmut Rosa in PH 1/2013 (= Schönberger 2013); oder das Interview mit Gunther Schmidt in PH 1/2016 (= Schönberger 2016b: 22-24); siehe auch AU 2014; alle im QV unter 8.1.7.

363 Z. B. fasst der Autor Christian Wolf in PH 10/2013 Ergebnisse einer Umfrage von Johannes Hamann et al. zusammen, in der das Verständnis des Begriffs `Burnout` in Psychiatrie und Psychotherapie erfragt wurde (= Wolf 2013: 11, im QV unter 8.1.7). Die Studie, auf die sich der Artikel in PH bezieht, ist ebenfalls im fachlichen Textkorpus dieser Arbeit enthalten, und zwar im Bereich der Zeitschrift „Der Nervenarzt“ unter Hamann/Parchmann/Mendel (2013: 338-843, im QV unter 8.1.4); siehe auch den mit „Faktencheck“ übertitelten Beitrag „Elf Mythen über Burnout“ in G\&G 2016 (= Melchers/Plieger 2016: 13-18) oder der Artikel „Hier irrt der Psychosomatiker“ in PH 08/2014, in dem der ,Mythos“ „hart arbeiten“ führe zu Burnout berichtigt wird, indem dies nur auf negativen übermäßigen Stress bezogen wird (= Paulus 2014: 79); eine Nachricht zu Umfrageergebnissen und Studien findet man zum Beispiel in AU (2011c) „Burnout: Jeder zehnte deutsche Berufstätige gefährdet“ auf www.apotheken-umschau.de vom 10.10.2011 (Stand: 24.11.2012) sowie AU 2010a und AU 2015; oder Heiko Ernst schreibt im Editorial der PH 4/2013 über den „Stressreport Deutschland 2012“ (= Ernst 2013a: 3); siehe im QV unter 8.1.7.

364 Z. B. ein Beitrag von M. Burisch, Matthias „Der leere Lehrer“ in der Hamburger Lehrerzeitung 1/1985 (= Burisch 1985: 8-11); oder „Nicht jede Lehrkraft ist gestreßt“ in PH 12/1997 (= Mrechar 1997: 42); oder „Ausgebrannt im Klassenzimmer“ in PH 4/2000 (= Smolka 2000: 38-43), siehe auch PH 12/2008 (= Nolte 2008); oder der Beitrag „Ein Lehrer kann seine Schüler nicht einfach entlassen, wenn sie ihm nicht passen“ mit dem Interviewpartner Joachim Bauer in $\mathrm{PH}$ 1/2004 (= Krumpholz-Reichel: 34-38); oder der Beitrag „Berufsrisiko Psychotherapie: Ist Leid ansteckend?“ in PH 6/2005 (= Speck/Horsch 2005: 64-69); oder ein Beitrag zu Burnout in Pfarreien in PH 10/2012 (= Ustorf 2012: 34-37); oder zu „Schulstress bei Jugendlichen“ auf: www. apotheken-umschau.de vom 23.11.2012 (= Schuster 2012); „Stress im Kinderzimmer“ PH 2/2003 (= Sentker 2003: 46-51) oder der Beitrag „Strategie, Taktik, Burnout - Zur Psychologie des Fußballtrainers“ in PH 6/2014 (= Metzger 2014: 70-74); oder der Beitrag zu Burnout/Depression im Hochleistungssport in PH 9/2011 (= Ustorf 2011: 64-69); oder der Beitrag „Unsere erschöpften Kinder“, ein Interview mit Michael Schulte-Markwort in PH 9/2016 (Otto 2016; 12-15); alle im QV unter 8.1.7. 
- Vorworte in Zeitschriftenausgaben, in denen „Burnout“ Schwerpunktthema ist $^{365}$

- Fragebogen. $^{366}$

Die Magazine „Psychologie Heute“ sowie „Gehirn\&Geist“ unterscheiden sich von der „Apotheken Umschau“ und anderen massenmedialen Beiträgen durch die Komplexität der Behandlung des Gegenstands `BuRnout‘ und durch die Verwendung von Fachausdrücken, die nicht immer erläutert werden. Da die Beiträge in den Magazinen überwiegend mindestens zwei Magazinseiten füllen, sind sie von ihrem Umfang her am besten mit Titelgeschichten aus dem SPIEGEL oder ausführlichen Berichten in Zeitungen z. B. aus dem Ressort „Wissen“/,Natur und Wissenschaft“ oder „Beruf und Chance“/,Job und Karriere“ vergleichbar. ${ }^{367}$ Die Texte in populärwissenschaftlichen Magazinen enthalten häufiger detaillierte Symptomlisten, ausführliche Beschreibungen von Burnout-Stadien, Erläuterungen zu verschiedenen ätiologischen Theorien und (fachliche) Ratschläge zur Prävention und Therapie.

Daher könnte man viele dieser Texte mit Lüger (21995: 71f.) auch als „instruierend-anweisend“ beschreiben oder der von W. Franke beschriebenen Textsorte „ratgebende[r] Aufklärungstext“ zuordnen, die Wilhelm Franke folgendermaßen charakterisiert:

Mit der Veröffentlichung ratgebender Beiträge, so wird postuliert, unternehmen die Kommunikatoren in den Medien den Versuch, die breite Öffentlichkeit darüber aufzuklären, was unter bestimmten situativen Bedingungen zu tun möglich oder ratsam ist. Als primäres

365 Z. B. die Vorworte von Heiko Ernst „Ach, wir sind des Treibens müde“ in PH 10/2002 (= Ernst 2002b: 3); oder „Ich möchte lieber nicht ... “ in PH 11/2011 (Ernst 2011: 3); siehe auch Ernst (2002a; 2010; 2013a/b; 2014a/b) oder die Vorworte von Ursula Nuber in PH 1/2016, die mit Sätzen beginnen wie: „Wir sind die Ausgebrannten. Unser Selbst ist erschöpft.“ (Nuber 2016a: 3) siehe auch Nuber (2015 a/b/c; 2016b).

366 Auf der Onlinepräsenz der AU (siehe AU 2017c/d und AU 2012-A: 6-7); „Sind Sie ausgebrannt?“ in G\&G (2016: 23); Nelting/PH, 01/2016: 25, ein Nachdruck aus Nelting (52014); Test zur Messung der Stressbelastung bzw. Burnoutgefährdung in GEOWISSEN 48/2011: 124-125; alle im QV unter 8.1.7.

367 Z. B. der Beitrag „Das zehrt an den Nerven ,Burnout’: Hat die Diagnose Methode, oder ist es Mode?“ in der FAZ vom 6.10.2010: S. N1 (= Kaulen 2010); oder der Beitrag „Völlig ausgebrannt im Klassenzimmer“ in der FAZ vom 21.03.2007: S. N2 (= Lutterotti 2007), jeweils in der Rubrik „Natur und Wissenschaft“; oder ein Artikel in der SZ vom 22.10.2011 mit dem Titel „Die Burn-out-Hysterie“ zur Einordnung von Burnout neben anderen psychiatrischen Diagnosen aus dem Ressort „Wissen“ (= Weber 2011: 24); Beispiele aus dem Ressort: Beruf/Job und Chance/Karriere: FAZ, 19.02.2011: S. C1 „Ausgebrannt am Scharmützelsee“ (= Loll 2011); oder „Müde, antriebslos, zynisch“ in der SZ vom 30.10.2012 (= Haas/Wolff 2012); alle im QV unter 8.1.6. 
Ziel von Aufklärung wurde dabei die Herbeiführung einer Änderung im kognitiven System der Adressaten bestimmt. Durch den Transfer von Wissen in den Spielarten des ,FrageAntwort-Spiels‘ bzw. der ,Belehrung، sollen auf seiten der Rezipienten die Bedingungen der Möglichkeit geschaffen werden, daß sich jene in entsprechenden Handlungssituationen vernunftgerecht zu entscheiden und autonom zu handeln vermögen.

(Franke 1997: 374, Kursivierung im Orig.)

Für Franke unterscheiden sich ratgebende Aufklärungstexte „nicht in ihren Darbietungsformen von massenmedialen Beiträgen anderen Typs, sondern hinsichtlich dessen, was in ihnen vermittelt“ wird: und zwar nicht (nur) „theoretisches Wissen“"368, sondern auch Bestände ,an ,nicht-verbindlichem Handlungswissen““ (Franke 1996: 259). Nicht-verbindlich sei dieses Wissen, weil nicht vermittelt werde, ,welches Handeln in einer bestimmten Situation erlaubt, verboten oder vorgeschrieben ist“ (Franke 1997: 157), sondern „was im Hinblick auf die Erlangung eines bestimmten Zielzustands erfahrungsgemäß zu tun bzw. zu unterlassen sinnvoll, ratsam oder zweckmäßig ist“ (Franke 1997: 159). Franke ordnet die ratgebenden Aufklärungstexte der „Klasse der assertiven, informationsübermittelnden Textsorten“ zu (Franke 1997: 161). Er wendet sich in seiner Arbeit z. B. gegen Klassifizierungen von Hindelang (1978), Brinker (1985) oder Rolf (1993), die diese Texte den (bedingt) direktiven Textsorten zuordnen. ${ }^{369}$ Franke begründet seine Klassifizierung damit, dass

entscheidende konstitutive Bedingungen für Aufforderungshandlungen [...] nicht bzw. nicht notwendigerweise gegeben sind, etwa: daß der Kommunikator will, daß der Adressat eine bestimmte Handlung ausführt und daß der Vollzug der Sprechhandlung als Versuch des Kommunikators gilt, Adressaten dazu zu bewegen, die im propositionalen Gehalt spezifizierte Handlung auszuführen. ${ }^{370}$

(Franke 1997: 164)

368 Franke versteht unter theoretischem Wissen „Wissen über Sachverhalte“ bzw. „Faktenwissen“, das dem „Handlungswissen“ bzw. „prozeduralem Wissen“ gegenübergestellt wird. Vor den Ausführungen zum Verhältnis von Wissen und Praxis in dieser Arbeit wird allerdings bezweifelt, dass man diese Wissensarten so strikt voneinander in der Vermittlungssituation trennen kann. Vgl. Kap. 2.1.1, 2.2.1 und 4.1.1 dieser Arbeit.

369 Götz Hindelang (1987: 378) spricht in Bezug auf „Anleitungen“ von „NICHTBINDENDEN AUFFORDERUNGEN“ (Großschreibung im Orig.) und zu „Ratschlägen“ schreibt Hindelang, dass sie „und ihre sprachlichen Ausdrucksformen innerhalb des Systems der Aufforderungshandlungen beschrieben werden können (ebd.: 447); Eckard Rolf (1993: 257f.) beschreibt Textsorten wie „Rat“ und „Ratgeber“ im Kapitel „Nicht-bindende Textsorten bei Rezipienteninteresse“ und charakterisiert diese als „,[N]icht bindende direktive Textsorten“; Brinker (1985: 103f.) ordnet instruierende Texte einer direktiven „Wenn-dann-Relation“ zu (vgl. auch Brinker/Cölfen/Pappert ${ }^{8}$ 2014: 111). 370 Fußnote im Original verweist auf weitere Ausführungen zum Aufklärungsbegriff in Kap. 2, Abschnitt 2.1 von Franke 1997. 
Das primäre Ziel des Emittenten der Ratgebung in der unidirektionalen Kommunikationssituation der Massenmedien sei es, ein beim Adressaten vorhandenes oder unterstelltes Wissensdefizit diesem bewusst $\mathrm{zu}$ machen und $\mathrm{zu}$ beseitigen, d. h. ,aufzuklären‘. Es gehe dabei darum, ,durch die unidirektionale Weitergabe von Wissen auf seiten des Rezipienten die Bedingungen der Möglichkeit dafür zu schaffen, daß sich jener bewußt und aus eigener Einsicht in entsprechenden Situationen für oder gegen den Vollzug einer bestimmten Handlung entscheidet“ (Franke 1997: 164). Dazu würde darüber hinaus gehören, die „Perspektivität von Wissensbeständen offen[zu]legen“ (Felder 2013: 15ff.).

Auch wenn die Ausführungen von Franke insofern überzeugend sind, dass die Autoren/Autorinnen von Ratgebertexten unidirektional eine prinzipiell anonyme, Leserschaft adressieren und dadurch auch nicht den „effektiven Rezipienten“ auffordern können, ihren Ratgebungen Folge zu leisten, so adressieren sie dennoch eine „intendierte“ Leserschaft, ${ }^{371}$ und es wirkt kontraintuitiv, folgende Textbestandteile aus der „Apotheken Umschau“ als Teile einer rein informierend-darstellenden Textfunktion zu interpretieren:

Die betriebsbedingten Risikofaktoren lassen sich meist kaum verändern. Anders die persönlichen: Hier können Sie einiges tun, um die Erschöpfungsspirale anzuhalten.

- Überprüfen Sie Ihre Erwartungen an sich selbst, und akzeptieren Sie, dass auch Sie nicht perfekt sein können.

- Setzen Sie sich überschaubare, realistische Ziele [...].

(Apotheken Umschau 2005b: 40)

Diese direkte Ansprache der Rezipientinnen und Rezipienten im Modus des Imperativs kann als Indiz dafür verstanden werden, dass der Autor/die Autorin bei der Person, die sich angesprochen fühlen soll, eine spezifische Verhaltens- und Handlungsänderung erzielen möchte oder diese zumindest als geeignete Maßnahme empfiehlt. Die Person wird aufgefordert, die eigenen Erwartungen „zu überprüfen“ und in Bezug auf das Thema das eigene Handeln zu reflektieren. Dadurch wird nicht nur informiert oder aufgeklärt, sondern der Emittent fordert den Adres-

371 Burger und Luginbühl ( $\left.{ }^{4} 2014\right)$ unterscheiden zwischen dem „effektiven“ und dem „intendierten“ Rezipienten: „Jeder, der eine Zeitung liest, eine Sendung hört oder anschaut, ist ein ,effektiver' Rezipient, gleichgültig ob er das Medienprodukt nur zufällig oder ganz bewusst und absichtlich wahrnimmt. Demgegenüber kann man als ,intendierte Rezipienten“ jene Rezipientengruppe bezeichnen, die vom Kommunikator explizit oder implizit anvisiert wird. (Ob diese Rezipienten erreicht werden, ob der intendierte zum effektiven Rezipienten wird, hängt dann von vielen, zum größten Teil außerlinguistischen Faktoren ab.) Uns scheint es richtig, mit dem in der Linguistik gängigen Terminus ,Adressat' den vom Sender intendierten Empfänger zu bezeichnen, und nicht primär den ,effektiven` Rezipienten“ (Burger/ Luginbühl ${ }^{4} 2014:$ 11). 
saten auf, die vorgeschlagenen Ratschläge mit dem eigenen Leben abzugleichen und im Fall einer Übereinstimmung diese Ratschläge weiter umzusetzen. ${ }^{372}$

Unabhängig davon, ob man den deontischen Gehalt von dieser Art von Texten stärker im illokutionären Gefüge oder - wie Franke - in den spezifischen Strukturen des vermittelten Handlungswissens und dessen Modalitäten wiederfindet, wird in dieser Arbeit im Folgenden von Ratgebertexten gesprochen, wenn in diesen Texten expliziert wird, welche Handlungen, Verhaltensänderungen oder Handlungsabfolgen ausgeführt werden müssen/können/soll(t)en, wenn in einer bestimmten Situation ein spezifischer Zustand (wieder) herbeigeführt, beseitigt, bewahrt oder verhindert werden soll.

Tendenziell indizieren $u$. a. folgende sprachliche Merkmale im vorliegenden Korpus dominant ratgebende Texte:

- Sprachliche Mittel zur Anzeige deontischer und optionaler Modalität: „Überprüfen Sie Ihre Erwartungen [...].“; „Am Ende einer Bestandsaufnahme kann durchaus die Erkenntnis stehen, daß der aktuelle Beruf [...] gewechselt werden muss“ (AU 05/2005-B: 40 (= AU 2005b); AU 10/1995-B: 12 (= AU 1995d; Unterstreichungen T.S., beide im QV unter 8.1.7). „Erfolgsversprechende Bewältigungsstrategien erfordern [...] eine gründliche und vor allem ehrliche Bestandsaufnahme“ (AU 2005b: 40); „Anti-Stress-Strategie Nr. 1: Lernen Sie den Feind kennen“ (PH 7/2000 = Ernst 2000: 24, Unterstreichung T.S. im QV unter 8.1.7);

- Sprachliche Mittel, die den Leser/die Leserin in das Geschehen des Textes einbeziehen und gleichzeitig allgemeine Gültigkeit indizieren: AgensAusdrücke, die zwischen Verallgemeinerung und Singularität und generischen und individuellen Bedeutungskomponenten changieren: z. B. wenn die Geschichte eines Individuums, dessen Eigenname genannt wird oder das mit ich spricht, exemplarischen Charakter erhält (z. B. AU 2012b; AU 2017a; Falkai 2016 in G\&G); wenn der Adressat zwar direkt angesprochen wird „Hier können Sie einiges tun, um die Erschöpfungsspirale anzuhalten“, aber das Pronomen Sie durch das verallgemeinernde Pronomen man substituierbar ist (vgl. Franke 1997: 227) oder Phasen mit den Pronomen uns und wir „Was uns fehlt, ist regelmäßige Entspannung“ oder „Durch geeignetes Stressmanagement können wir [...] unser Gefühl für die eigene Wirksamkeit erhöhen“ (PH 2/2002 = Nuber 2002: 24), wodurch eine Gruppe aus Autor/Autorin und Lesenden konstituiert wird.

372 Auch Greta Stanaitytė weist darauf hin, dass Medientexte, die Themen wie Gesundheit, Ernährung oder Hobbys behandeln, häufig „neben dem darstellenden funktionalen Anteil auch einen evaluativen oder appellierenden annehmen“ (Stanaitytė 2005: 46). 
- Tendenziell Elemente eines informelleren Stils: „[S]ie werden in die ,Normalität‘ zurückkatapultiert“ (Freudenberger/North ${ }^{9} 2002$ : 13, im QV unter 8.1.5); „wie ein Hamster im Laufrad“ (PH compact 27/2011: 41);

- Motivierungssignale: „Solange man diese Anzeichen wahrnimmt [...], ist die Burn-out-Gefahr meist schnell gebannt“ (PH 5/2009, = Schulze 2009: 26). ${ }^{373}$

Ratgebertexte richten sich einerseits unidirektional an eine anonyme Leserschaft, andererseits adressieren sie eine Gruppe von Lesern/Leserinnen, die sich von dieser Wissensvermittlung angesprochen fühlen (sollen), und damit adressieren sie gewissermaßen auch die einzelne (anonyme, aber typisierte) Leserperson, denn nur diese kann das vermittelte Handlungswissen umsetzen. Diese Mehrfachadressierung findet sich zwar auch in anderen Vermittlungstexten, die weniger auf die Weitergabe von praktischem Handlungswissen fokussieren, aber Ratgebertexte konstruieren gewissermaßen ein individuelles Handlungssubjekt als verantwortliche Person und beziehen den Leser/die Leserin in diese Konstruktion mit ein.

In diesem Zusammenhang ist das folgende syntaktische Muster interessant:

Wer an seiner Arbeit keinen Spaß mehr findet, sich immer schlechter konzentrieren kann, sich ständig müde und erschöpft fühlt, der braucht vielleicht Hilfe von außen.

(AU 12/1997-A: 20, im QV unter 8.1.7)

Dieses an die Struktur von Sprichwörtern erinnernde syntaktische Muster, das in Texten der Apotheken Umschau ${ }^{374}$, aber auch in Texten mit dominant ratgebender Funktion der Psychologie Heute oder FAZ erscheint, ${ }^{375}$ bietet dem Leser/der Leserin eine einerseits singuläre, aber zugleich generische Identifizierungs-Schablone, ob er/sie sich zu dieser Gruppe ,Betroffener` zu zählen hat oder nicht.

373 Alle Belege der PH und AU sind im QV einsehbar unter 8.1.7.

374 Vgl. z. B. auch das folgende Beispiel aus AU (2011d): „Gesund leben: Wer auf eine ausgewogene Ernährung und feste Schlafenszeiten achtet, und sich regelmäßig bewegt, stärkt den Körper und das eigene Wohlbefinden“; oder „Wer sich bewegt und den Geist offen hält, ist weniger anfällig für Stress und Krankheiten“ (AU 9/2001: 17); im QV unter 8.1.7.

375 Dabei wird das Muster entweder mit wer ... , der realisiert oder das der wird ausgelassen: In einem Artikel der FAZ vom 26.05.2010 mit dem Titel „Wie lässt sich Burnout verhindern?“ findet sich zum Beispiel die Aussage: „Wer sich nur über seine Arbeit definiert, kann es nur schwer akzeptieren, wenn er oder sie auf einmal nicht mehr ,funktioniert‘ und körperlich und seelisch an seine Grenzen gekommen ist“ (= Stock 2010: B4, im QV unter 8.1.6, FAZ); In PH findet man die folgenden Beispiele: ,Wer gelassener leben will, sollte lernen, die ,inneren Antreiber ' in Schach zu halten“ (PH, 2/2002 = Nuber 2002: 20); „Wer im Beruf einem hohen Burnoutrisiko ausgesetzt ist, sollte gelegentlich den Mut zu gut dosiertem Ungehorsam aufbringen“ (PH 11/2011 = Tenzer 2011a: 26) (Unterstreichungen T.S.; im QV unter 8.1.7, PH). 
Diese Art der sprachlichen Realisierung des Problems Burnout indiziert die Lesart, dass die Leser/innen, die angesprochen werden sollen, sich mit dem beschriebenen Problemzustand identifizieren bzw. ihr Verhalten und ihre Emotionen mit den beschriebenen Zuständen abgleichen sollen. Im Fall von Texten über >BuRNOUT` beinhaltet dies auch, dass die Leser/innen eigene problematische und bis dahin vielleicht nicht reflektierte Verhaltensweisen, Eigenschaften, Rollenbilder etc. erkennen und auf ihre Situation übertragen müssen. Diese Hürde für den Einzelnen wird wiederum abgeschwächt, wenn dieser mit dem Problem nicht allein ist, wie folgender Texteinstieg vermittelt:

\section{„Burnout: Was uns gefährdet. \\ Was uns schützt}

Wie erkennt man, ob eine Erschöpfung ganz normal ist oder ob man bereits am Burnoutsyndrom erkrankt ist? Und liegt es nur an der vielen Arbeit und den hohen Alltagsanforderungen, wenn man ausbrennt?

Das Gefühl kennen viele: Man ist müde schon beim Gedanken an die Arbeit, [...], man hat den Eindruck, man drehe sich im Kreis wie ein Hamster im Laufrad, [...]. Ist das bereits ein Burnout? ～(Psychologie Heute compact 27/2011 = Schulze 2011: 41, im QV unter 8.1.7)

Durch das inklusive uns wird markiert, dass „Burnout“ im Prinzip alle Menschen betreffen kann, und der Leser/die Leserin wird als Teil dieser Gruppe angesprochen. In ähnlicher Weise fungiert hier das Pronomen man, das für eine Einzelperson, sofern sie in dieser Situation „stellvertretend für jedermann genommen werden kann“376, steht. Die Fragen zu Beginn des Textes, die in dieser direkten Form in einem Interview an einen Experten/eine Expertin gestellt werden könnten, fingieren einen direkten Beratungsdialog.

Diese Varianz von sprachlichen Mitteln zur Erzeugung von Identifizierungsfolien und persönlicher Nähe zeigt sich im fachexternen Korpus insbesondere in Ratgebertexten, in denen nicht-verbindliches Handlungswissen für den ,Einzelnen' als Vertreter einer burnoutgefährdeten Person transportiert wird. Dieser anvisierte Rezipient wird dadurch als Typus ,definiert‘. Die Grenze zwischen stärker informierenden gegenüber stärker ratgebenden Vermittlungstexten ist fließend. Tendenziell zeigen sich in den Texten der „Apotheken Umschau“ und Ratgeberbüchern (z. B. Freudenberger/Richelson 1980a/1980b; Nelting 2010) am meisten der besprochenen charakteristischen Sprachgebrauchsformen.

376 Siehe die Bedeutungsangabe zum Indefinitpronomen man auf Duden online unter: http:// www.duden.de/rechtschreibung/man_jemand_irgendeiner_irgendeine (zuletzt eingesehen am 1.12.2019). 


\subsubsection{Onlineplattformen: Wikipedia, NetDoktor, Onmeda}

Nachdem in den letzten Unterkapiteln ausführlich verschiedene Erscheinungsformen von fachinternen und -externen Medien zu >BURNOUT< beschrieben wurden, die gedruckt oder zumindest meist auch gedruckt vorliegen, widmet sich dieses Unterkapitel Texten, die auf öffentlich zugänglichen Internetplattformen veröffentlicht wurden: Textfassungen zum Gegenstand/Begriff `BURNOUT der OnlineEnzyklopädie Wikipedia und Texte der Gesundheitsinformationsplattformen „NetDoktor“ und „Onmeda“ (siehe im QV unter 8.1.8). Diese Texte wurden in das Untersuchungskorpus aufgenommen, da eine „Kantar-Emnid-Befragung“ aus dem Jahr 2017 im Auftrag der Bertelsmann-Stiftung ergeben hat, dass sich Patientinnen und Patienten am häufigsten „Gesundheitsinfos auf Wikipedia und anderen Online-Lexika“ suchen. Die Bertelsmann-Studie „Gesundheitsinfos“ (Haschke/ Westrick/Schwenk 2018), in der u.a. Daten aus 36 Tiefeninterviews gewonnen wurden, hat zudem ergeben, „dass kommerzielle Angebote wie apothekenumschau.de (Wort und Bild Verlag) und netdoktor.de (Verlagsgruppe Georg von Holtzbrinck) einen hohen Bekanntheitsgrad“ haben und von den Befragten ,überwiegend gute Noten, auch wegen der Art der Ansprache“ erhalten (ebd.: 7). In das Korpus wurden daher neben Artikeln aus der gedruckten Zeitschrift „Apotheken Umschau“ auch Artikel der Onlinepräsenz dieser Zeitschrift mit aufgenommen (siehe Kap. 5.1.3 und 5.3.3.4).

Von der Online-Plattform Wikipedia wurden seit dem ersten Eintrag zum Stichwort Burnout am 12. März 2004 bis 2019 in Jahresabschnitte sechzehn Versionen des Eintrags zum Stichwort Burnout in das Untersuchungskorpus aufgenommen, um die Entwicklung der Wikipediaeinträge nachvollziehen zu können. Beispielsweise ist für die spätere Analyse einer Praxis des Definierens im BurnoutDiskurs von Interesse, wie der definierende Abschnitt über die Jahre hinweg verändert wird und aus welchen Quellen sich diese Definitionsabschnitte speisen. Die verschiedenen Textversionen zum Stichwort Burnout in der Wikipedia sind kollaborativ verfasst und orientieren sich an Schreibpraktiken beispielsweise zu Aufbau und Stil, die in den Statuten der Wikipedia empfohlenen werden. ${ }^{377}$ Die aktuelle Version des Wikipedia-Artikels zu $>$ Burnout umfasst 1.) einen Vorspann, der in den Richtlinien der Wikipedia „Definition und Einleitung“ genannt wird (ebd.) 2.) ein Inhaltsverzeichnis, 3.) den Haupttext mit zehn Unterkapiteln (u. a. zur „Geschichte“, zu „Symptomen“, „Phasen des Burnout-Syndroms“ etc.), 4.) Verweise auf weitere massenmediale Informationsangebote, 5.) Hinweise auf

377 Vgl. die Informationen der Wikipedia unter dem Titel „Wie schreibe ich gute Artikel“: https://de.wikipedia.org/wiki/Wikipedia:Wie_schreibe_ich_gute_Artikel, (zuletzt eingesehen am 17.4.2017). 
sachverwandte Artikel in der Wikipedia-Enzyklopädie, 6.) eine Liste ausgewählter Fachliteratur und 7.) einen Fußnotenapparat. In der Fußleiste steht des Weiteren ein Hinweis, dass dieser Artikel „nicht der Selbstdiagnose“ diene und „,keine Arztdiagnose“ ersetze, eine Katalogisierungsnummer und Anzeige, dass der Artikel über Burn-out in der Wikipedia in die „Kategorie: Psychische Störung“ fällt. Die Binnengliederung im Hauptteil weist Ähnlichkeit zu Überblickstexten in Fachund populärwissenschaftlichen Zeitschriften und Handbüchern/Lehrbüchern im Untersuchungskorpus auf (Unterkapitel zur Symptomatik, Diagnose bzw. Messung, Prävention etc.). Vergleicht man den Wikipedia-Artikel jedoch darüber hinaus mit gedruckten Artikeln in Lehr- oder Handbüchern oder Enzyklopädien sowie Fachlexika, so lassen sich hinsichtlich Produzent/inn/en, Adressat/inn/ en und medialen sowie inhaltlich-formalen Aspekten folgende charakteristische Aspekte ausfindig machen:

- Der Wikipedia-Eintrag zu ১Burnout ist umfangreicher im Vergleich zu vielen der untersuchten Einträge in Fachlexika und Handbüchern oder Enzyklopädien. In letzteren ist dem Thema „Burnout“ teilweise nur ein Abschnitt innerhalb eines anderen Überthemas gewidmet.

- Der Wikipedia-Artikel unterscheidet sich bezogen auf seine hypertextuelle Anordnung in der „Anzahl und Art der Verweise“ (Schmitz 2015: 103) von gedruckten Texten in Fachlexika und Handbüchern. Denn - anders als in letzteren - werden nicht nur andere Fachtermini, sondern auch Ausdrücke wie englisch, Erfolg, Häufigkeit wikipediaintern verlinkt. ${ }^{378}$ Im Abschnitt „Einzelnachweise“ wird des Weiteren nicht nur auf Fach- bzw. Ratgeberliteratur, sondern auch auf Internetseiten von Universitäten, Landesämtern oder Bundesanstalten und damit auf gesellschaftspolitische Diskursakteure verwiesen. Diese Internetseiten sind ,nur einen Klick entfernt ‘ und laden dadurch stärker zum „Herumspringen“ (Adamzik 2002: 178) ein als die Verweise in gedruckten Werken.

- Die gedruckten Texte sind in einem stark verdichteten, kontinuierlichen Duktus „aus einem Guss“379 meist von im Fach anerkannten Spezialisten geschrieben. Der Wikipediaeintrag hingegen wird von vielen Personen meist unter Pseudonym bearbeitet ${ }^{380}$, und der Fließtext erweckt stärker den Eintrag

378 Im Wikipedia-Artikel zu „Burn-out“ werden beispielsweise auch Wörter/Mehrworteinheiten wie „englisch“ oder „University of California“ verlinkt.

379 Vgl. die Beobachtung von Schmitz, der den Eintrag zum Stichwort „Sprachwissenschaft“ der gedruckten Brockhaus-Enzyklopädie (1998) mit dem Wikipedia-Artikel „Sprachwissenschaft“" vergleicht (Schmitz 2015: 103f.)

380 Dadurch sind die fachlichen und beruflichen Hintergründe bei vielen schreibenden oder bearbeitenden Personen nicht nachvollziehbar. Im Jahr 2004, in dem der Artikel angelegt und 
der Montage von mehr oder weniger deutlich gekennzeichneten Zitaten und Aufzählungen als gedruckte Lexikoneinträge (siehe dazu Kap. 6.2.2.1 und 6.2.3.2). Schmitz vermutet für Texte der Wikipedia insgesamt, dass „die VerfasserInnen [...] nicht“ erwarten, „dass der gesamte Artikel vollständig gelesen wird“ (Schmitz 2015: 105). Die zielgerichtete Rezeption wird ferner durch das Inhaltsverzeichnis, Zwischenüberschriften und typografische Mittel unterstützt (vgl. ebd.). Das Autorenkollektiv kommuniziert an eine Vielzahl von Rezipientinnen und Rezipienten. Zwischen „den Individuen, die das Publikum bilden“, müssen wie bei Pressetexten „keine Gemeinsamkeit bestehen“ (Burger ${ }^{4} 2014 /$ Luginbühl: 8). ${ }^{381}$

- Der Wikipediaeintrag weist eine zu den Fachlexika und Handbuchartikeln vergleichbare Dichte an psychologisch-medizinischen Fachtermini auf (z. B. Depersonalisierung, Selbstwirksamkeitserwartung). Diese werden in der Regel verlinkt, dennoch verringert dies die inhaltliche Reichweite, da der Text ohne Hin- und Herspringen eher dem fachsemantischen Pol zuzuordnen ist.

Als Ziel ihrer Artikel formuliert die Wikipedia-Enzyklopädie: „Ziel des Enzyklopädieprojektes ist die Zusammenstellung bekannten Wissens. “382 Die Grundfunktion des Wikipedia-Eintrags zu „Burn-out“ ist konform zu diesem Anspruch informierend-aufklärend. In der Feinanalyse der Wikipedia-Texte in den Kapiteln 6.2.3.2 und 6.2.4.2 wird ein besonderes Augenmerk auf Praktiken der Quellenauswahl, intertextuellen Bezugnahme und Zusammenstellung gelegt werden, weil diese, wie zu zeigen sein wird, über die Wikipedia-Plattform in besonderem Maße verknappende und unifizierende Wirkung entfalten können.

erweitert wurde, kann bei keinem Benutzer der berufliche Hintergrund eingesehen werden, da einige nur über IP-Adressen ihre Bearbeitungen einstellen oder in ihrem Profil diese Information nicht angeben. Einige Benutzer/innen in der späteren Artikel-Versionengeschichte zu „Burn-out“ veröffentlichen jedoch ihren beruflichen Hintergrund, z. B. Benutzer H.-P.Haack gibt folgende Informationen an: „Hans-Peter Haack (*1940), Neurologe und Psychiater in Leipzig und Heidelberg bis 2005 https://de.wikipedia.org/wiki/Benutzer:H.-P.Haack (Profil-Stand: 22.4.2017).

381 In welchem Ausmaß Wikipedia beispielsweise auch von Wissenschaftlern/Wissenschaftlerinnen und in der Praxis tätigen Fachleuten genutzt wird, ist bisher zu wenig erforscht. Eine Umfrage im Auftrag von „Goportis“ (= Leibniz-Bibliotheksverbund Forschungsinformation) unter Wissenschaftlerinnen und Wissenschaftlern der „Fachbereiche Agrar-, Forst-, Ernährungswissenschaften, Humanmedizin/Gesundheitswissenschaften, Tiermedizin/Veterinärmedizin, Umweltschutz, Biomedizin und Pharmazie“ aus dem Jahr 2015 hat ergeben, dass Wikipedia auch im wissenschaftlichen Bereich zum Beispiel zum Einstieg in ein Thema oder zur Sichtung von Quellenverweisen genutzt wird (Dzeyk 2015: 8; 33ff.).

382 https://de.wikipedia.org/wiki/Wikipedia:Was_Wikipedia_nicht_ist, (zuletzt eingesehen am 3.12.2019). 
Neben Einträgen der Wikipedia zu „Burn-out“ wurden Texte der Webportale von „NetDoktor“ und „Onmeda“ ins Untersuchungskorpus aufgenommen. NetDoktor bezeichnet sich selbst als „Gesundheitsportal“, das „leicht verständliche Informationen zu Krankheiten, Symptomen, Medikamenten, Behandlungsmethoden und Laborwerten“ bereitstellt. ${ }^{383}$ Seit August 2019 gehört NetDoktor zur Hubert Burda Media, zuvor war es über mehrere Jahre Teil der Verlagsgruppe Georg von Holtzbrinck.“384 Das Portal Onmeda ist Teil der „gofeminin.de GmbH“. Beide Portale finanzieren sich nach eigenen Angaben u.a. über Werbung und Lizenzierungsartikel, die Redaktionen bestehen bei NetDoktor nach Webseitenangaben „aus Medizinern, Biologen und Fachjournalisten“" ${ }^{385}$ und bei Onmeda „aus erfahrenen Fachredakteuren und Medizinjournalisten“386. Die Adressatinnen und Adressaten sind prinzipiell alle an Gesundheitsthemen interessierten Personen.

Die Texte auf NetDoktor und Onmeda $\mathrm{zu}>$ BURNOUT « weisen in den Fließtexten weniger Verlinkungen $\mathrm{zu}$ anderen Unterseiten der Plattform auf als auf Wikipedia. Es werden aber ebenfalls Stichwörter verlinkt, die zu anderen Unterseiten der Plattformen führen (z. B. Stichwörter wie „Stressauslöser“, „Schlafstörungen“, „Depression“, „Angst“, „Verhaltenstherapie“ etc.). Ein verlinktes Inhaltsverzeichnis zu Beginn ermöglicht das Springen innerhalb der Artikel. ${ }^{387}$ Der Fachlichkeitsgrad ist gemessen an der Dichte der (erläuterten) Termini niedriger als im Wikipedia-Eintrag. Die NetDoktor- und OnmedaSeiten weisen jedoch mit eingebundenen Informationsvideos zum Beispiel zum Thema „Was ist Stress?“ und Bildergalerien wie „Burnout - die besten Tipps

383 Siehe unter: https://www.netdoktor.de/ueber-uns/ (zuletzt eingesehen am 3.12.2019).

384 Diese Informationen sind abrufbar unter: http://www.netdoktor.de/ueber-uns/, (eingesehen am 20.4.2017 und zuletzt am 03.12.2019) sowie https://www.horizont.net/medien/nachrichten/-Holtzbrinck-geht-im-Netz-auf-Shoppingtour-70907 und https://meedia.de/2019/08/05/ holtzbrinck-gibt-netdoktor-de-ab-burda-uebernimmt-online-gesundheitsportal/, zuletzt eingesehen am 03.12.2019.

$385 \mathrm{https} / / /$ www.netdoktor.de/ueber-uns/ (zuletzt eingesehen am 3.12.2019).

386 https://www.onmeda.de/ueber_uns-qualitaetsrichtlinien-4209-3.html (zuletzt eingesehen am 03.12.2019).

387 Es wurden auf NetDoktor und Onmeda die aktuellsten Artikelversionen zu „Burnout“ abgerufen. Einsehbar unter https://www.netdoktor.de/krankheiten/burnout/ (eine Artikelversion vom 28.6.2018= Dobmeier/Fux 2018) und https://www.onmeda.de/krankheiten/burnout_syndrom.html (eine Artikelversion vom 6.9.2019= Nagel 2019), (beide Links zuletzt eingesehen am 3.12.2019). Des Weiteren wurde jeweils eine ältere Artikelversion ins Korpus aufgenommen. Die ältere Artikelversion bei NetDoktor wurde am 15.6.2016 unter http://www.netdoktor.de/krankheiten/burnout/praevention/ abgerufen (= Dobmeier 2016). Bei Onmeda stammt die ältere Version vom 17. Juli 2018 (= Nagel 2018, zuletzt eingesehen am 26.03.2019). 
gegen das Ausbrennen“ (auf NetDoktor, Dobeier/Fux 2018) oder einem Link zu einem Burnout-Test (auf Onmeda, Nagel 2019) ${ }^{388}$ und interaktiven Formaten wie einem „Symptom-Checker“, „Laborwert-Checker“ oder „Heilpflanzenfinder“ (auf NetDoktor) mehr multimediale Ressourcen als der Wikipedia-Artikel auf. Die Artikel zeigen des Weiteren einige Formulierungen, die typisch für ratgebende Aufklärungstexte sind, wie z. B. „gezielte Entspannung kann man lernen [...] probieren Sie diese einfache Übung“ (Dobmeier/Fux 2018) oder „,[B]eleuchten Sie Ihren eigenen Perfektionsdrang“ und „[L]angfristig sollten Menschen mit Burnout versuchen, bessere Strategien zur Stressbewältigung im Alltag zu entwickeln“ (Nagel 2019, zu weiteren Kriterien für ratgebende Aufklärungstexte siehe Kap. 5.3.3.4).

\subsection{Zusammenfassung}

In Kapitel 5.1 wurde die Erstellung des fachlichen und fachexternen Untersuchungskorpus (Kap. 5.1.2 und 5.1.3) und eines Wörterbuch- und Datenbankkorpus (Kap. 5.1.1), dessen Belege bis ins 15. Jahrhundert zurückgehen, begründet und dargelegt. Das letztgenannte Korpus wird in Kapitel 6.1 einer eingehenden Analyse unterzogen, um herauszufinden, wann man den Beginn des Burnoutdiskurses ansetzen kann (Kap. 6.1.1) und in welcher Weise die Ausdrücke to burn(out) und (aus)brennen schon vor dem Beginn des Burnoutdiskurses in metaphorischer Weise verwendet wurden (Kap. 6.1.2).

In Kapitel 5.2 wurden fachkulturelle, sozialpolitische und gesellschaftliche Entwicklungen zusammengefasst, die sich bei der Recherche zur Zusammenstellung der Diskurskorpora zum Phänomen/Begriff `BURNOUT` als wichtige Rahmenbedingungen des Diskurses erwiesen haben, wie zum Beispiel die Entwicklung des Fachs der „Arbeits- und Organisationspsychologie“ oder das in den 1970er Jahren bundespolitisch geförderte Programm „Forschung zur Humanisierung des Arbeitslebens“ (siehe Kap. 5.2).

In Kapitel 5.3 wurden schließlich die Texte des fachlichen und fachexternen Diskursstrangs aus einer varietäten- und soziolinguistischen Perspektive und hinsichtlich fach- und vermittlungskommunikativer sowie textlinguistischer bzw. -sortenspezifischer Aspekte charakterisiert. Dadurch wurde ein Analysegerüst „,vom Standpunkt der Gesamtsprache“ (Felder 2016: 43) und vom Gesamtkorpus aus geschaffen, das im folgenden Großkapitel 6 genutzt werden

388 Abrufbar unter: https://www.onmeda.de/selbsttests/burnout_test.html (= Onmeda/Jaggi 2019, zuletzt eingesehen am 3.12.2019). 
kann, um zu vergleichen, ob sich diese verschiedenen textuellen Erscheinungsformen des fachlichen und öffentlichen Diskursstrangs in der Wahl der sprachlichen Mittel und Praktiken des Definierens ähneln oder voneinander unterscheiden. 